In cooperation with the San Antonio Water System

Geodatabase Design and Characteristics of Geologic Information for a Geodatabase of Selected Wells Penetrating the Austin Group in Central Bexar County, Texas, 2010

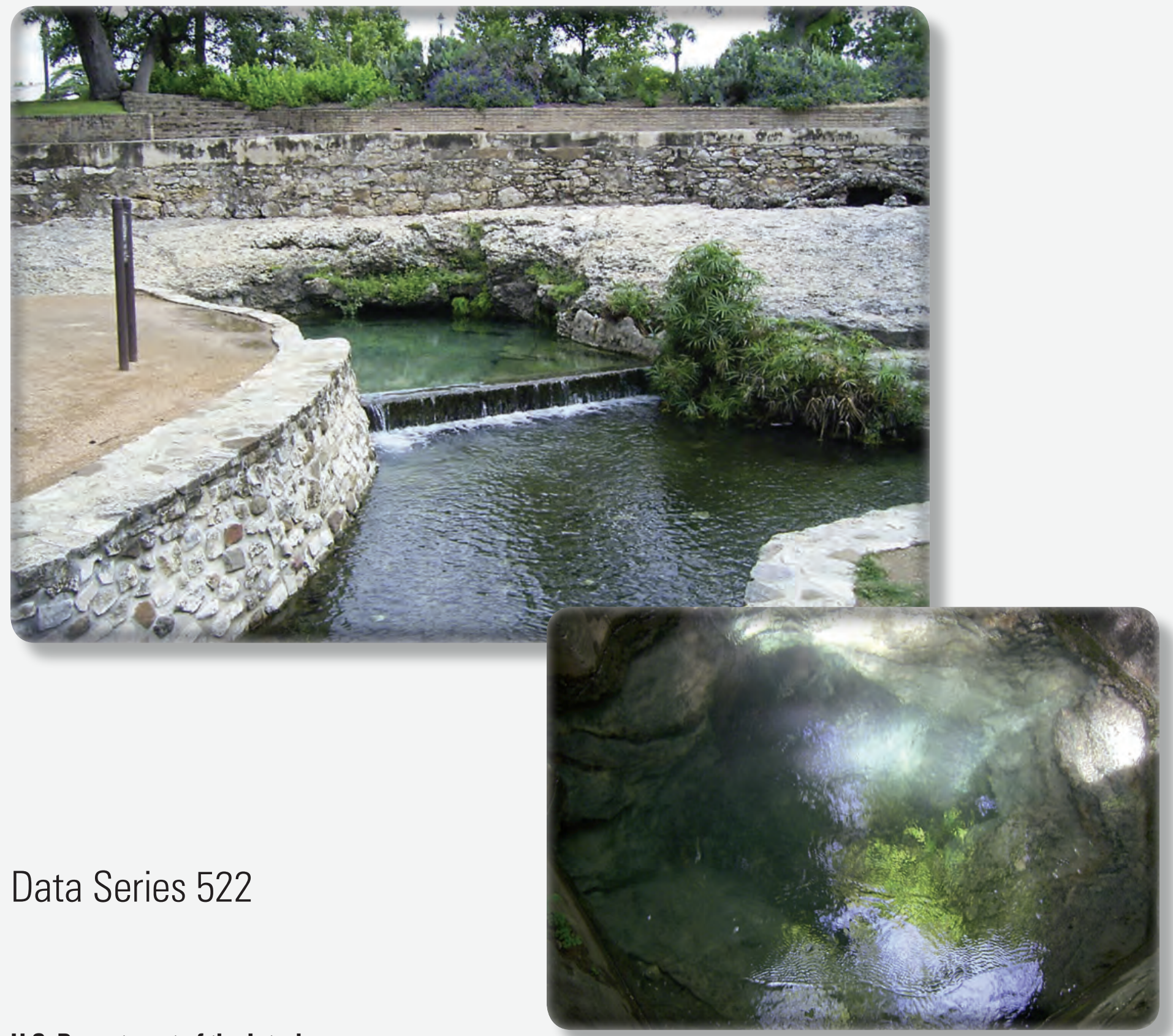


Front cover:

Top, San Pedro Springs, Bexar County, Texas, 2007 (photograph by Allan Clark, U.S. Geological Survey).

Bottom, Blue Hole (San Antonio Springs), San Antonio, Texas, 2007 (photograph by Allan Clark, U.S. Geological Survey). 


\section{Geodatabase Design and Characteristics of Geologic Information for a Geodatabase of Selected Wells Penetrating the Austin Group in Central Bexar County, Texas, 2010}

By Diana E. Pedraza and Sachin D. Shah

In cooperation with the San Antonio Water System

Data Series 522 


\section{U.S. Department of the Interior \\ KEN SALAZAR, Secretary \\ U.S. Geological Survey \\ Marcia K. McNutt, Director}

U.S. Geological Survey, Reston, Virginia: 2010

This and other USGS information products are available at http://store.usgs.gov/
U.S. Geological Survey
Box 25286 , Denver Federal Center
Denver, CO 80225
To learn about the USGS and its information products visit http://www.usgs.gov/
1-888-ASK-USGS

Any use of trade, product, or firm names is for descriptive purposes only and does not imply endorsement by the U.S. Government.

Although this report is in the public domain, permission must be secured from the individual copyright owners to reproduce any copyrighted materials contained within this report.

Suggested citation:

Pedraza, D.E., and Shah, S.D., 2010, Geodatabase design and characteristics of geologic information for a geodatabase of selected wells penetrating the Austin Group in central Bexar County, Texas, 2010: U.S. Geological Survey Data Series 522, 39 p. 


\section{Acknowledgments}

The authors thank Chris Villarreal, U.S. Environmental Protection Agency, for providing access and well-construction information for recently drilled wells in central Bexar County,

Texas. The authors also thank Jesse Mireles, Edwards Aquifer Authority, and Phil Pearce, Pape Dawson, Inc., for providing data for the Austin Group in Bexar County. 
Blank Page 


\section{Contents}

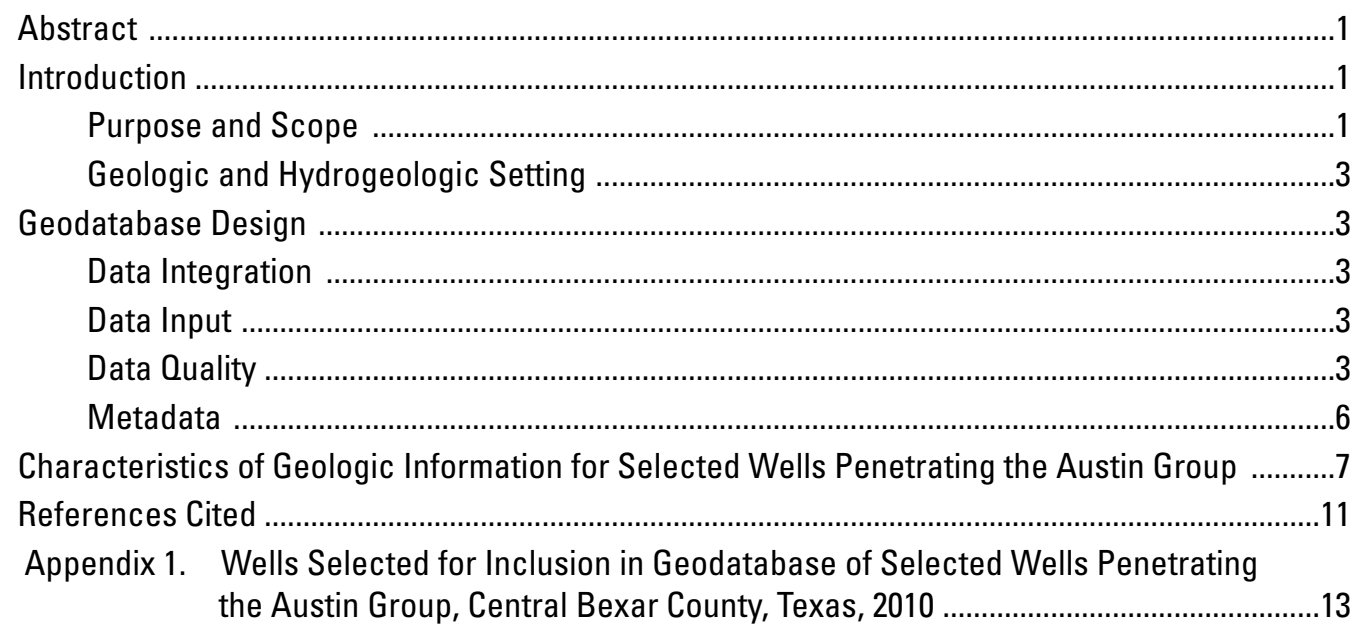

\section{Figures}

1-5. Maps showing:

1. Location of study area and selected wells penetrating the Austin Group in central Bexar County, Texas, 2010

2. Well-numbering system for the Texas Water Development Board GroundWater Data System

3. Surficial geologic units and locations of selected wells penetrating the Austin Group in central Bexar County, Texas, 2010

4. Thickness of the Austin Group for selected wells in central Bexar County, Texas, 2010

5. Depths of selected wells penetrating the Austin Group in central Bexar County, Texas, 2010

\section{Tables}

1. Summary of lithologic and hydrologic properties of stratigraphic units in central Bexar County, Texas, 2010

2. Description and definition of data compiled and entered into geodatabase of geologic information for selected wells penetrating the Austin Group in central Bexar County, Texas, 2010

3. Number and percentage of selected wells penetrating the Austin Group, categorized by surficial geology, in central Bexar County, Texas, 2010 


\section{Conversion Factors and Datums}

\section{Inch/Pound to SI}

\begin{tabular}{|c|c|c|}
\hline Multiply & By & To obtain \\
\hline \multicolumn{3}{|c|}{ Length } \\
\hline foot (ft) & 0.3048 & meter $(\mathrm{m})$ \\
\hline mile (mi) & 1.609 & kilometer $(\mathrm{km})$ \\
\hline \multicolumn{3}{|c|}{ Area } \\
\hline square mile $\left(\mathrm{mi}^{2}\right)$ & 2.590 & square kilometer $\left(\mathrm{km}^{2}\right)$ \\
\hline
\end{tabular}

\section{Datums}

Vertical coordinate information is referenced to the North American Vertical Datum of 1988 (NAVD 88).

Horizontal coordinate information is referenced to the North American Datum of 1983 (NAD 83).

Altitude, as used in this report, refers to distance above the vertical datum. 


\title{
Geodatabase Design and Characteristics of Geologic Information for a Geodatabase of Selected Wells Penetrating the Austin Group in Central Bexar County, Texas, 2010
}

\author{
By Diana E. Pedraza and Sachin D. Shah
}

\section{Abstract}

The U.S. Geological Survey, in cooperation with the San Antonio Water System, developed a geodatabase of geologic and hydrogeologic information for selected wells penetrating the Austin Group in central Bexar County, Texas. The Austin Group functions as an upper confining unit to the Edwards aquifer and is the thickest and most permeable of the Edwards aquifer confining units. The geologic and hydrogeologic information pertains to a 377-square-mile study area that encompasses central Bexar County. Data were compiled primarily from drillers' and borehole geophysical logs from Federal, State, and local agencies and published reports. Austin Group characteristics compiled for 523 unique wells are documented (if known), including year drilled, well depth, altitude of top and base of the Austin Group, and thickness of the Austin Group.

\section{Introduction}

The Austin Group in central Bexar County, Tex. (fig. 1), overlies the Eagle Ford Group and consists of chalky, variably marly, generally fossiliferous limestone, commonly containing the fossil oyster Gryphaea aucella (Small, 1986; Hanson and Small, 1995). The Austin Group functions as an upper confining unit to the Edwards aquifer or as an aquifer where it is connected to the Edwards aquifer by faults, fractures, or caves; it is the thickest and most permeable of the Edwards aquifer confining units (Small, 1986; Clark, 2003). The available geologic and hydrogeologic information on the Austin Group in Bexar County is sparse. As demand for water increases in the San Antonio area, all available water resources will need to be investigated and characterized. There is a need by waterresource managers to gain a better understanding of the waterbearing and chemical characteristics of the Austin Group in Bexar County, Tex.

Much of the available information regarding the waterbearing and chemical characteristics of the Austin Group is in well logs produced when the wells were initially drilled; some of the information has been published by various entities but never compiled into one geodatabase. Some of the information is stored by various agencies and is available upon request. A first step toward achieving a better understanding of the hydrologic properties of the Austin Group was to compile existing geologic and hydrogeologic data and to organize that information in a geodatabase.

The U.S. Geological Survey (USGS), in cooperation with the San Antonio Water System (SAWS), developed a geodatabase (also referred to as a geospatial database) of geologic and hydrogeologic information from wells penetrating the Austin Group. Existing geologic and hydrogeologic information pertaining to 523 unique wells in a 377-square-mile study area (fig. 1) encompassing central Bexar County was compiled. The information, in geodatabase format (Zeiler, 1999), includes the following data measured or recorded at each well (if known): year drilled, well depth, altitude of top and base of the Austin Group, thickness of the Austin Group, and source of the data. The geodatabase is limited to selected digital and hard-copy data available from various Federal, State, or local agencies including the U.S. Environmental Protection Agency (USEPA), Texas Water Development Board (TWDB), Edwards Aquifer Authority (EAA), and SAWS; and from published reports.

\section{Purpose and Scope}

The purpose of this report is to document the design and integration of geologic and hydrogeologic information for selected wells penetrating the Austin Group in a 377-square-mile study area in central Bexar County, Tex., into a geodatabase. The design and integration of geologic and hydrogeologic information into a series of relational tables in the geodatabase are described. Austin Group characteristics compiled at 523 unique wells are documented (if known), including year drilled, well depth, altitude of top and base of the Austin Group, and thickness of the Austin Group. The different sources used to populate the geodatabase are documented and the methodology used to incorporate the 


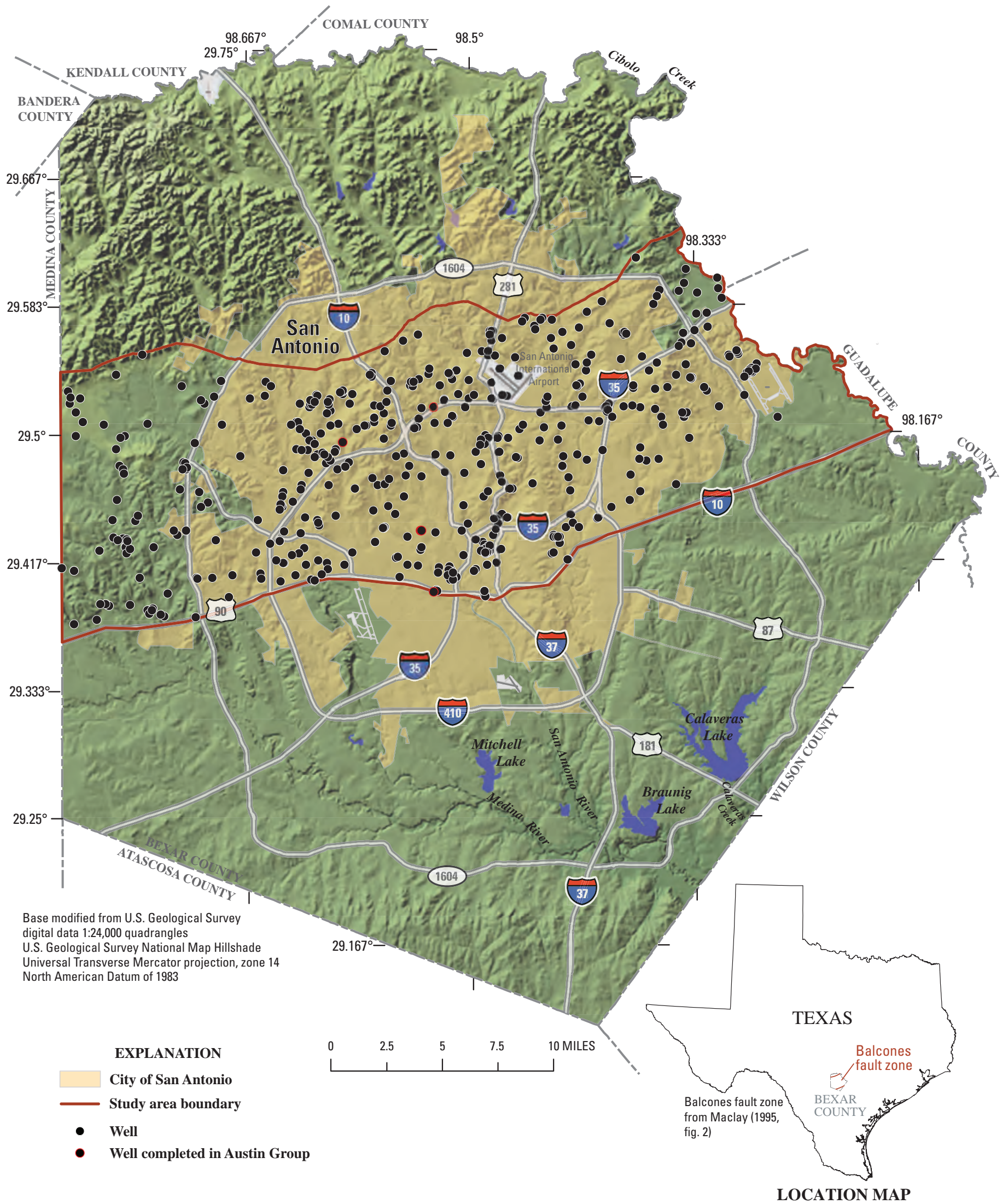

Figure 1. Location of study area and selected wells penetrating the Austin Group in central Bexar County, Texas, 2010. 
geologic information in the geodatabase is described. Existing digital and hard-copy data were entered into the geodatabase; no new data were collected.

\section{Geologic and Hydrogeologic Setting}

The Austin Group is part of a series of geologic formations overlying the Edwards aquifer (Small, 1986); informally the Austin Group is referred to as the Austin Chalk. It is the thickest unit of the upper confining units of the Edwards aquifer (table 1). Arnow (1963) noted that the Austin Group is subject to structural controls associated with the Balcones fault zone. In addition to structural controls, the carbonate rock layers are subject to localized groundwater flow and associated limestone dissolution that might enhance the permeability of fault and fracture systems, including solution enlargement at a scale ranging from individual fractures to cave networks (Ferrill and others, 2004).

Recharge entering the Austin Group in Bexar County is derived from multiple sources, including direct infiltration of precipitation, infiltration along stream channels, and water moving up into the Austin Group from the underlying Edwards aquifer (Arnow, 1963). Because the connection between the land surface and the Austin Group is enhanced by karst features, infiltration is rapid and little opportunity exists for any attenuation of contaminants by geochemical or biological processes (Stephenson and others, 1999).

\section{Geodatabase Design}

A geodatabase is a spatially enabled database that contains both spatial and tabular data and allows users to associate tabular data with physical and spatial components (Shah and Houston, 2007). Geographically referenced data from a geodatabase can be manipulated using a geographic information system (GIS) to produce maps, interactive queries, and various types of spatial analyses. A geodatabase provides a framework and an interactive tool to aid in understanding attributes of geologic formations such as subsurface structure. The geodatabase designed for this study was based on an ESRI ArcGIS personal geodatabase platform. ArcGIS personal geodatabases were stored as Microsoft Access files (Zeiler, 1999).

A geodatabase uses a thematic approach to create spatial layers of data, called feature classes, in a GIS. Feature classes represent the various types of point, line, and polygon data that are keyed to spatial location and are related to one another within the geodatabase. The various types of data are separated into relational tables in the geodatabase (table 2) on the basis of how these data interact and correspond with the spatial feature class. These relational tables represent a collection of features and the relations between them (Shah and Houston, 2007). The goal is to provide accurate representations of the spatial extent and properties of the Austin Group using the geologic and hydrogeologic data that have been compiled. Compiling data, entering data into the geodatabase, ensuring data quality, and documenting the associated metadata were the primary steps in creating the geodatabase.

\section{Data Integration}

Subsurface geologic data were organized and incorporated into the geodatabase. Data were compiled primarily from drillers' and borehole geophysical logs from Federal, State, and local government agencies and from maps in published reports. Drillers' and geophysical logs were used to obtain lithology and altitude of the top and base of the Austin Group. Data gaps exist in parts of the study area; for example, at some sites drillers did not describe the lithology and thickness of the Austin Group as separate and distinct from the underlying unit where the two units showed similar lithologic characteristics, thus precluding identification of the base of the Austin Group at those wells.

\section{Data Input}

Digital data were imported and hard-copy data were entered manually into the geodatabase. Because the scope of geodatabase development was limited to geologic and hydrogeologic data, only the Well_Locations point feature class and wells and lithology tables containing geologic and hydrogeologic attributes were populated. Selected readily available spatial data from drillers' and geophysical logs were input into the Well_Locations point feature class. Related well and lithologic data were populated in corresponding tables. Additional features classes were included in the geodatabase as supporting geospatial data. Examples of such feature classes are GAT_faults, GAT_surface_geology, Counties, Study_Area, and Parks. A complete listing and description of the feature classes and tables that compose the geodatabase are in table 2. Drillers' logs of wells were recorded at the time of drilling. Wells were subsequently assigned a State well number (fig. 2) using the TWDB GroundWater Data System (GWDS) (Nordstrom and Quincy, 1999). Wells not in the GWDS were assigned two arbitrary characters to distinguish well locations from one another. The key well number, the first five digits, retains the location aspect of a State well number - that is, wells sited in increasingly smaller quadrangles.

\section{Data Quality}

Quality-assurance techniques were applied to ensure the quality of the data entered into the geodatabase. Queries were done on 755 well entries in the geodatabase to find missing records, duplicate records, incomplete well records, and values that were internally inconsistent for a given well (for example, altitude of the top or bottom of the Austin Group exceeding the reported well depth or erroneous recorded thickness for the Austin Group). When duplicate well records 


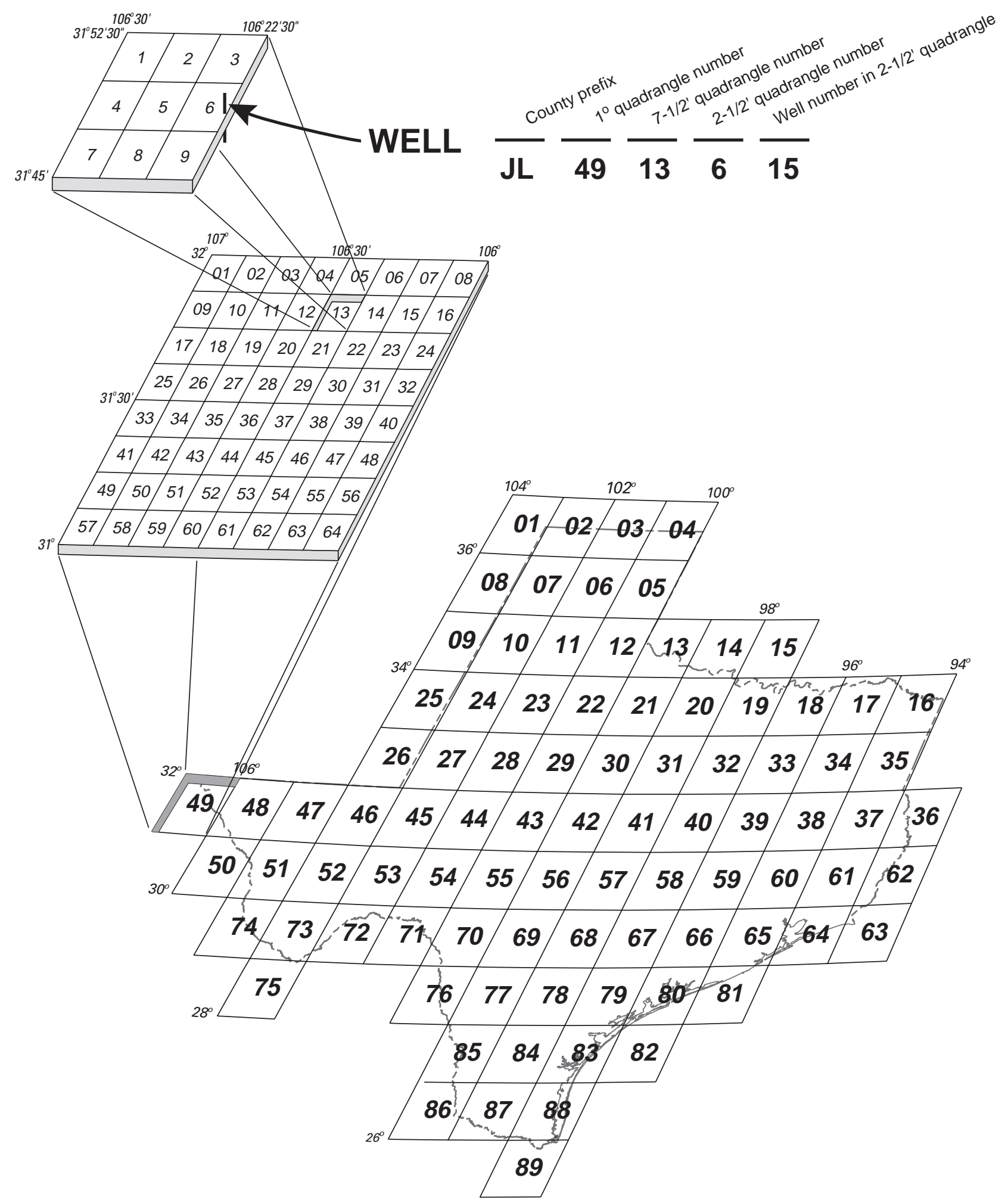

Figure 2. Well-numbering system for the Texas Water Development Board GroundWater Data System (modified from Nordstrom and Quincy, 1999). 
Table 1. Summary of lithologic and hydrologic properties of stratigraphic units in central Bexar County, Texas, 2010.

[Hydrogeologic units modified from Maclay (1995); groups and formations modified from Sellards and others (1923) and Rose (1972); lithology modified from Dunham (1962); thickness modified from Livingston (1947), Stein and Ozuna (1995), and Blome and others (2005); water-yielding characteristics modified from Livingston (1947). AQ, aquifer; --, not available; CU, confining unit]

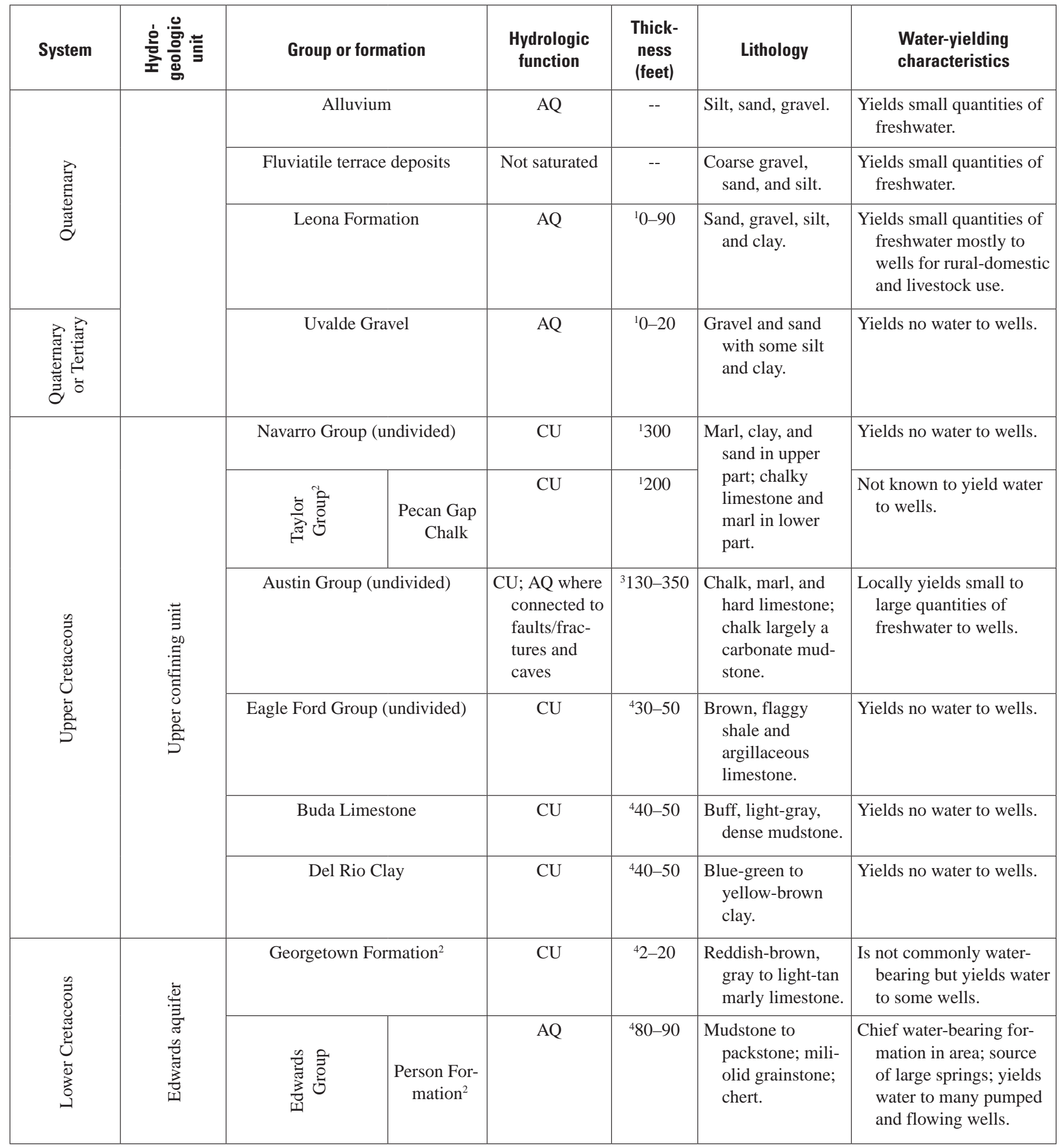

${ }^{1}$ Livingston, 1947.

${ }^{2}$ Not shown in study area.

${ }^{3}$ Stein and Ozuna, 1995.

${ }^{4}$ Blome and others, 2005 
Table 2. Description and definition of data compiled and entered into geodatabase of geologic information for selected wells penetrating the Austin Group in central Bexar County, Texas, 2010.

[GAT, Geologic Atlas of Texas; --, not applicable]

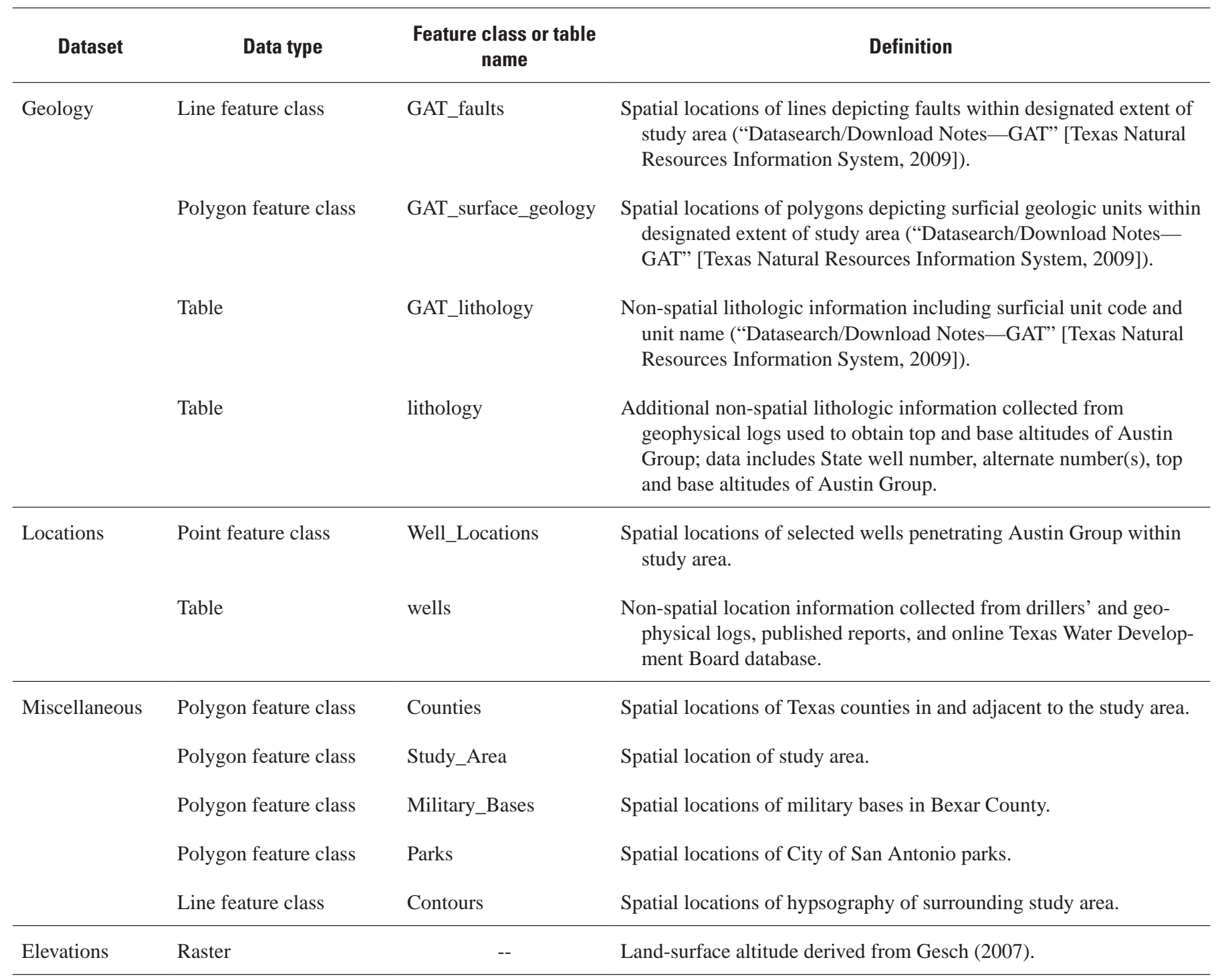

were identified, database queries and GIS proximity analyses were used to combine duplicate records into a single well record. Three duplicate well records with different altitudes for the top and bottom of the Austin Group, obtained from different published sources, were kept in the database because it was not possible to determine which of the two records for each of these wells contained the correct information. Duplicate records were kept for wells AY-68-30-616a and AY-68-30-616b, AY-68-36-610a and AY-68-36-610b, and AY-68-37-203a and AY-68-37-203b. After verification that well records were correct, 112 other duplicate well records and 117 incomplete well records were removed, and 526 records representing 523 unique wells with geologic data were included in the final geodatabase. All wells penetrating the Austin Group that were compiled in the geodatabase and their respective locations are listed in appendix 1 .

\section{Metadata}

Metadata that comply with Federal Geographic Data Committee (2009) standards were created for each spatial component. The metadata record documents the basic characteristics of the data or information resource in the study area. Metadata components include source document information such as title, abstract, and publication date; geographic elements such as geographic extent and projection information; and database elements such as attribute label definitions and attribute domain values. 


\section{Characteristics of Geologic Information for Selected Wells Penetrating the Austin Group}

Summaries of the geologic and descriptive well information input to the geodatabase are shown in figures 3-5. Each figure shows the distribution of wells penetrating the Austin Group relative to various geologic or spatial attributes, including surface geology (fig. 3; table 3), range in thickness of the Austin Group in each well (fig. 4), and range in depth of wells, whether completed in the Austin Group or in a deeper formation (fig. 5). Appendix 1 lists all wells penetrating the Austin Group included in the geodatabase and geologic information regarding the Austin Group obtained during the installation of a given well or summarized in reports discussing selected wells.

Figure 3 shows the surficial geology of wells throughout the study area. The highest percentage of wells plot where the surface geology is the Pecan Gap Chalk (table 3). The next highest percentage of wells plot where the surface geology is the fluviatile terrace deposits. No wells plot where the surface geology is the Eagle Ford Group or the Del Rio Clay (formations that underlie the Austin Group).

Distribution of wells categorized by the thickness of the Austin Group where the well was drilled is shown in figure 4. For wells with known depths in the geodatabase, thickness of the Austin Group ranges from 8 to 388 feet, and the average thickness is about 150 feet. The thickness of the Austin Group ranges from 80 to 160 feet for 357 wells and exceeds 300 feet for 10 wells.

Five wells in the study area are known to have been completed only in the Austin Group (indicated in figs. 3-5 by a red circle around the well symbol). All other wells are completed in the Edwards Limestone.

The distribution of wells by well depth, in feet below land-surface datum (LSD), for the study area is shown on figure 5. Depths for the selected wells range from 120 to 1,652 feet below LSD. The depths of more than one-half the wells range from 301 to 900 feet below LSD. The average well depth is about 715 feet below LSD. Less than 3 percent of all wells have depths greater than 1,300 feet below LSD. 


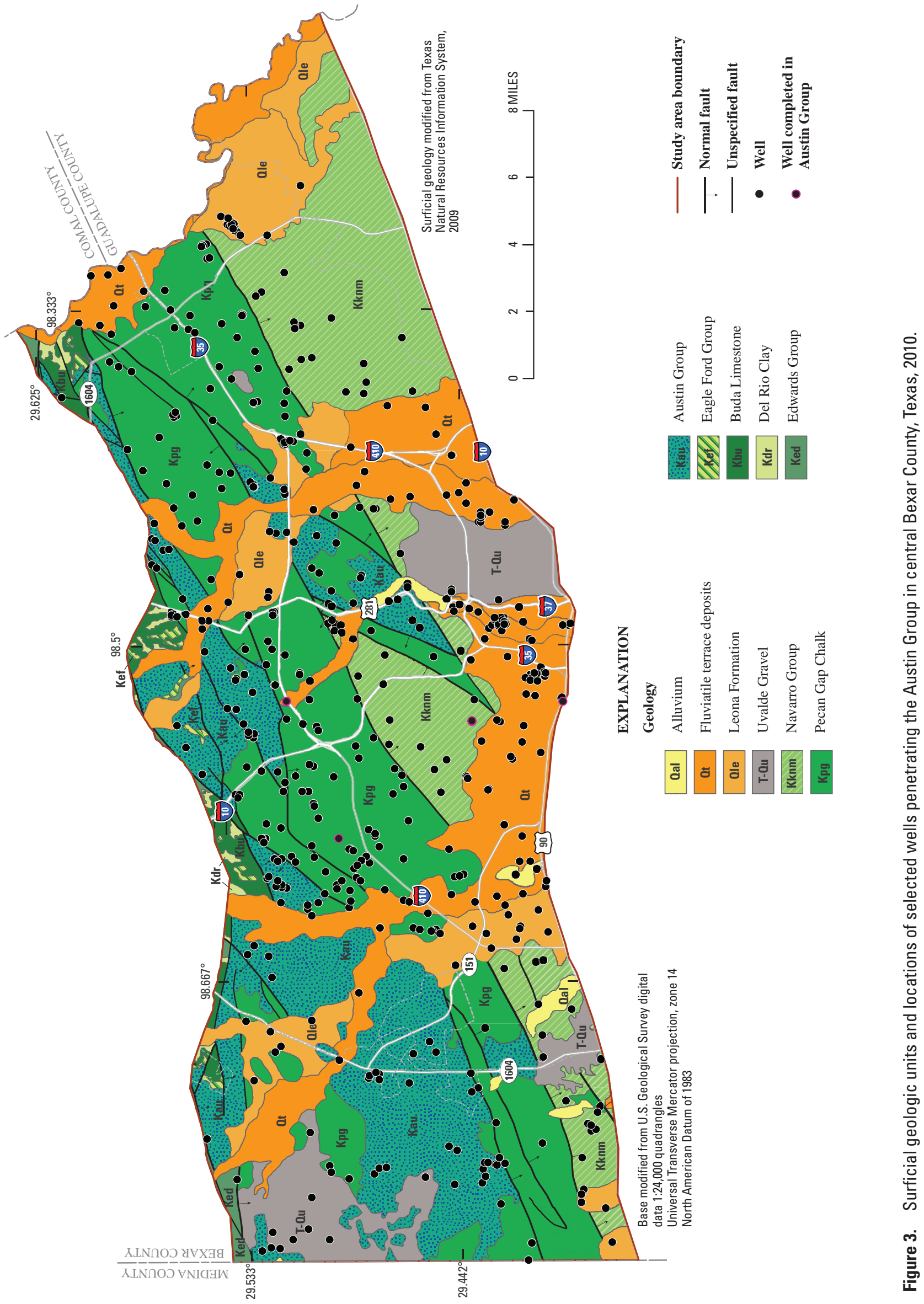




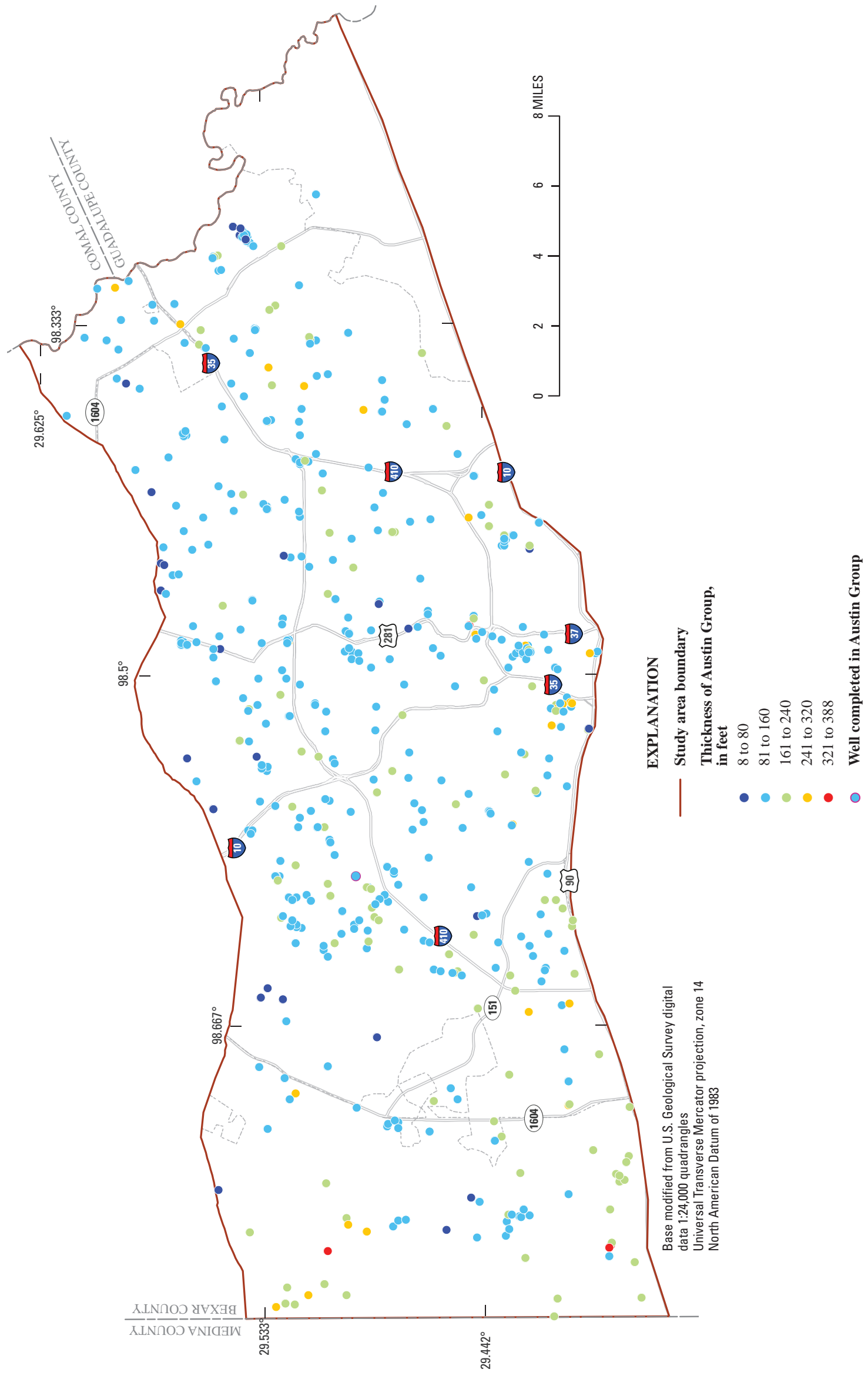




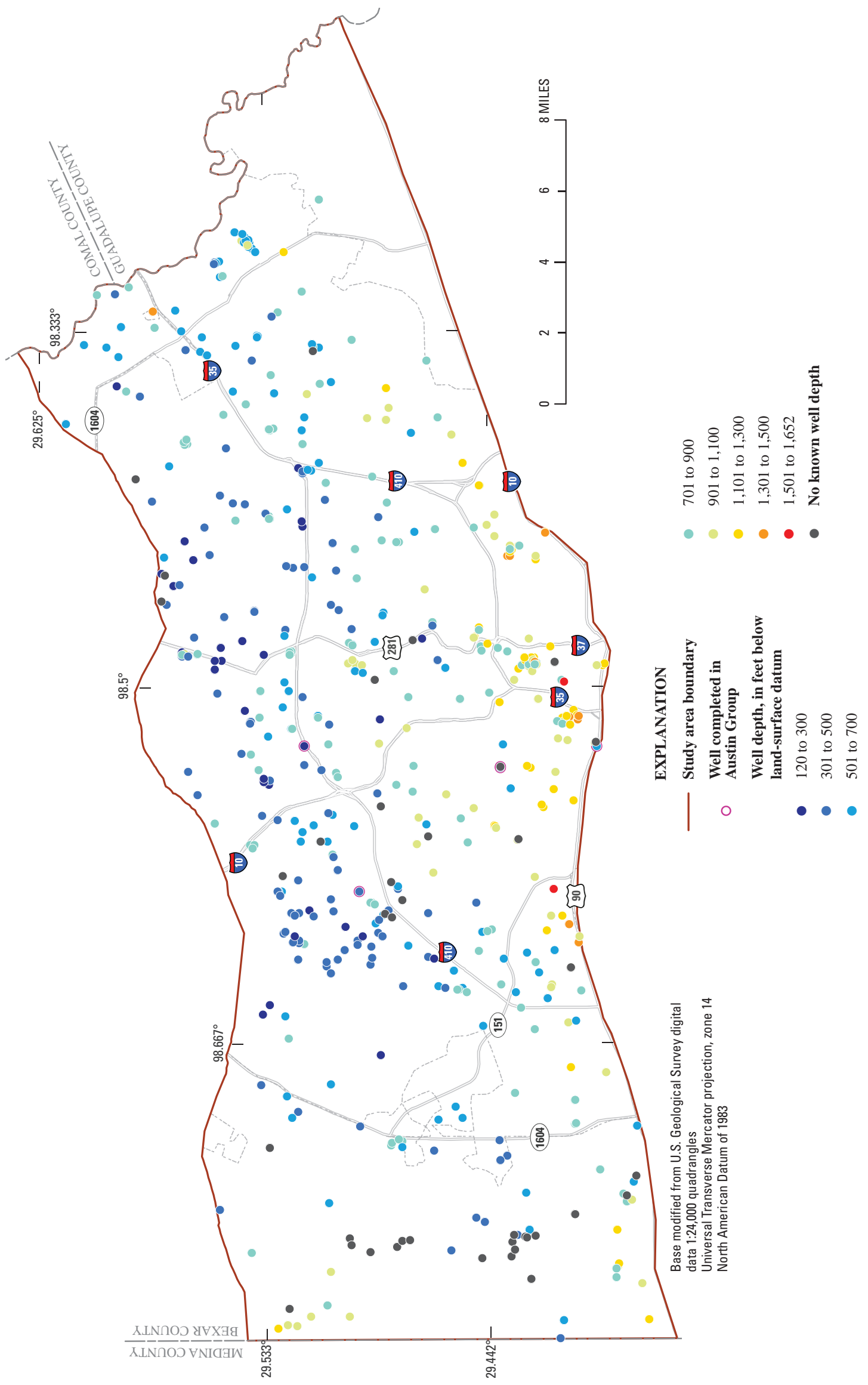

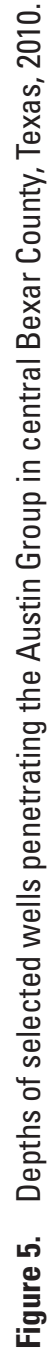


Table 3. Number and percentage of selected wells penetrating the Austin Group, categorized by surficial geology, in central Bexar County, Texas, 2010.

\begin{tabular}{clcc}
\hline $\begin{array}{c}\text { Geologic } \\
\text { abbreviation } \\
\text { (fig. 3) }\end{array}$ & \multicolumn{1}{c}{$\begin{array}{c}\text { Surficial } \\
\text { geologic unit }\end{array}$} & $\begin{array}{c}\text { Number } \\
\text { of wells }\end{array}$ & $\begin{array}{c}\text { Percent- } \\
\text { age } \\
\text { of wells }\end{array}$ \\
\hline Qal & Alluvium & 5 & 0.96 \\
Qt & Fluviatile terrace deposits & 133 & 25.43 \\
Qle & Leona Formation & 41 & 7.84 \\
T-Qu & Uvalde Gravel & 14 & 2.68 \\
Kknm & Navarro Group & 54 & 10.33 \\
Kpg & Pecan Gap Chalk & 187 & 35.76 \\
Kau & Austin Group & 84 & 16.06 \\
Kbu & Buda Limestone & 4 & .76 \\
Ked & Edwards Group & 1 & .19 \\
& & & \\
& & 523 & 100 \\
\hline
\end{tabular}

\section{References Cited}

Arnow, Ted, 1963, Ground-water geology of Bexar County, Texas: U.S. Geological Survey Water-Supply Paper 1588, $36 \mathrm{p}$.

Blome, C.D., Faith, J.R., Pedraza, D.E., Ozuna, G.B., Cole, J.C., Clark, A.K., Small, T.A., and Morris, R.R., comps., 2005, Geologic map of the Edwards aquifer recharge zone, south-central Texas: U.S. Geological Survey Scientific Investigations Map 2873, version 1.1, 1 pl., scale 1:200,000.

Clark, A.K., 2003, Geologic framework and hydrogeologic characteristics of the Edwards aquifer, Uvalde County, Texas: U.S. Geological Survey Water-Resources Investigations Report 03-4010, 17 p., 1 pl., accessed May 4, 2010 at http://pubs.er.usgs.gov/usgspubs/wri/wri034010.

Dunham, R.J., 1962, Classification of carbonate rocks according to depositional texture, in Classification of Carbonate Rocks Symposium: American Association of Petroleum Geologists Memoir 1, p. 108-121.

Federal Geographic Data Committee, 2009, Metadata: accessed December 2009 at $h t t p: / / w w w . f g d c . g o v / i n d e x$. html.

Ferrill, D.A., Sims, D.W., Waiting, D.J., Morris, A.P., Franklin, N., and Schultz, A.L., 2004, Structural framework of the Edwards aquifer recharge zone, south-central Texas: Geological Society of America Bulletin, v. 116, 12 p.
Gesch, D.B., 2007, The National Elevation Dataset, in Maune, D., ed., Digital elevation model technologies and applications-The DEM users manual ( $2 \mathrm{~d}$ ed.): Bethesda, Md., American Society for Photogrammetry and Remote Sensing, p. 99-118.

Hanson, J.A., and Small, T.A., 1995, Geologic framework and hydrogeologic characteristics of the Edwards aquifer outcrop, Hays County, Texas: U.S. Geological Survey WaterResources Investigations Report 95-4265, $10 \mathrm{p}$.

Livingston, Penn, 1947, Ground-water resources of Bexar County, Texas: Texas Board of Water Engineers Miscellaneous Publication M-13, $235 \mathrm{p}$.

Maclay, R.W., 1995, Geology and hydrology of the Edwards aquifer in the San Antonio area, Texas: U.S. Geological Survey Water-Resources Investigations Report 95-4186, $64 \mathrm{p}$.

Nordstrom, P.L., and Quincy, Roger, 1999, GroundWater Data System data dictionary: Texas Water Development Board User Manual 50, 99 p.

Petitt, B.M., Jr., and George, W.O., 1956, Ground-water resources of the San Antonio area, Texas-Records of wells and springs: Texas Board of Water Engineers Bulletin 5608, v. II, part I, 252 p.

Rose, P.R., 1972, Edwards Group, surface and subsurface, central Texas: Austin, University of Texas, Bureau of Economic Geology Report of Investigations 74, 198 p.

Sellards, E.H., Adkins, W.S., and Plummer, F.B., 1923, The geology of Texas-Volume I, Stratigraphy: Austin, University of Texas, Bureau of Economic Geology Bulletin 3232, $1,007 \mathrm{p}$.

Shah, S.D., and Houston, N.A., 2007, Geologic and hydrogeologic information for a geodatabase for the Brazos River alluvium aquifer, Bosque County to Fort Bend, Texas (version 3): U.S. Geological Survey Open-File Report 2007-1031, 10 p.

Small, T.A., 1984, Identification and tabulation of geological contacts in the Edwards aquifer, San Antonio area, Texas: U.S. Geological Survey Open-File Report 84-75, 54 p.

Small, T.A., 1986, Hydrogeologic sections of the Edwards aquifer and its confining units in the San Antonio area, Texas: U.S. Geological Survey Water-Resources Investigations Report 85-4259, $52 \mathrm{p}$.

Stein, W.G., and Ozuna, G.B., 1995, Geologic framework and hydrogeologic characteristics of the Edwards aquifer recharge zone, Bexar County, Texas: U.S. Geological Survey Water-Resources Investigations Report 95-4030, 8 p., 1 plate.

Stephenson, J.B., Zhou, W.F., Beck, B.F., and Green, T.S., 1997, Highway stormwater runoff in karst areas- 
Preliminary results of baseline monitoring and design of a treatment system for a sinkhole in Knoxville, Tennessee: Engineering Geology, v. 52, p. 51-59.

Texas Natural Resources Information System, 2009, Data search/download notes-GAT, Geologic atlas of Texas: accessed May 2009 at http://www.tnris.state.tx.us/ datadownload/download.jsp.
Texas Water Development Board, 2009, Water information integration and dissemination (WIID): accessed June 4, 2009 at http://wiid.twdb.state.tx.us/index_explain. asp.

Zeiler, Michael, 1999, Modeling our world-The ESRI guide to geodatabase design: Redlands, Calif., Environmental Systems Research Institute Press, 10 p. 


\section{Appendix 1-Wells Selected for Inclusion in Geodatabase of Selected Wells Penetrating the Austin Group, Central Bexar County, Texas, 2010}


Appendix 1. Wells selected for inclusion in geodatabase of selected wells penetrating the Austin Group, central Bexar County, Texas,

[dd, decimal degrees; NAVD 88, North American Vertical Datum of 1988; --, not available; BMWD, Bexar Metropolitan Water District; USGS, U.S. Geological

\begin{tabular}{|c|c|c|c|c|c|c|c|}
\hline $\begin{array}{l}\text { State well } \\
\text { number }\end{array}$ & $\begin{array}{l}\text { First alternate } \\
\text { well identifier }\end{array}$ & $\begin{array}{l}\text { Second } \\
\text { alternate } \\
\text { well } \\
\text { identifier }\end{array}$ & $\begin{array}{c}\text { Third } \\
\text { alternate } \\
\text { well } \\
\text { identifier }\end{array}$ & $\begin{array}{l}\text { Latitude } \\
\text { (dd) }\end{array}$ & $\begin{array}{l}\text { Longitude } \\
\text { (dd) }\end{array}$ & $\begin{array}{l}\text { Land-surface } \\
\text { altitude } \\
\text { (feet above } \\
\text { NAVD 88) }\end{array}$ & $\begin{array}{l}\text { Year } \\
\text { drilled }\end{array}$ \\
\hline AY-68-26-803 & Lackland Terrace & -- & -- & 29.528562808 & 98.8000253742 & 1,045 & 1971 \\
\hline AY-68-26-804 & Sanders & D-1 & -- & 29.5246743941 & 98.7983588518 & 1,044 & 1933 \\
\hline AY-68-26-904 & Country Oaks no. 1 & -- & -- & 29.5071745804 & 98.7736354237 & 995 & 1984 \\
\hline AY-68-26-905 & BMWD no. 1 & 097 WP no. 1 & -- & 29.539722 & 98.764444 & 1,028 & 1998 \\
\hline AY-68-26-9aа & 38525 & -- & -- & 29.524167 & 98.790833 & 1,035 & -- \\
\hline AY-68-26-9bb & Bayer & -- & -- & 29.5085638732 & 98.7891917931 & 1,023 & -- \\
\hline AY-68-27-401 & -- & -- & -- & 29.5527287846 & 98.7444689072 & 1,035 & 1920 \\
\hline AY-68-27-802 & Tausch & -- & -- & 29.5230078591 & 98.701133616 & 927 & 1969 \\
\hline AY-68-27-803 & Munoz & -- & -- & 29.5202299595 & 98.6983565288 & 918 & 1970 \\
\hline AY-68-27-804 & Kindrick & -- & -- & 29.5068974383 & 98.6855781272 & 895 & 1954 \\
\hline AY-68-27-8jj & Zion & -- & -- & 29.5355071383 & 98.6858558729 & 935 & -- \\
\hline AY-68-27-903 & -- & -- & -- & 29.5068973298 & 98.633909859 & 840 & 1959 \\
\hline AY-68-27-906 & Concorde no. 1 & -- & -- & 29.5241187512 & 98.6641887069 & 992 & 1973 \\
\hline AY-68-27-907 & Concorde no. 2 & -- & -- & 29.5255076809 & 98.6536324532 & 922 & 1973 \\
\hline AY-68-27-910 & Van Deldon & -- & -- & 29.5202298278 & 98.6272427689 & 890 & 1982 \\
\hline AY-68-27-911 & Hallmark & -- & -- & 29.534673784 & 98.6527994867 & 910 & -- \\
\hline AY-68-27-9nn & Grady's & -- & -- & 29.5085635945 & 98.6302987797 & 850 & -- \\
\hline AY-68-28-604 & Lutteringer & -- & -- & 29.5649501009 & 98.5391857764 & 890 & 1956 \\
\hline AY-68-28-6bb & E-66 & -- & -- & 29.5430034359 & 98.5307804654 & 894 & 1945 \\
\hline AY-68-28-6cc & Harvath & -- & -- & 29.5530062511 & 98.5027958079 & 833 & -- \\
\hline AY-68-28-700a & Whitby no. 2 & -- & -- & 29.5057858644 & 98.6047425818 & 895 & -- \\
\hline AY-68-28-700b & Whitby H.P.R. no. 1 & -- & -- & 29.5230074644 & 98.6052982402 & 915 & -- \\
\hline AY-68-28-702 & Leon Valley & -- & -- & 29.5068972564 & 98.5989089999 & 840 & 1963 \\
\hline AY-68-28-706 & Balcones Water & -- & -- & 29.5177299056 & 98.6200205767 & 902 & 1972 \\
\hline AY-68-28-708 & Methodist Mission Home & -- & -- & 29.526062593 & 98.6150204975 & 900 & 1966 \\
\hline AY-68-28-7hh & $\mathrm{E}-48$ & -- & -- & 29.5273894142 & 98.5972794734 & 870 & 1945 \\
\hline AY-68-28-7ii & Sanville & -- & -- & 29.5224519541 & 98.6191874704 & 906 & -- \\
\hline AY-68-28-7kk & S.A. Alternative Housing & -- & -- & 29.5138411334 & 98.6072426851 & 847 & -- \\
\hline AY-68-28-711 & Pacific Scene & -- & -- & 29.5010638535 & 98.6230763391 & 862 & -- \\
\hline AY-68-28-7nn & 0798-LG & -- & -- & 29.5283333333 & 98.5952777778 & 890 & -- \\
\hline AY-68-28-7oo & 0594-CLG & -- & -- & 29.5255555556 & 98.6144444444 & 890 & -- \\
\hline AY-68-28-7pp & USAA & -- & -- & 29.5266666667 & 98.5955555556 & 887 & -- \\
\hline AY-68-28-7qq & Studer & -- & -- & 29.5199520437 & 98.618354103 & 915 & -- \\
\hline
\end{tabular}


2010.

Survey; LSD, land-surface datum; TWDB, Texas Water Development Board; EAA, Edwards Aquifer Authority; SAWS, San Antonio Water System]

\begin{tabular}{|c|c|c|c|c|c|c|c|c|}
\hline $\begin{array}{l}\text { State well } \\
\text { number }\end{array}$ & $\begin{array}{l}\text { Well depth } \\
\text { (feet } \\
\text { below } \\
\text { LSD) }\end{array}$ & $\begin{array}{c}\text { Altitude of } \\
\text { top of Aus- } \\
\text { tin Group } \\
\text { (feet above } \\
\text { NAVD 88) }\end{array}$ & $\begin{array}{c}\text { Altitude of } \\
\text { base of Aus- } \\
\text { tin Group } \\
\text { (feet above } \\
\text { NAVD 88) }\end{array}$ & $\begin{array}{c}\text { Thickness } \\
\text { of Austin } \\
\text { Group } \\
\text { (feet) }\end{array}$ & Data source & Source agency & $\begin{array}{c}\text { Data } \\
\text { source date }\end{array}$ & $\begin{array}{c}\text { Report } \\
\text { author, } \\
\text { year }\end{array}$ \\
\hline AY-68-26-803 & 1,140 & 620 & 349 & 271 & drillers' log & TWDB & $3 / 2 / 1971$ & TWDB, 2009 \\
\hline AY-68-26-804 & 1,000 & 462 & 282 & 180 & geophysical log & TWDB & $3 / 13 / 1978$ & Petitt and George, 1956 \\
\hline AY-68-26-805 & 1,010 & 525 & 345 & 180 & geophysical log & TWDB & $3 / 28 / 1972$ & TWDB, 2009 \\
\hline AY-68-26-806 & 997 & 607 & 366 & 241 & drillers' log & TWDB & $8 / 14 / 1981$ & TWDB, 2009 \\
\hline AY-68-26-904 & 920 & 665 & 307 & 358 & drillers' log & BMWD & $6 / 14 / 1984$ & unpublished data, 1984 \\
\hline AY-68-26-905 & 870 & 1,028 & 795 & 233 & drillers' log & BMWD & $11 / 23 / 1998$ & unpublished data, 1998 \\
\hline AY-68-26-9aa & -- & 465 & 265 & 200 & drillers' log & TWDB & $6 / 2 / 2004$ & unpublished data, 2004 \\
\hline AY-68-26-9bb & 883 & 617 & 425 & 192 & geophysical log & TWDB & $11 / 20 / 1991$ & unpublished data, 1991 \\
\hline AY-68-27-401 & 424 & 1,035 & 1,013 & 22 & drillers' log & USGS & $5 / 19 / 1972$ & unpublished data, 1972 \\
\hline AY-68-27-703 & 631 & 727 & 529 & 198 & report/publication & USGS & $5 / 31 / 1973$ & Small, 1984 \\
\hline AY-68-27-7aa & -- & 943 & 861 & 82 & report/publication & USGS & -- & Small, 1984 \\
\hline AY-68-27-801 & 518 & 792 & 668 & 124 & drillers' log & TWDB & $1 / 13 / 1968$ & TWDB, 2009 \\
\hline AY-68-27-802 & 660 & 723 & 618 & 105 & drillers' log & TWDB & $1 / 22 / 1969$ & TWDB, 2009 \\
\hline AY-68-27-803 & 420 & 918 & 663 & 255 & drillers' log & TWDB & $3 / 16 / 1970$ & TWDB, 2009 \\
\hline AY-68-27-804 & 525 & 877 & 764 & 113 & drillers' log & TWDB & $12 / 1 / 1954$ & TWDB, 2009 \\
\hline AY-68-27-8jj & 317 & 923 & 785 & 138 & geophysical log & TWDB & $8 / 31 / 1978$ & unpublished data, 1978 \\
\hline AY-68-27-903 & 470 & 809 & 665 & 144 & drillers' log & TWDB & -- & TWDB, 2009 \\
\hline AY-68-27-906 & 764 & 984 & 826 & 158 & geophysical log & USGS & $5 / 3 / 1973$ & Small, 1984 \\
\hline AY-68-27-907 & 680 & 922 & 847 & 75 & geophysical log & USGS & $8 / 13 / 1973$ & Small, 1984 \\
\hline AY-68-27-910 & 382 & 880 & 740 & 140 & drillers' log & TWDB & $11 / 30 / 1982$ & TWDB, 2009 \\
\hline AY-68-27-911 & 196 & 910 & 848 & 62 & geophysical log & TWDB & $3 / 12 / 1992$ & unpublished data, 1992 \\
\hline AY-68-27-9nn & 379 & 769 & 639 & 130 & geophysical log & TWDB & 7/14/1991 & unpublished data, 1991 \\
\hline AY-68-27-9oo & 416 & 818 & 659 & 159 & geophysical log & TWDB & 4/9/1999 & unpublished data, 1999 \\
\hline AY-68-27-9pp & 450 & 766 & 614 & 152 & geophysical log & TWDB & $1 / 28 / 1998$ & unpublished data, 1998 \\
\hline AY-68-27-9qq & 295 & 840 & 776 & 64 & geophysical log & TWDB & 6/7/1991 & unpublished data, 1991 \\
\hline AY-68-27-9rr & 391 & 852 & 676 & 176 & geophysical log & TWDB & $3 / 15 / 1989$ & unpublished data, 1989 \\
\hline AY $-68-28-500$ & 705 & 885 & 833 & 52 & geophysical log & USGS & $8 / 3 / 1988$ & unpublished data, 1988 \\
\hline AY-68-28-503 & 375 & 935 & 847 & 88 & geophysical log & USGS & $3 / 5 / 1974$ & Small, 1984 \\
\hline AY-68-28-604 & 344 & 890 & 882 & 8 & drillers' log & TWDB & $9 / 21 / 1956$ & TWDB, 2009 \\
\hline AY-68-28-6bb & 441 & 824 & 649 & 175 & report/publication & TWDB & -- & Livingston, 1947 \\
\hline AY-68-28-6cc & 325 & 833 & 731 & 102 & geophysical log & TWDB & $5 / 15 / 1989$ & unpublished data, 1989 \\
\hline AY-68-28-700a & 368 & 895 & 717 & 178 & geophysical log & EAA & $10 / 18 / 1989$ & unpublished data, 1989 \\
\hline AY-68-28-700b & 349 & 873 & 722 & 151 & geophysical log & TWDB & $10 / 18 / 1989$ & TWDB, 2009 \\
\hline AY-68-28-702 & 450 & 752 & 575 & 177 & geophysical log & EAA & $6 / 13 / 1996$ & TWDB, 2009 \\
\hline AY-68-28-706 & 742 & 875 & 722 & 153 & report/publication & USGS & -- & Small, 1984 \\
\hline AY-68-28-708 & 403 & 900 & 735 & 165 & drillers' log & TWDB & $10 / 8 / 1966$ & TWDB, 2009 \\
\hline AY-68-28-7hh & 405 & 789 & 617 & 172 & report/publication & TWDB & -- & Livingston, 1947 \\
\hline AY-68-28-7ii & 368 & 884 & 728 & 156 & geophysical log & TWDB & $1 / 16 / 1990$ & unpublished data, 1990 \\
\hline AY-68-28-7kk & 355 & 823 & 669 & 154 & geophysical log & TWDB & $10 / 6 / 1999$ & unpublished data, 1999 \\
\hline AY-68-28-711 & 431 & 730 & 598 & 132 & geophysical log & TWDB & $5 / 1 / 1989$ & unpublished data, 1989 \\
\hline AY-68-28-7nn & 371 & 758 & 632 & 126 & geophysical log & TWDB & $7 / 27 / 1998$ & unpublished data, 1998 \\
\hline AY-68-28-7oo & 370 & 890 & 736 & 154 & geophysical log & TWDB & $5 / 11 / 1994$ & unpublished data, 1994 \\
\hline AY-68-28-7pp & 511 & 711 & 553 & 158 & geophysical log & TWDB & $3 / 13 / 1995$ & unpublished data, 1995 \\
\hline $\mathrm{AY}-68-28-7 \mathrm{qq}$ & 337 & 915 & 755 & 160 & geophysical log & TWDB & 3/9/1994 & unpublished data, 1994 \\
\hline
\end{tabular}


Appendix 1. Wells selected for inclusion in geodatabase of selected wells penetrating the Austin Group, central Bexar County, Texas,

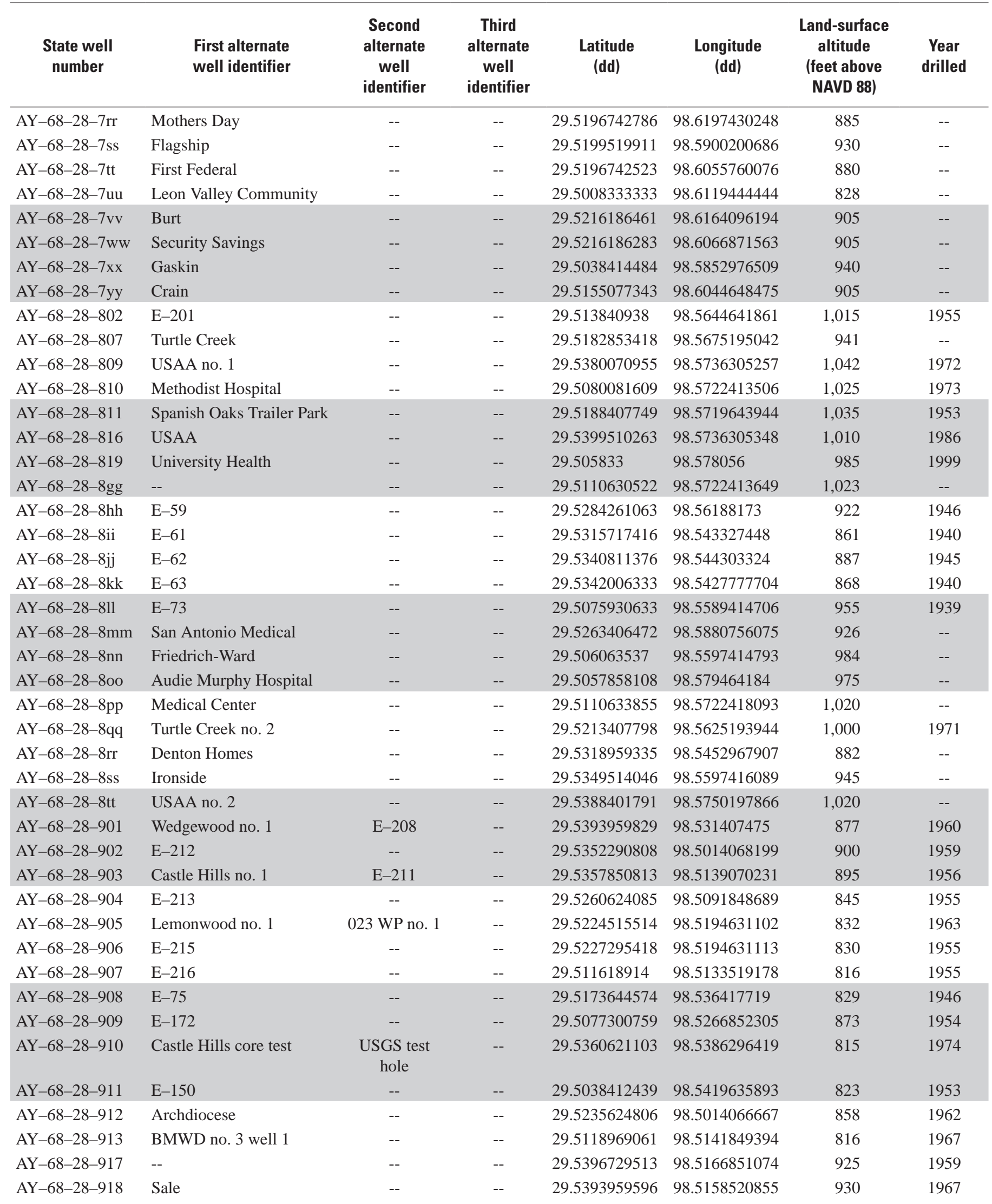




\begin{tabular}{|c|c|c|c|c|c|c|c|c|}
\hline $\begin{array}{l}\text { State well } \\
\text { number }\end{array}$ & $\begin{array}{l}\text { Well depth } \\
\text { (feet } \\
\text { below } \\
\text { LSD) }\end{array}$ & $\begin{array}{l}\text { Altitude of } \\
\text { top of Aus- } \\
\text { tin Group } \\
\text { (feet above } \\
\text { NAVD 88) }\end{array}$ & $\begin{array}{c}\text { Altitude of } \\
\text { base of Aus- } \\
\text { tin Group } \\
\text { (feet above } \\
\text { NAVD 88) }\end{array}$ & $\begin{array}{l}\text { Thickness } \\
\text { of Austin } \\
\text { Group } \\
\text { (feet) }\end{array}$ & Data source & Source agency & $\begin{array}{c}\text { Data } \\
\text { source date }\end{array}$ & $\begin{array}{c}\text { Report } \\
\text { author, } \\
\text { year }^{1}\end{array}$ \\
\hline AY-68-28-7rr & 369 & 885 & 731 & 154 & geophysical log & TWDB & 9/9/1998 & unpublished data, 1998 \\
\hline AY-68-28-7ss & 442 & 800 & 636 & 164 & geophysical log & TWDB & $8 / 15 / 1994$ & unpublished data, 1994 \\
\hline AY-68-28-7tt & 390 & 870 & 710 & 160 & geophysical log & TWDB & $10 / 25 / 1989$ & unpublished data, 1989 \\
\hline AY-68-28-7uu & 258 & 798 & 646 & 152 & geophysical log & TWDB & $6 / 2 / 2002$ & unpublished data, 2002 \\
\hline AY-68-28-7vv & 246 & 905 & 747 & 158 & geophysical log & TWDB & 6/7/1991 & unpublished data, 1991 \\
\hline AY-68-28-7ww & 425 & 881 & 721 & 160 & geophysical log & TWDB & $1 / 8 / 1990$ & unpublished data, 1990 \\
\hline AY-68-28-7xx & 447 & 806 & 660 & 146 & geophysical log & TWDB & $8 / 21 / 1989$ & unpublished data, 1989 \\
\hline AY-68-28-7yy & 279 & 855 & 697 & 158 & geophysical log & TWDB & $5 / 29 / 1990$ & unpublished data, 1990 \\
\hline AY-68-28-802 & 590 & 803 & 648 & 155 & drillers' log & TWDB & 9/1/1955 & TWDB, 2009 \\
\hline AY-68-28-807 & 548 & 815 & 666 & 149 & drillers' log & TWDB & $3 / 26 / 1971$ & TWDB, 2009 \\
\hline AY-68-28-809 & 750 & 831 & 674 & 157 & geophysical log & USGS & $12 / 5 / 1972$ & unpublished data, 1972 \\
\hline AY-68-28-810 & 654 & 827 & 653 & 174 & geophysical log & USGS & $10 / 23 / 1973$ & unpublished data, 1973 \\
\hline AY-68-28-811 & 612 & 819 & 663 & 156 & drillers' log & SAWS & $5 / 6 / 1905$ & unpublished data, 1905 \\
\hline AY-68-28-816 & 742 & 805 & 645 & 160 & drillers' log & TWDB & $5 / 1 / 1986$ & TWDB, 2009 \\
\hline AY-68-28-819 & 780 & 738 & 655 & 83 & drillers' log & TWDB & $12 / 29 / 1999$ & TWDB, 2009 \\
\hline AY-68-28-8gg & -- & 803 & 649 & 154 & gamma & USGS & -- & Small, 1984 \\
\hline AY-68-28-8hh & 325 & 919 & 769 & 150 & report/publication & TWDB & -- & Livingston, 1947 \\
\hline AY-68-28-8ii & 323 & 861 & 710 & 151 & report/publication & TWDB & -- & Livingston, 1947 \\
\hline AY-68-28-8jj & 300 & 887 & 762 & 125 & report/publication & TWDB & -- & Livingston, 1947 \\
\hline AY-68-28-8kk & 270 & 868 & 752 & 116 & report/publication & TWDB & -- & Livingston, 1947 \\
\hline AY-68-28-811 & 467 & 799 & 653 & 146 & report/publication & TWDB & -- & Livingston, 1947 \\
\hline AY-68-28-8mm & -- & 870 & 716 & 154 & geophysical log & TWDB & $2 / 5 / 2001$ & unpublished data, 2001 \\
\hline AY-68-28-8nn & 514 & 846 & 692 & 154 & geophysical log & TWDB & $7 / 2 / 1986$ & unpublished data, 1986 \\
\hline AY-68-28-80o & 466 & 813 & 653 & 160 & geophysical log & TWDB & 3/1/1984 & unpublished data, 1984 \\
\hline AY-68-28-8pp & 508 & 800 & 646 & 154 & geophysical log & USGS & $3 / 2 / 1978$ & Small, 1984 \\
\hline AY-68-28-8qq & 685 & 1,000 & 830 & 170 & geophysical log & TWDB & 9/16/1982 & unpublished data, 1982 \\
\hline AY-68-28-8rr & 258 & 882 & 742 & 140 & geophysical log & TWDB & 4/2/1986 & unpublished data, 1986 \\
\hline AY-68-28-8ss & 383 & 870 & 731 & 139 & geophysical log & TWDB & $9 / 23 / 1982$ & unpublished data, 1982 \\
\hline AY-68-28-8tt & 736 & 820 & 665 & 155 & geophysical log & TWDB & $5 / 5 / 1986$ & unpublished data, 1986 \\
\hline AY-68-28-901 & 791 & 877 & 723 & 154 & geophysical log & SAWS & $3 / 25 / 1960$ & unpublished data, 1960 \\
\hline AY-68-28-902 & 881 & 819 & 663 & 156 & drillers' log & TWDB & $7 / 1 / 1959$ & TWDB, 2009 \\
\hline AY-68-28-903 & 762 & 835 & 675 & 160 & drillers' log & EAA & $5 / 19 / 1956$ & unpublished data, 1956 \\
\hline AY-68-28-904 & 640 & 630 & 455 & 175 & drillers' log & BMWD & $5 / 1 / 1955$ & unpublished data, 1955 \\
\hline AY-68-28-905 & 856 & 545 & 431 & 114 & drillers' log & BMWD & $1 / 3 / 1983$ & unpublished data, 1983 \\
\hline AY-68-28-906 & 528 & 685 & 550 & 135 & drillers' log & BMWD & $1 / 1 / 1955$ & unpublished data, 1955 \\
\hline AY-68-28-907 & 533 & 657 & 530 & 127 & drillers' log & BMWD & $1 / 1 / 1955$ & unpublished data, 1955 \\
\hline AY-68-28-908 & 325 & 829 & 620 & 209 & report/publication & TWDB & -- & Livingston, 1947 \\
\hline AY-68-28-909 & 867 & 773 & 668 & 105 & drillers' log & SAWS & $7 / 21 / 1954$ & Petitt and George, 1956 \\
\hline AY-68-28-910 & 804 & 815 & 745 & 70 & geophysical log & USGS & $4 / 24 / 1974$ & unpublished data, 1974 \\
\hline AY-68-28-911 & 850 & 743 & 587 & 156 & geophysical log & SAWS & $5 / 12 / 1953$ & Petitt and George, 1956 \\
\hline AY-68-28-912 & 537 & 671 & 525 & 146 & drillers' log & TWDB & 9/1/1962 & TWDB, 2009 \\
\hline AY-68-28-913 & 787 & 655 & 514 & 141 & drillers' log & BMWD & $5 / 30 / 1957$ & unpublished data, 1957 \\
\hline AY-68-28-917 & 392 & 870 & 740 & 130 & drillers' log & TWDB & -- & TWDB, 2009 \\
\hline AY-68-28-918 & 400 & 930 & 745 & 185 & drillers' log & TWDB & $11 / 1 / 1967$ & TWDB, 2009 \\
\hline
\end{tabular}


Appendix 1. Wells selected for inclusion in geodatabase of selected wells penetrating the Austin Group, central Bexar County, Texas,

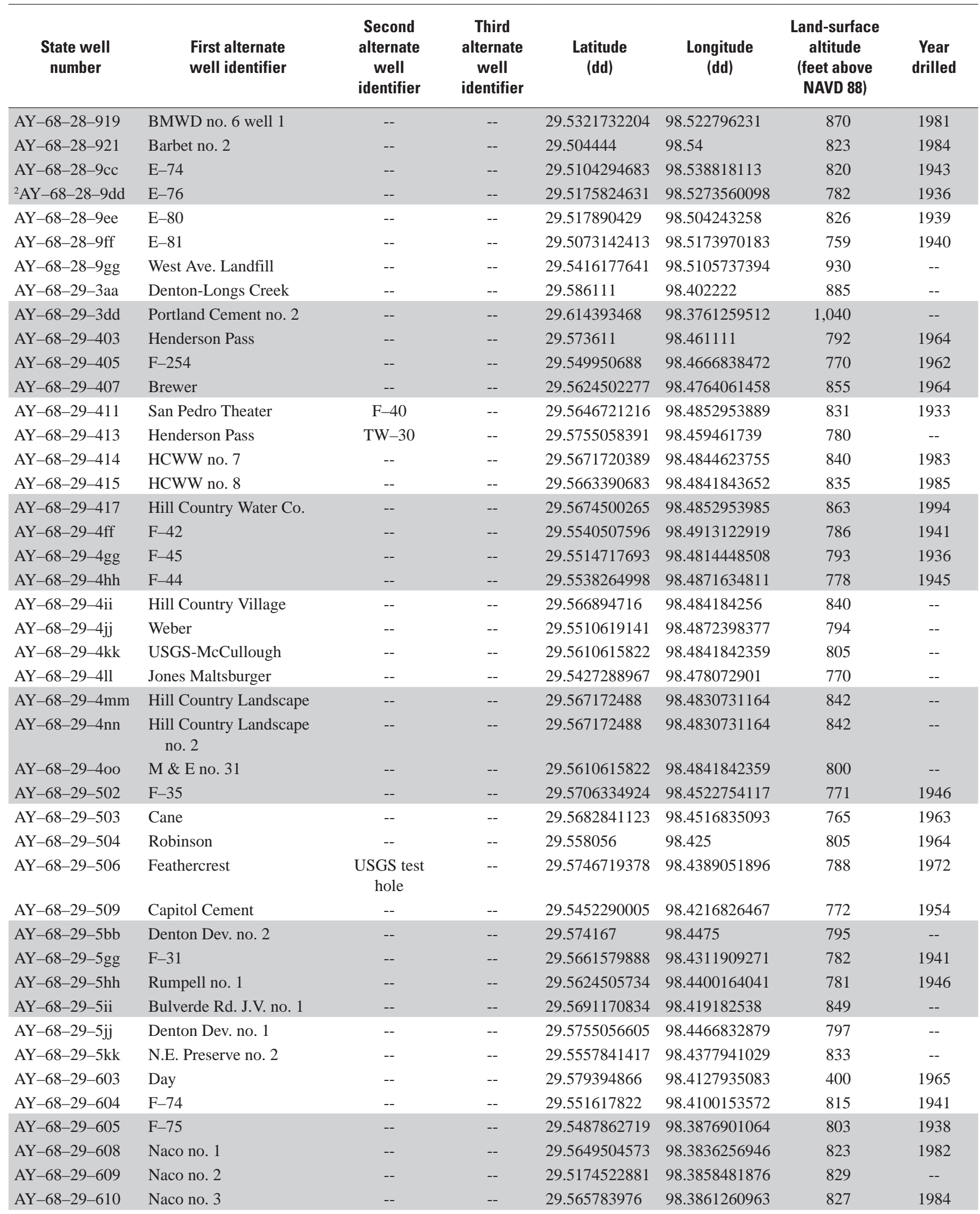




\begin{tabular}{|c|c|c|c|c|c|c|c|c|}
\hline $\begin{array}{l}\text { State well } \\
\text { number }\end{array}$ & $\begin{array}{c}\text { Well depth } \\
\text { (feet } \\
\text { below } \\
\text { LSD) }\end{array}$ & $\begin{array}{l}\text { Altitude of } \\
\text { top of Aus- } \\
\text { tin Group } \\
\text { (feet above } \\
\text { NAVD 88) }\end{array}$ & $\begin{array}{l}\text { Altitude of } \\
\text { base of Aus- } \\
\text { tin Group } \\
\text { (feet above } \\
\text { NAVD 88) }\end{array}$ & $\begin{array}{c}\text { Thickness } \\
\text { of Austin } \\
\text { Group } \\
\text { (feet) }\end{array}$ & Data source & Source agency & $\begin{array}{c}\text { Data } \\
\text { source date }\end{array}$ & $\begin{array}{c}\text { Report } \\
\text { author, } \\
\text { year }^{1}\end{array}$ \\
\hline AY-68-28-919 & 550 & 870 & 755 & 115 & drillers' log & BMWD & $4 / 23 / 1981$ & unpublished data, 1981 \\
\hline AY-68-28-921 & 855 & 741 & 603 & 138 & geophysical log & SAWS & 2/1/1984 & unpublished data, 1984 \\
\hline AY-68-28-9cc & 436 & 740 & 571 & 169 & report/publication & TWDB & -- & Livingston, 1947 \\
\hline${ }^{2} \mathrm{AY}-68-28-9 \mathrm{dd}$ & 282 & -- & -- & -- & report/publication & TWDB & -- & Livingston, 1947 \\
\hline AY-68-28-9ee & 498 & 580 & 423 & 157 & report/publication & TWDB & -- & Livingston, 1947 \\
\hline AY-68-28-9ff & 390 & 682 & 536 & 146 & report/publication & TWDB & -- & Livingston, 1947 \\
\hline AY-68-28-9gg & 120 & 930 & 800 & 130 & geophysical log & TWDB & $10 / 6 / 1983$ & unpublished data, 1983 \\
\hline AY-68-29-3aа & -- & 671 & 539 & 132 & geophysical log & EAA & $6 / 26 / 1989$ & unpublished data, 1989 \\
\hline AY-68-29-3dd & 650 & 1,040 & 918 & 122 & geophysical log & TWDB & $6 / 26 / 1980$ & unpublished data, 1980 \\
\hline AY-68-29-403 & 340 & 792 & 682 & 110 & drillers' log & USGS & $8 / 23 / 1968$ & Small, 1984 \\
\hline AY-68-29-405 & 395 & 770 & 600 & 170 & drillers' log & TWDB & $6 / 1 / 1962$ & TWDB, 2009 \\
\hline AY-68-29-407 & 349 & 855 & 750 & 105 & drillers' log & TWDB & $6 / 1 / 1964$ & TWDB, 2009 \\
\hline AY-68-29-411 & 415 & 731 & 611 & 120 & drillers' log & TWDB & $4 / 16 / 1905$ & Petitt and George, 1956 \\
\hline AY-68-29-413 & -- & 780 & 712 & 68 & geophysical log & EAA & $11 / 28 / 1988$ & unpublished data, 1988 \\
\hline AY-68-29-414 & 710 & 723 & 583 & 140 & drillers' log & TWDB & $2 / 25 / 1983$ & TWDB, 2009 \\
\hline AY-68-29-415 & 1,078 & 673 & 537 & 136 & geophysical log & EAA & 7/18/1986 & unpublished data, 1986 \\
\hline AY-68-29-417 & 415 & 752 & 603 & 149 & drillers' log & TWDB & 3/1/1994 & TWDB, 2009 \\
\hline AY-68-29-4ff & 240 & 786 & 671 & 115 & report/publication & TWDB & -- & Livingston, 1947 \\
\hline AY-68-29-4gg & 190 & 793 & 673 & 120 & report/publication & TWDB & -- & Livingston, 1947 \\
\hline AY-68-29-4hh & 245 & 778 & 673 & 105 & report/publication & TWDB & -- & Livingston, 1947 \\
\hline AY-68-29-4ii & 661 & 724 & 586 & 138 & geophysical log & TWDB & $3 / 2 / 1983$ & unpublished data, 1983 \\
\hline AY-68-29-4jj & 228 & 794 & 770 & 24 & geophysical log & TWDB & $10 / 23 / 1992$ & unpublished data, 1992 \\
\hline AY-68-29-4kk & 315 & 805 & 677 & 128 & geophysical log & TWDB & $8 / 23 / 1978$ & unpublished data, 1978 \\
\hline AY-68-29-411 & 260 & 770 & 634 & 136 & geophysical log & TWDB & $1 / 21 / 1981$ & unpublished data, 1981 \\
\hline AY-68-29-4mm & 299 & 722 & 595 & 127 & geophysical log & TWDB & $1 / 20 / 1986$ & unpublished data, 1986 \\
\hline AY-68-29-4nn & 299 & 730 & 595 & 135 & geophysical log & TWDB & $1 / 20 / 1986$ & unpublished data, 1986 \\
\hline AY-68-29-4oo & 737 & 800 & 672 & 128 & geophysical log & TWDB & $8 / 23 / 1981$ & unpublished data, 1981 \\
\hline AY-68-29-502 & 264 & 771 & 681 & 90 & report/publication & TWDB & -- & Livingston, 1947 \\
\hline AY-68-29-503 & 349 & 753 & 658 & 95 & drillers' log & TWDB & $5 / 1 / 1963$ & TWDB, 2009 \\
\hline AY-68-29-504 & 305 & 770 & 634 & 136 & drillers' log & TWDB & 3/1/1964 & TWDB, 2009 \\
\hline AY-68-29-506 & 694 & 788 & 696 & 92 & geophysical log & USGS & $10 / 26 / 1972$ & unpublished data, 1972 \\
\hline AY-68-29-509 & 725 & 637 & 498 & 139 & drillers' log & TWDB & $10 / 2 / 1954$ & TWDB, 2009 \\
\hline AY-68-29-5bb & -- & 795 & 752 & 43 & geophysical log & EAA & $8 / 18 / 1992$ & unpublished data, 1992 \\
\hline AY-68-29-5gg & 300 & 782 & 677 & 105 & report/publication & TWDB & -- & Livingston, 1947 \\
\hline AY-68-29-5hh & 264 & 781 & 655 & 126 & geophysical log & TWDB & $5 / 31 / 1979$ & unpublished data, 1979 \\
\hline AY-68-29-5ii & 401 & 789 & 653 & 136 & drillers' log & EAA & $6 / 26 / 1989$ & unpublished data, 1989 \\
\hline AY-68-29-5jj & 265 & 797 & 771 & 26 & geophysical log & TWDB & 9/18/1992 & unpublished data, 1992 \\
\hline AY-68-29-5kk & 337 & 763 & 629 & 134 & geophysical log & TWDB & $6 / 22 / 1979$ & unpublished data, 1979 \\
\hline AY-68-29-603 & 480 & 232 & 158 & 74 & drillers' log & TWDB & $4 / 14 / 1965$ & TWDB, 2009 \\
\hline AY-68-29-604 & 457 & 613 & 499 & 114 & drillers' log & TWDB & $12 / 3 / 1941$ & TWDB, 2009 \\
\hline AY-68-29-605 & 438 & 755 & 603 & 152 & report/publication & TWDB & -- & Livingston, 1947 \\
\hline AY-68-29-608 & 819 & 673 & 535 & 138 & geophysical log & SAWS & $8 / 2 / 1982$ & unpublished data, 1982 \\
\hline AY-68-29-609 & 833 & 749 & 604 & 145 & geophysical log & TWDB & $11 / 5 / 1981$ & TWDB, 2009 \\
\hline AY-68-29-610 & 811 & 744 & 607 & 137 & drillers' log & TWDB & 6/6/1905 & TWDB, 2009 \\
\hline
\end{tabular}


Appendix 1. Wells selected for inclusion in geodatabase of selected wells penetrating the Austin Group, central Bexar County, Texas,

\begin{tabular}{|c|c|c|c|c|c|c|c|}
\hline $\begin{array}{c}\text { State well } \\
\text { number }\end{array}$ & $\begin{array}{l}\text { First alternate } \\
\text { well identifier }\end{array}$ & $\begin{array}{c}\text { Second } \\
\text { alternate } \\
\text { well } \\
\text { identifier }\end{array}$ & $\begin{array}{c}\text { Third } \\
\text { alternate } \\
\text { well } \\
\text { identifier }\end{array}$ & $\begin{array}{l}\text { Latitude } \\
\text { (dd) }\end{array}$ & $\begin{array}{l}\text { Longitude } \\
\text { (dd) }\end{array}$ & $\begin{array}{l}\text { Land-surface } \\
\text { altitude } \\
\text { (feet above } \\
\text { NAVD 88) }\end{array}$ & $\begin{array}{l}\text { Year } \\
\text { drilled }\end{array}$ \\
\hline AY-68-29-611 & Naco no. 4 & -- & -- & 29.5663394053 & 98.385014735 & 830 & 1985 \\
\hline AY-68-29-612 & Naco no. 5 & -- & -- & 29.5647222222 & 98.3855555556 & 818 & -- \\
\hline AY-68-29-6аa & $\mathrm{F}-72$ & -- & -- & 29.5460979636 & 98.4135977616 & 772 & 1946 \\
\hline AY-68-29-6bb & $\mathrm{F}-27$ & -- & -- & 29.572215821 & 98.3963381739 & 891 & 1945 \\
\hline AY-68-29-701 & Fairchild & $\mathrm{F}-172$ & -- & 29.5377291132 & 98.4644617488 & 778 & 1942 \\
\hline AY-68-29-702 & CY-319 & -- & -- & 29.5232855397 & 98.4847392316 & 787 & 1965 \\
\hline AY-68-29-703 & Q-69 & -- & -- & 29.5227295615 & 98.483906319 & 770 & 1967 \\
\hline AY-68-29-707 & Maltsberger no. 1 & -- & -- & 29.5232851008 & 98.4830729658 & 766 & -- \\
\hline AY-68-29-709 & $\mathrm{F}-63$ & -- & -- & 29.5032558658 & 98.463728309 & 829 & -- \\
\hline AY-68-29-711 & $\mathrm{F}-128$ & -- & -- & 29.5255074931 & 98.4758510068 & 795 & 1945 \\
\hline AY-68-29-712 & Maltsberger no. 4 & -- & -- & 29.5232855434 & 98.4836282026 & 775 & 1985 \\
\hline AY-68-29-714 & Maltsberger no. 5 & -- & -- & 29.5227295642 & 98.4830731862 & 758 & 1997 \\
\hline AY-68-29-7cc & $\mathrm{F}-49$ & -- & -- & 29.5377919086 & 98.4638404398 & 748 & 1942 \\
\hline AY-68-29-7dd & F-51 & -- & -- & 29.5253623455 & 98.4976028172 & 856 & 1941 \\
\hline AY-68-29-7ee & $\mathrm{F}-52$ & -- & -- & 29.5337552283 & 98.4844723607 & 823 & 1941 \\
\hline AY-68-29-7ff & F-57 & -- & -- & 29.5250287668 & 98.472928593 & 787 & 1941 \\
\hline AY-68-29-7gg & F-64 & -- & -- & 29.5062833761 & 98.4613735794 & 829 & 1945 \\
\hline AY-68-29-7hh & Maltsberger no. 6 & -- & -- & 29.522451795 & 98.483350748 & 758 & -- \\
\hline AY-68-29-803 & $\mathrm{F}-229$ & -- & -- & 29.5316183599 & 98.419738001 & 777 & 1961 \\
\hline AY-68-29-804 & Perrin no. 3 & $\mathrm{~F}-230$ & -- & 29.5313404759 & 98.4211265922 & 763 & 1954 \\
\hline AY-68-29-805 & $\mathrm{F}-231$ & -- & -- & 29.5310624863 & 98.4208495841 & 768 & 1961 \\
\hline AY-68-29-806 & Rooke & -- & -- & 29.5041193248 & 98.4452941414 & 780 & 1962 \\
\hline AY-68-29-811 & $\mathrm{F}-190$ & -- & -- & 29.5138410939 & 98.4483498066 & 736 & 1954 \\
\hline AY-68-29-812 & Turner & -- & -- & 29.5243966398 & 98.443072146 & 780 & 1972 \\
\hline AY-68-29-813 & $\mathrm{F}-142$ & -- & -- & 29.5327294378 & 98.4183495233 & 780 & 1950 \\
\hline AY-68-29-816 & $\mathrm{F}-89$ & -- & -- & 29.5053863356 & 98.4323319068 & 700 & 1944 \\
\hline AY-68-29-8aa & F-66 & -- & -- & 29.5216451875 & 98.4438812974 & 776 & 1941 \\
\hline AY-68-29-8bb & F-69 & -- & -- & 29.5176085073 & 98.4247070667 & 729 & 1942 \\
\hline AY-68-29-8cc & F-70 & -- & -- & 29.5181691571 & 98.4222402059 & 733 & 1941 \\
\hline AY-68-29-8dd & $\mathrm{F}-65$ & -- & -- & 29.5172721169 & 98.4435449081 & 748 & 1946 \\
\hline AY-68-29-912 & W.C.I.D. no. 10 & -- & -- & 29.5110632541 & 98.3947368471 & 748 & 1967 \\
\hline AY-68-29-913 & Randolph no. 1 & -- & -- & 29.5310629574 & 98.3794591661 & 822 & 1968 \\
\hline AY-68-29-914 & N. Alamo Height no. 1 & Walzem & -- & 29.508008327 & 98.4041820846 & 737 & 1955 \\
\hline AY-68-29-915 & Randolph no. 2 & -- & -- & 29.5313406165 & 98.3789034856 & 815 & 1971 \\
\hline AY-68-29-918 & $\mathrm{F}-248$ & -- & -- & 29.518007845 & 98.3797369185 & 790 & 1960 \\
\hline AY-68-29-920 & Windcrest no. 3 & -- & -- & 29.5168967918 & 98.3736256452 & 790 & 1963 \\
\hline AY-68-29-925 & Interchange Plaza & -- & -- & 29.515556 & 98.398056 & 753 & 1987 \\
\hline AY-68-29-926 & Randolph no. 4 & -- & -- & 29.531111 & 98.378889 & 812 & 1992 \\
\hline AY-68-29-929 & Randolph no. 3 & -- & -- & 29.529444 & 98.378611 & 813 & 1992 \\
\hline AY-68-29-9ff & F-71 & -- & -- & 29.5412735618 & 98.4139257174 & 792 & 1946 \\
\hline AY-68-29-9gg & $\mathrm{F}-85$ & -- & -- & 29.5175132454 & 98.3990236716 & 744 & 1940 \\
\hline AY-68-29-9hh & $\mathrm{F}-88$ & -- & -- & 29.5086381061 & 98.412372765 & 708 & 1940 \\
\hline AY-68-29-9ii & Artesia & -- & -- & 29.514119027 & 98.3983485048 & 742 & -- \\
\hline AY-68-29-9jj & Gill Savings & -- & -- & 29.5163411745 & 98.3980707257 & 752 & -- \\
\hline
\end{tabular}


2010-Continued.

\begin{tabular}{|c|c|c|c|c|c|c|c|c|}
\hline $\begin{array}{c}\text { State well } \\
\text { number }\end{array}$ & $\begin{array}{l}\text { Well depth } \\
\text { (feet } \\
\text { below } \\
\text { LSD) }\end{array}$ & $\begin{array}{c}\text { Altitude of } \\
\text { top of Aus- } \\
\text { tin Group } \\
\text { (feet above } \\
\text { NAVD 88) }\end{array}$ & $\begin{array}{l}\text { Altitude of } \\
\text { base of Aus- } \\
\text { tin Group } \\
\text { (feet above } \\
\text { NAVD 88) }\end{array}$ & $\begin{array}{c}\text { Thickness } \\
\text { of Austin } \\
\text { Group } \\
\text { (feet) }\end{array}$ & Data source & Source agency & $\begin{array}{c}\text { Data } \\
\text { source date }\end{array}$ & $\begin{array}{c}\text { Report } \\
\text { author, } \\
\text { year }^{1}\end{array}$ \\
\hline AY-68-29-611 & 785 & 746 & 610 & 136 & geophysical log & SAWS & $5 / 28 / 1985$ & unpublished data, 1985 \\
\hline AY-68-29-612 & 840 & 748 & 608 & 140 & geophysical log & TWDB & $8 / 8 / 1965$ & TWDB, 2009 \\
\hline AY-68-29-6аa & 455 & 624 & 468 & 156 & report/publication & TWDB & -- & Livingston, 1947 \\
\hline AY-68-29-6bb & 682 & 756 & 617 & 139 & report/publication & TWDB & -- & Livingston, 1947 \\
\hline AY-68-29-701 & 500 & 616 & 480 & 136 & geophysical log & EAA & $5 / 15 / 1992$ & Petitt and George, 1956 \\
\hline AY-68-29-702 & 866 & 622 & 479 & 143 & drillers' log & SAWS & $3 / 1 / 1965$ & unpublished data, 1965 \\
\hline AY-68-29-703 & 824 & 770 & 458 & 312 & drillers' log & TWDB & $11 / 3 / 1967$ & TWDB, 2009 \\
\hline AY-68-29-707 & 857 & 606 & 458 & 148 & geophysical log & TWDB & $1 / 24 / 1965$ & TWDB, 2009 \\
\hline AY-68-29-709 & 339 & 799 & 642 & 157 & report/publication & TWDB & -- & Livingston, 1947 \\
\hline AY-68-29-711 & 600 & 600 & 453 & 147 & geophysical log & TWDB & $8 / 20 / 1947$ & Petitt and George, 1956 \\
\hline AY-68-29-712 & 851 & 637 & 485 & 152 & drillers' log & SAWS & $1 / 19 / 1985$ & unpublished data, 1985 \\
\hline AY-68-29-714 & 848 & 612 & 480 & 132 & geophysical log & EAA & $5 / 23 / 1986$ & unpublished data, 1986 \\
\hline AY-68-29-7cc & 402 & 693 & 555 & 138 & report/publication & TWDB & -- & Livingston, 1947 \\
\hline AY-68-29-7dd & 502 & 632 & 494 & 138 & report/publication & TWDB & -- & Livingston, 1947 \\
\hline AY-68-29-7ee & 266 & 691 & 564 & 127 & report/publication & TWDB & -- & Livingston, 1947 \\
\hline AY-68-29-7ff & 461 & 695 & 538 & 157 & report/publication & TWDB & -- & Livingston, 1947 \\
\hline AY-68-29-7gg & 348 & 829 & 664 & 165 & report/publication & TWDB & -- & Livingston, 1947 \\
\hline AY-68-29-7hh & 844 & 603 & 478 & 125 & geophysical log & TWDB & 4/18/1997 & unpublished data, 1997 \\
\hline AY-68-29-803 & 795 & 777 & 668 & 109 & drillers' log & TWDB & $10 / 2 / 1961$ & TWDB, 2009 \\
\hline AY-68-29-804 & 761 & 763 & 656 & 107 & geophysical log & TWDB & $11 / 8 / 1954$ & TWDB, 2009 \\
\hline AY-68-29-805 & 800 & 768 & 659 & 109 & drillers' log & TWDB & 1/1/1961 & TWDB, 2009 \\
\hline AY-68-29-806 & 398 & 780 & 639 & 141 & drillers' log & TWDB & 8/1/1962 & TWDB, 2009 \\
\hline AY-68-29-811 & 612 & 736 & 636 & 100 & drillers' log & TWDB & 1/1/1954 & Petitt and George, 1956 \\
\hline AY-68-29-812 & 350 & 780 & 717 & 63 & drillers' log & SAWS & $6 / 15 / 1972$ & unpublished data, 1972 \\
\hline AY-68-29-813 & 369 & 780 & 657 & 123 & geophysical log & TWDB & $4 / 20 / 1950$ & Petitt and George, 1956 \\
\hline AY-68-29-816 & 347 & 700 & 532 & 168 & report/publication & TWDB & -- & Livingston, 1947 \\
\hline AY-68-29-8аa & 318 & 776 & 659 & 117 & report/publication & TWDB & -- & Livingston, 1947 \\
\hline AY-68-29-8bb & 284 & 729 & 606 & 123 & report/publication & TWDB & -- & Livingston, 1947 \\
\hline AY-68-29-8cc & 286 & 733 & 608 & 125 & report/publication & TWDB & -- & Livingston, 1947 \\
\hline AY-68-29-8dd & 359 & 748 & 633 & 115 & report/publication & TWDB & -- & Livingston, 1947 \\
\hline AY-68-29-912 & 630 & 563 & 458 & 105 & drillers' log & TWDB & $11 / 6 / 1967$ & TWDB, 2009 \\
\hline AY-68-29-913 & 784 & 822 & 586 & 236 & geophysical log & TWDB & $7 / 25 / 1968$ & TWDB, 2009 \\
\hline AY-68-29-914 & 845 & 597 & 467 & 130 & geophysical log & TWDB & $2 / 22 / 1955$ & TWDB, 2009 \\
\hline AY-68-29-915 & 824 & 676 & 560 & 116 & geophysical log & SAWS & $11 / 30 / 1971$ & unpublished data, 1971 \\
\hline AY-68-29-918 & 690 & 680 & 530 & 150 & drillers' log & TWDB & $5 / 1 / 1960$ & TWDB, 2009 \\
\hline AY-68-29-920 & 655 & 590 & 445 & 145 & drillers' log & TWDB & $1 / 1 / 1963$ & TWDB, 2009 \\
\hline AY-68-29-925 & 525 & 714 & 536 & 178 & drillers' log & EAA & 4/10/1987 & unpublished data, 1987 \\
\hline AY-68-29-926 & 870 & 688 & 552 & 136 & drillers' log & TWDB & 4/4/1992 & TWDB, 2009 \\
\hline AY-68-29-929 & 870 & 689 & 553 & 136 & drillers' log & TWDB & $2 / 22 / 1992$ & TWDB, 2009 \\
\hline AY-68-29-9ff & 315 & 792 & 624 & 168 & report/publication & TWDB & -- & Livingston, 1947 \\
\hline AY-68-29-9gg & 442 & 642 & 488 & 154 & report/publication & TWDB & -- & Livingston, 1947 \\
\hline AY-68-29-9hh & 376 & 665 & 498 & 167 & report/publication & TWDB & -- & Livingston, 1947 \\
\hline AY-68-29-9ii & 512 & 618 & 496 & 122 & geophysical log & TWDB & 6/9/1986 & unpublished data, 1986 \\
\hline AY-68-29-9jj & 438 & 690 & 534 & 156 & geophysical log & TWDB & $5 / 23 / 1986$ & unpublished data, 1986 \\
\hline
\end{tabular}


Appendix 1. Wells selected for inclusion in geodatabase of selected wells penetrating the Austin Group, central Bexar County, Texas,

\begin{tabular}{|c|c|c|c|c|c|c|c|}
\hline $\begin{array}{l}\text { State well } \\
\text { number }\end{array}$ & $\begin{array}{l}\text { First alternate } \\
\text { well identifier }\end{array}$ & $\begin{array}{l}\text { Second } \\
\text { alternate } \\
\text { well } \\
\text { identifier }\end{array}$ & $\begin{array}{c}\text { Third } \\
\text { alternate } \\
\text { well } \\
\text { identifier }\end{array}$ & $\begin{array}{l}\text { Latitude } \\
\text { (dd) }\end{array}$ & $\begin{array}{l}\text { Longitude } \\
\text { (dd) }\end{array}$ & $\begin{array}{l}\text { Land-surface } \\
\text { altitude } \\
\text { (feet above } \\
\text { NAVD 88) }\end{array}$ & $\begin{array}{l}\text { Year } \\
\text { drilled }\end{array}$ \\
\hline AY-68-29-9kk & Gill Savings no. 2 & -- & -- & 29.5174522423 & 98.3997374388 & 748 & -- \\
\hline AY-68-29-911 & Swans no. 2 & -- & -- & 29.5241186257 & 98.4166823441 & 753 & -- \\
\hline AY-68-29-9mm & Standard & -- & -- & 29.5193966288 & 98.3972373787 & 750 & -- \\
\hline AY-68-30-105 & $\mathrm{F}-250$ & -- & -- & 29.5927276465 & 98.3447357244 & 820 & 1962 \\
\hline AY-68-30-106 & Hastey & -- & -- & 29.6068941859 & 98.3389025985 & 800 & 1968 \\
\hline AY-68-30-107 & Pope & -- & -- & 29.5977274921 & 98.3402916162 & 788 & 1970 \\
\hline AY-68-30-109 & Foxrun & -- & -- & 29.5896716937 & 98.3611251558 & 947 & 1973 \\
\hline AY-68-30-1aa & $\mathrm{F}-25$ & -- & -- & 29.5935120882 & 98.3586231318 & 903 & 1941 \\
\hline AY-68-30-1bb & Khakish & -- & -- & 29.5841167637 & 98.3633477577 & 920 & -- \\
\hline AY-68-30-212 & Fey & -- & -- & 29.594116701 & 98.3152909391 & 771 & 1968 \\
\hline AY-68-30-213 & Boysville & -- & -- & 29.591616733 & 98.330569343 & 780 & 1963 \\
\hline AY-68-30-214 & $\mathrm{G}-71$ & -- & -- & 29.5882839091 & 98.3122348462 & 761 & 1962 \\
\hline AY-68-30-220 & Gose & -- & -- & 29.6013384543 & 98.3158469679 & 770 & 1975 \\
\hline AY-68-30-401 & W.C.I.D. no. 13 & $\mathrm{~F}-256$ & -- & 29.5585618363 & 98.3358474145 & 899 & 1965 \\
\hline AY-68-30-402 & Live Oak Village & -- & -- & 29.5566178731 & 98.344458638 & 885 & 1969 \\
\hline AY $-68-30-403$ & Village Public Utility & -- & -- & 29.544951285 & 98.3397354882 & 874 & 1972 \\
\hline AY-68-30-404 & $\mathrm{F}-81$ & -- & -- & 29.5460621755 & 98.361125054 & 946 & 1939 \\
\hline AY-68-30-405 & Valley Forge & -- & -- & 29.5499510059 & 98.3722363561 & 848 & 1973 \\
\hline AY-68-30-406 & Green Meadows & -- & -- & 29.5652285903 & 98.3416805832 & 869 & 1967 \\
\hline$A Y-68-30-4 b b$ & Jordan Ford no. 2 & -- & -- & 29.5591176838 & 98.3425138143 & 880 & -- \\
\hline AY-68-30-506 & Universal City & -- & -- & 29.5510625186 & 98.3077906584 & 838 & 1967 \\
\hline AY-68-30-510 & G-66 & -- & -- & 29.5535621221 & 98.3014014943 & 835 & 1952 \\
\hline AY-68-30-511 & $\mathrm{G}-70$ & -- & -- & 29.5513403122 & 98.300290461 & 825 & 1963 \\
\hline AY-68-30-512 & Live Oak well no. 6 & -- & -- & 29.5693955113 & 98.3233471055 & 840 & 1971 \\
\hline AY-68-30-514 & $\mathrm{G}-5$ & -- & -- & 29.5786723125 & 98.3235744074 & 769 & 1925 \\
\hline AY-68-30-516 & U.C. no. 8 & -- & -- & 29.5499512247 & 98.3072346417 & 840 & 1980 \\
\hline AY-68-30-517 & Golden & -- & -- & 29.566944 & 98.332778 & 922 & 1977 \\
\hline AY-68-30-518 & U.C. no. 3 & -- & -- & 29.5527291513 & 98.3011234855 & 838 & 1982 \\
\hline AY-68-30-519 & $\mathrm{G}-43$ & -- & -- & 29.5530071428 & 98.3008464787 & 831 & 1950 \\
\hline AY-68-30-520 & Selma & -- & -- & 29.578056 & 98.331389 & 873 & 1988 \\
\hline AY-68-30-612 & G-10 & -- & -- & 29.5449057848 & 98.2868718677 & 762 & 1904 \\
\hline AY-68-30-616a & G-19 & -- & -- & 29.5421735431 & 98.2908461962 & 757 & 1929 \\
\hline${ }^{3} \mathrm{AY}-68-30-616 \mathrm{~b}$ & G-19 & -- & -- & 29.5397822294 & 98.2928044052 & 761 & 1929 \\
\hline AY-68-30-701 & $\mathrm{F}-246$ & -- & -- & 29.510508228 & 98.3408469988 & 769 & 1950 \\
\hline AY-68-30-702 & $\begin{array}{l}\text { Central Catholic High } \\
\text { School }\end{array}$ & -- & -- & 29.5057865576 & 98.3569588517 & 740 & 1966 \\
\hline AY-68-30-705 & Stonegate no. 1 & -- & -- & 29.5407853343 & 98.3672362023 & 920 & 1964 \\
\hline AY-68-30-708 & Don Sietma & -- & -- & 29.5157861996 & 98.3625140195 & 815 & 1970 \\
\hline AY-68-30-711 & TW-7 & -- & -- & 29.5132863504 & 98.3425134927 & 823 & 1979 \\
\hline AY-68-30-712 & BMWD no. 15 & -- & -- & 29.510556 & 98.357778 & 765 & 1977 \\
\hline AY-68-30-713 & Chapel Hill Memorial & -- & -- & 29.513333 & 98.339444 & 760 & 1976 \\
\hline
\end{tabular}




\begin{tabular}{|c|c|c|c|c|c|c|c|c|}
\hline $\begin{array}{l}\text { State well } \\
\text { number }\end{array}$ & $\begin{array}{l}\text { Well depth } \\
\text { (feet } \\
\text { below } \\
\text { LSD) }\end{array}$ & $\begin{array}{c}\text { Altitude of } \\
\text { top of Aus- } \\
\text { tin Group } \\
\text { (feet above } \\
\text { NAVD 88) }\end{array}$ & $\begin{array}{c}\text { Altitude of } \\
\text { base of Aus- } \\
\text { tin Group } \\
\text { (feet above } \\
\text { NAVD 88) }\end{array}$ & $\begin{array}{c}\text { Thickness } \\
\text { of Austin } \\
\text { Group } \\
\text { (feet) }\end{array}$ & Data source & Source agency & $\begin{array}{c}\text { Data } \\
\text { source date }\end{array}$ & $\begin{array}{c}\text { Report } \\
\text { author, } \\
\text { year }\end{array}$ \\
\hline AY-68-29-9kk & 312 & 678 & 556 & 122 & geophysical log & TWDB & $5 / 23 / 1986$ & unpublished data, 1986 \\
\hline AY-68-29-911 & 700 & 753 & 659 & 94 & geophysical log & TWDB & $10 / 23 / 1992$ & unpublished data, 1986 \\
\hline AY-68-29-9mm & 252 & 694 & 572 & 122 & geophysical log & TWDB & -- & unpublished data, -- \\
\hline AY-68-30-105 & 504 & 676 & 531 & 145 & drillers' log & TWDB & $8 / 23 / 1962$ & TWDB, 2009 \\
\hline AY-68-30-106 & 688 & 680 & 558 & 122 & drillers' log & TWDB & $3 / 6 / 1968$ & TWDB, 2009 \\
\hline AY-68-30-107 & 591 & 595 & 450 & 145 & drillers' log & TWDB & $12 / 1 / 1970$ & TWDB, 2009 \\
\hline AY-68-30-109 & 710 & 771 & 703 & 68 & geophysical log & USGS & $5 / 8 / 1973$ & unpublished data, 1973 \\
\hline AY-68-30-1aa & 270 & 805 & 648 & 157 & report/publication & TWDB & -- & Livingston, 1947 \\
\hline AY-68-30-1bb & 493 & 674 & 540 & 134 & geophysical log & TWDB & $10 / 18 / 1989$ & unpublished data, 1989 \\
\hline AY $-68-30-212$ & 481 & 771 & 511 & 260 & drillers' log & TWDB & 3/1/1968 & TWDB, 2009 \\
\hline AY-68-30-213 & 655 & 482 & 348 & 134 & drillers' log & TWDB & $9 / 20 / 1963$ & TWDB, 2009 \\
\hline AY $-68-30-214$ & 732 & 721 & 635 & 86 & drillers' log & TWDB & $1 / 31 / 1963$ & TWDB, 2009 \\
\hline AY-68-30-220 & 770 & 510 & 380 & 130 & drillers' log & TWDB & $6 / 20 / 1975$ & TWDB, 2009 \\
\hline AY-68-30-401 & 600 & 899 & 724 & 175 & drillers' log & $\begin{array}{c}\text { City of Live } \\
\text { Oak }\end{array}$ & $8 / 16 / 1965$ & unpublished data, 1965 \\
\hline AY-68-30-402 & 592 & 795 & 697 & 98 & drillers' log & TWDB & $6 / 17 / 1969$ & Petitt and George, 1956 \\
\hline AY $-68-30-403$ & 650 & 758 & 634 & 124 & geophysical log & $\begin{array}{c}\text { City of Live } \\
\text { Oak }\end{array}$ & $5 / 30 / 1972$ & unpublished data, 1972 \\
\hline AY-68-30-404 & 509 & 842 & 716 & 126 & drillers' log & TWDB & $12 / 1 / 1939$ & TWDB, 2009 \\
\hline AY $-68-30-405$ & 802 & 681 & 590 & 91 & geophysical log & USGS & $1 / 15 / 1973$ & unpublished data, 1973 \\
\hline AY-68-30-406 & 390 & 748 & 646 & 102 & drillers' log & TWDB & $5 / 18 / 1967$ & TWDB, 2009 \\
\hline $\mathrm{AY}-68-30-4 \mathrm{bb}$ & 547 & 880 & 715 & 165 & geophysical log & TWDB & $11 / 5 / 1992$ & unpublished data, 1992 \\
\hline AY-68-30-506 & 585 & 713 & 583 & 130 & drillers' log & TWDB & 8/1/1967 & TWDB, 2009 \\
\hline AY-68-30-510 & 425 & 689 & 556 & 133 & drillers' log & City of Schertz & $5 / 5 / 1905$ & unpublished data, 1905 \\
\hline AY-68-30-511 & 630 & 595 & 422 & 173 & drillers' log & TWDB & $4 / 25 / 1963$ & TWDB, 2009 \\
\hline AY $-68-30-512$ & 636 & 810 & 680 & 130 & geophysical log & $\begin{array}{l}\text { City of Univer- } \\
\text { sal City }\end{array}$ & $1 / 13 / 1972$ & unpublished data, 1972 \\
\hline AY-68-30-514 & 1,497 & 769 & 627 & 142 & report/publication & TWDB & -- & Livingston, 1947 \\
\hline AY-68-30-516 & 720 & 715 & 616 & 99 & drillers' log & $\begin{array}{c}\text { City of Univer- } \\
\text { sal City }\end{array}$ & $12 / 31 / 1980$ & unpublished data, 1980 \\
\hline AY-68-30-517 & 520 & 922 & 652 & 270 & drillers' log & TWDB & 9/9/1977 & TWDB, 2009 \\
\hline AY-68-30-518 & 520 & 764 & 581 & 183 & drillers' log & TWDB & $7 / 26 / 1982$ & TWDB, 2009 \\
\hline AY-68-30-519 & 426 & 701 & 572 & 129 & drillers' log & TWDB & $3 / 15 / 1950$ & TWDB, 2009 \\
\hline AY-68-30-520 & 777 & 725 & 583 & 142 & drillers' log & EAA & $11 / 7 / 1988$ & unpublished data, 1988 \\
\hline AY-68-30-612 & 554 & 474 & 394 & 80 & report/publication & TWDB & -- & Livingston, 1947 \\
\hline AY-68-30-616a & 1,003 & 627 & 422 & 205 & geophysical log & USGS & $5 / 29 / 1973$ & Petitt and George, 1956 \\
\hline${ }^{3} A Y-68-30-616 b$ & 1,003 & 596 & 411 & 185 & geophysical log & TWDB & -- & Livingston, 1947 \\
\hline AY-68-30-701 & 645 & 487 & 348 & 139 & drillers' log & TWDB & 9/10/1958 & TWDB, 2009 \\
\hline AY-68-30-702 & 589 & 493 & 360 & 133 & drillers' log & TWDB & $5 / 1 / 1966$ & TWDB, 2009 \\
\hline AY-68-30-705 & 745 & 824 & 710 & 114 & geophysical log & USGS & $10 / 14 / 1964$ & unpublished data, 1964 \\
\hline AY-68-30-708 & 938 & 725 & 471 & 254 & geophysical log & USGS & $10 / 29 / 1971$ & unpublished data, 1971 \\
\hline AY-68-30-711 & -- & 593 & 459 & 134 & geophysical log & USGS & $10 / 10 / 1979$ & unpublished data, 1979 \\
\hline AY-68-30-712 & 837 & 550 & 429 & 121 & drillers' log & TWDB & $5 / 26 / 1977$ & TWDB, 2009 \\
\hline AY-68-30-713 & 675 & 525 & 360 & 165 & drillers' log & TWDB & 9/1/1976 & TWDB, 2009 \\
\hline
\end{tabular}


Appendix 1. Wells selected for inclusion in geodatabase of selected wells penetrating the Austin Group, central Bexar County, Texas,

\begin{tabular}{|c|c|c|c|c|c|c|c|}
\hline AY-68-30-714 & Converse no. 2 & -- & -- & 29.536111 & 98.336111 & 820 & 1987 \\
\hline AY-68-30-715 & Converse no. 9 & -- & -- & 29.53578561 & 98.3355693592 & 815 & 1985 \\
\hline AY-68-30-718 & Lackland no. 18 & 038 WP no. 1 & -- & 29.530278 & 98.353611 & 890 & 1984 \\
\hline AY-68-30-7bb & $\mathrm{F}-82$ & -- & -- & 29.5382432401 & 98.3469841019 & 834 & 1939 \\
\hline AY-68-30-7cc & N. Cimmaron no. 2 & -- & -- & 29.5361111111 & 98.3352777778 & 815 & -- \\
\hline AY-68-30-7dd & Mel Murray & -- & -- & 29.5291186364 & 98.362236488 & 905 & 1978 \\
\hline AY-68-30-8aa & G-16 & -- & -- & 29.5378946036 & 98.2944223701 & 762 & 1929 \\
\hline AY-68-30-8bb & $\mathrm{G}-17$ & -- & -- & 29.539242907 & 98.2937482181 & 762 & 1929 \\
\hline AY-68-30-8dd & $\mathrm{G}-20$ & -- & -- & 29.5365462991 & 98.2959055047 & 762 & 1942 \\
\hline AY-68-30-8ee & U.C. no. 8 & -- & -- & 29.5299520615 & 98.3261244328 & 765 & -- \\
\hline AY-68-30-9aа & G-11 & -- & -- & 29.5407260417 & 98.2891639842 & 755 & 1928 \\
\hline AY-68-30-9bb & $\mathrm{G}-12$ & -- & -- & 29.5388384163 & 98.2917257625 & 763 & 1929 \\
\hline AY-68-30-9cc & $\mathrm{G}-13$ & -- & -- & 29.5409957023 & 98.2917257617 & 761 & 1929 \\
\hline AY-68-30-9dd & $\mathrm{G}-14$ & -- & -- & 29.5392429076 & 98.2902426279 & 761 & 1929 \\
\hline AY-68-30-9ee & $\mathrm{G}-15$ & -- & -- & 29.5403215511 & 98.2906471188 & 760 & 1929 \\
\hline AY-68-30-9ff & $\mathrm{G}-18$ & -- & -- & 29.5418046854 & 98.2875460193 & 755 & 1929 \\
\hline AY-68-34-3hh & 32579 & -- & -- & 29.474722 & 98.758611 & 922 & -- \\
\hline AY-68-34-3jj & 78280 & -- & -- & 29.478056 & 98.759167 & 949 & -- \\
\hline AY-68-34-601 & BMWD-Elm Valley & -- & -- & 29.45773206 & 98.763634976 & 892 & 1964 \\
\hline AY-68-34-604 & Tejas Valley RV Park & -- & -- & 29.4257889888 & 98.7539126049 & 893 & 1989 \\
\hline AY-68-34-6aа & 31287 & -- & -- & 29.432778 & 98.759444 & 912 & -- \\
\hline AY-68-34-6bb & 40281 & -- & -- & 29.433333 & 98.766389 & 887 & -- \\
\hline AY-68-34-6cc & 88101 & -- & -- & 29.432222 & 98.756389 & 912 & -- \\
\hline AY-68-34-6dd & 84684 & -- & -- & 29.431667 & 98.763333 & 866 & -- \\
\hline AY-68-34-6ee & 33235 & -- & -- & 29.4275 & 98.757222 & 894 & -- \\
\hline AY-68-34-6ff & 37990 & -- & -- & 29.426944 & 98.7575 & 870 & -- \\
\hline AY-68-34-6gg & 85728 & -- & -- & 29.425 & 98.776944 & 962 & -- \\
\hline AY-68-34-6hh & 28776 & -- & -- & 29.423333 & 98.756944 & 857 & -- \\
\hline AY-68-34-611 & 49312 & -- & -- & 29.445 & 98.767222 & 912 & -- \\
\hline AY-68-34-6mm & Palayo & -- & -- & 29.4307883974 & 98.7561350183 & 890 & -- \\
\hline AY-68-34-802 & Texas Research Park no. 1 & 039 WP no. 1 & -- & 29.4119003277 & 98.7961358808 & 900 & 1989 \\
\hline AY-68-34-803 & Texas Research Park no. 2 & -- & -- & 29.4118999944 & 98.7961362142 & 904 & -- \\
\hline AY-68-34-8cc & Pioneer Estates & -- & -- & 29.3769009747 & 98.7958583588 & 795 & -- \\
\hline
\end{tabular}




\begin{tabular}{|c|c|c|c|c|c|c|c|c|}
\hline $\begin{array}{l}\text { State well } \\
\text { number }\end{array}$ & $\begin{array}{l}\text { Well depth } \\
\text { (feet } \\
\text { below } \\
\text { LSD) }\end{array}$ & $\begin{array}{l}\text { Altitude of } \\
\text { top of Aus- } \\
\text { tin Group } \\
\text { (feet above } \\
\text { NAVD 88) }\end{array}$ & $\begin{array}{c}\text { Altitude of } \\
\text { base of Aus- } \\
\text { tin Group } \\
\text { (feet above } \\
\text { NAVD 88) }\end{array}$ & $\begin{array}{c}\text { Thickness } \\
\text { of Austin } \\
\text { Group } \\
\text { (feet) }\end{array}$ & Data source & Source agency & $\begin{array}{c}\text { Data } \\
\text { source date }\end{array}$ & $\begin{array}{c}\text { Report } \\
\text { author, } \\
\text { year }^{1}\end{array}$ \\
\hline AY-68-30-714 & 650 & 590 & 464 & 126 & drillers' log & TWDB & $5 / 27 / 1987$ & TWDB, 2009 \\
\hline AY-68-30-715 & 690 & 585 & 450 & 135 & drillers' log & TWDB & $7 / 22 / 1985$ & TWDB, 2009 \\
\hline AY-68-30-716 & 764 & 599 & 484 & 115 & drillers' log & TWDB & $4 / 20 / 1990$ & TWDB, 2009 \\
\hline AY $-68-30-717$ & 861 & 577 & 352 & 225 & drillers' log & TWDB & $2 / 18 / 1993$ & TWDB, 2009 \\
\hline AY-68-30-718 & 874 & 742 & 482 & 260 & drillers' log & BMWD & $12 / 27 / 1984$ & unpublished data, 1984 \\
\hline AY-68-30-7bb & 417 & 716 & 584 & 132 & report/publication & TWDB & -- & Livingston, 1947 \\
\hline AY-68-30-7cc & 653 & 585 & 461 & 124 & geophysical log & TWDB & $6 / 15 / 1987$ & unpublished data, 1987 \\
\hline AY-68-30-7dd & 630 & 685 & 519 & 166 & geophysical log & TWDB & $6 / 24 / 1979$ & unpublished data, 1979 \\
\hline AY-68-30-802 & 750 & 340 & 230 & 110 & drillers' log & TWDB & $8 / 1 / 1954$ & unpublished data, 1954 \\
\hline AY-68-30-807 & 1,202 & 498 & 288 & 210 & geophysical log & USGS & $11 / 21 / 1972$ & unpublished data, 1972 \\
\hline AY-68-30-810 & 760 & 565 & 345 & 220 & geophysical log & TWDB & $11 / 3 / 1952$ & TWDB, 2009 \\
\hline AY-68-30-8aа & 609 & 537 & 412 & 125 & report/publication & TWDB & -- & Livingston, 1947 \\
\hline AY-68-30-8bb & 583 & 530 & 412 & 118 & report/publication & TWDB & -- & Livingston, 1947 \\
\hline AY-68-30-8dd & 518 & 562 & 412 & 150 & report/publication & TWDB & -- & Livingston, 1947 \\
\hline AY-68-30-8ee & 420 & 725 & 540 & 185 & geophysical log & TWDB & $7 / 2 / 1980$ & unpublished data, 1980 \\
\hline AY-68-30-9aа & 700 & 569 & 412 & 157 & report/publication & TWDB & -- & Livingston, 1947 \\
\hline AY-68-30-9bb & 563 & 552 & 418 & 134 & report/publication & TWDB & -- & Livingston, 1947 \\
\hline AY-68-30-9cc & 584 & 539 & 417 & 122 & report/publication & TWDB & -- & Livingston, 1947 \\
\hline AY-68-30-9dd & 584 & 537 & 417 & 120 & report/publication & TWDB & -- & Livingston, 1947 \\
\hline AY-68-30-9ee & 584 & 540 & 418 & 122 & report/publication & TWDB & -- & Livingston, 1947 \\
\hline AY-68-30-9ff & 577 & 583 & 411 & 172 & geophysical log & TWDB & -- & Livingston, 1947 \\
\hline AY-68-30-9gg & 750 & 265 & 155 & 110 & geophysical log & TWDB & 2/4/1982 & unpublished data, 1982 \\
\hline AY-68-34-203 & 1,000 & 537 & 319 & 218 & drillers' log & BMWD & $9 / 23 / 1969$ & unpublished data, 1969 \\
\hline AY-68-34-3dd & -- & 881 & 633 & 248 & drillers' log & TWDB & $8 / 1 / 2002$ & unpublished data, 2002 \\
\hline AY-68-34-3ee & -- & 814 & 614 & 200 & drillers' log & TWDB & $7 / 8 / 2003$ & unpublished data, 2003 \\
\hline AY-68-34-3ff & -- & 954 & 814 & 140 & drillers' log & TWDB & $6 / 11 / 2003$ & unpublished data, 2003 \\
\hline AY-68-34-3gg & -- & 878 & 627 & 251 & drillers' log & TWDB & $1 / 16 / 2003$ & unpublished data, 2003 \\
\hline AY-68-34-3hh & -- & 922 & 837 & 85 & drillers' log & TWDB & $12 / 26 / 2002$ & unpublished data, 2002 \\
\hline AY-68-34-3jj & -- & 949 & 829 & 120 & drillers' log & TWDB & $3 / 6 / 2006$ & unpublished data, 2006 \\
\hline AY-68-34-601 & 395 & 892 & 841 & 51 & drillers' log & BMWD & $5 / 1 / 1964$ & unpublished data, 1964 \\
\hline AY-68-34-604 & 664 & 658 & 533 & 125 & drillers' log & TWDB & $4 / 12 / 1989$ & TWDB, 2009 \\
\hline AY-68-34-6aа & -- & 912 & 752 & 160 & drillers' log & TWDB & $12 / 2 / 2003$ & unpublished data, 2003 \\
\hline AY-68-34-6bb & -- & 887 & 747 & 140 & drillers' log & TWDB & $6 / 30 / 2004$ & unpublished data, 2004 \\
\hline AY-68-34-6cc & -- & 912 & 742 & 170 & drillers' log & TWDB & $7 / 18 / 2006$ & unpublished data, 2006 \\
\hline AY-68-34-6dd & -- & 866 & 741 & 125 & drillers' log & TWDB & $4 / 7 / 2004$ & unpublished data, 2004 \\
\hline AY-68-34-6ee & -- & 894 & 734 & 160 & drillers' log & TWDB & $2 / 23 / 2004$ & unpublished data, 2004 \\
\hline AY-68-34-6ff & -- & 870 & 720 & 150 & drillers' log & TWDB & $5 / 7 / 2004$ & unpublished data, 2004 \\
\hline AY-68-34-6gg & -- & 912 & 727 & 185 & drillers' log & TWDB & $6 / 8 / 2006$ & unpublished data, 2006 \\
\hline AY-68-34-6hh & -- & 857 & 747 & 110 & drillers' log & TWDB & $10 / 23 / 2003$ & unpublished data, 2003 \\
\hline AY-68-34-611 & -- & 912 & 762 & 150 & drillers' log & TWDB & $11 / 10 / 2004$ & unpublished data, 2004 \\
\hline AY-68-34-6mm & 459 & 890 & 758 & 132 & geophysical log & TWDB & 9/30/1991 & unpublished data, 1991 \\
\hline AY-68-34-802 & 677 & 868 & 678 & 190 & geophysical log & EAA & $5 / 22 / 1989$ & unpublished data, 1989 \\
\hline AY-68-34-803 & 667 & 874 & 684 & 190 & geophysical log & TWDB & 6/7/1989 & TWDB, 2009 \\
\hline AY-68-34-8cc & 1,140 & 337 & 145 & 192 & geophysical log & TWDB & $2 / 10 / 1992$ & unpublished data, 2009 \\
\hline
\end{tabular}


Appendix 1. Wells selected for inclusion in geodatabase of selected wells penetrating the Austin Group, central Bexar County, Texas,

\begin{tabular}{|c|c|c|c|c|c|c|c|}
\hline $\begin{array}{l}\text { State well } \\
\text { number }\end{array}$ & $\begin{array}{l}\text { First alternate } \\
\text { well identifier }\end{array}$ & $\begin{array}{c}\text { Second } \\
\text { alternate } \\
\text { well } \\
\text { identifier }\end{array}$ & $\begin{array}{l}\text { Third } \\
\text { alternate } \\
\text { well } \\
\text { identifier }\end{array}$ & $\begin{array}{l}\text { Latitude } \\
\text { (dd) }\end{array}$ & $\begin{array}{l}\text { Longitude } \\
\text { (dd) }\end{array}$ & $\begin{array}{l}\text { Land-surface } \\
\text { altitude } \\
\text { (feet above } \\
\text { NAVD 88) }\end{array}$ & $\begin{array}{l}\text { Year } \\
\text { drilled }\end{array}$ \\
\hline AY-68-34-8dd & Texas Research Park no. 3 & -- & -- & 29.4132888318 & 98.8047475947 & 975 & -- \\
\hline AY-68-34-901 & Briggs Ranch no. 2 & -- & -- & 29.3902339737 & 98.7761352153 & 793 & 1957 \\
\hline AY-68-34-905 & Briggs no. 8 & -- & -- & 29.3796782677 & 98.7789132796 & 752 & 1967 \\
\hline AY-68-34-906 & Briggs no. 1 & $\mathrm{H}-73$ & -- & 29.389678 & 98.7708580497 & 785 & 1942 \\
\hline AY-68-34-907 & Briggs no. 6 & -- & -- & 29.3896780331 & 98.7541905306 & 759 & 1966 \\
\hline AY-68-35-101 & Anderson no. 3 & 083 WP no. 1 & -- & 29.4777314285 & 98.7150227382 & 1,002 & 1973 \\
\hline AY-68-35-102 & Anderson no. 1 & -- & -- & 29.4791203813 & 98.7116896596 & 982 & 1976 \\
\hline AY-68-35-1cc & F.D.I.C. & -- & -- & 29.4646762659 & 98.7166896305 & 910 & -- \\
\hline AY-68-35-1dd & Emilio Garza & -- & -- & 29.4932861032 & 98.4933508856 & 1,090 & -- \\
\hline AY-68-35-203 & Sea World no. 1 & -- & -- & 29.4630098532 & 98.7022443743 & 1,000 & 1985 \\
\hline AY-68-35-2cc & Langford & -- & -- & 29.4949531155 & 98.7055783349 & 850 & -- \\
\hline AY-68-35-2dd & Laredo Warehouse & -- & -- & 29.4863888889 & 98.6719444444 & 824 & -- \\
\hline AY-68-35-307 & $1185-\mathrm{LG}$ & -- & -- & 29.46467615 & 98.6269651597 & 770 & -- \\
\hline AY-68-35-308 & Royal Crest & -- & -- & 29.4627317845 & 98.6400210342 & 818 & 1972 \\
\hline AY-68-35-309 & Allied Concrete & -- & -- & 29.4671756239 & 98.6261314833 & 800 & 1972 \\
\hline AY-68-35-310 & Timber Creek no. 2 & -- & -- & 29.4905088782 & 98.6297426768 & 850 & 1971 \\
\hline AY-68-35-3aа & Sawyer & -- & -- & 29.49 & 98.6263888889 & 867 & -- \\
\hline AY-68-35-404 & Zedler & -- & -- & 29.4441214612 & 98.7503015361 & 860 & 1967 \\
\hline AY-68-35-405 & Quest Development no. 1 & -- & -- & 29.437778 & 98.721389 & 850 & 1987 \\
\hline AY-68-35-4dd & Quest Development no. 2 & -- & -- & 29.4349549334 & 98.7191896079 & 833 & -- \\
\hline AY-68-35-4ee & Denton Development & -- & -- & 29.4380103937 & 98.712244995 & 830 & -- \\
\hline AY-68-35-505 & Ray Ellison at Heritage & -- & -- & 29.4316217982 & 98.6900219618 & 890 & 1983 \\
\hline AY-68-35-506 & Sea World no. 2 & -- & -- & 29.4532881467 & 98.7014113225 & 990 & 1986 \\
\hline AY-68-35-507 & Sea World no. 3 & -- & -- & 29.456065057 & 98.6961331983 & 970 & 1987 \\
\hline AY-68-35-601 & SWRI no. 2 & -- & -- & 29.4371765992 & 98.6391876823 & 760 & 1966 \\
\hline AY-68-35-603 & Buch & -- & -- & 29.4310657957 & 98.6427987489 & 735 & 1956 \\
\hline AY-68-35-604 & Saunders & -- & -- & 29.4310657957 & 98.6427987489 & 750 & 1958 \\
\hline AY-68-35-607 & Southwest Utility Co. & -- & -- & 29.4230110492 & 98.6286313498 & 724 & 1967 \\
\hline AY-68-35-608 & 0195-LG & -- & -- & 29.4182892026 & 98.6455767672 & 752 & 1956 \\
\hline AY-68-35-610 & $\mathrm{I}-6$ & -- & -- & 29.4510651592 & 98.6427988327 & 789 & 1940 \\
\hline AY-68-35-613 & Tom Slick & $\mathrm{I}-4$ & -- & 29.4446763747 & 98.6586322082 & 782 & 1940 \\
\hline AY-68-35-614 & Westlakes no. 1 & -- & -- & 29.4235660381 & 98.6600211613 & 774 & 1985 \\
\hline AY-68-35-615 & Westlakes no. 2 & -- & -- & 29.4291218602 & 98.6500209262 & 761 & 1985 \\
\hline
\end{tabular}


2010-Continued.

\begin{tabular}{|c|c|c|c|c|c|c|c|c|}
\hline $\begin{array}{l}\text { State well } \\
\text { number }\end{array}$ & $\begin{array}{l}\text { Well depth } \\
\text { (feet } \\
\text { below } \\
\text { LSD) }\end{array}$ & $\begin{array}{c}\text { Altitude of } \\
\text { top of Aus- } \\
\text { tin Group } \\
\text { (feet above } \\
\text { NAVD 88) }\end{array}$ & $\begin{array}{c}\text { Altitude of } \\
\text { base of Aus- } \\
\text { tin Group } \\
\text { (feet above } \\
\text { NAVD 88) }\end{array}$ & $\begin{array}{c}\text { Thickness } \\
\text { of Austin } \\
\text { Group } \\
\text { (feet) }\end{array}$ & Data source & Source agency & $\begin{array}{c}\text { Data } \\
\text { source date }\end{array}$ & $\begin{array}{c}\text { Report } \\
\text { author, } \\
\text { year' }^{1}\end{array}$ \\
\hline AY-68-34-8dd & 463 & 898 & 712 & 186 & geophysical log & TWDB & $12 / 1 / 1987$ & unpublished data, 1987 \\
\hline AY-68-34-901 & 827 & 463 & 319 & 144 & geophysical log & EAA & $12 / 20 / 1989$ & unpublished data, 1989 \\
\hline AY-68-34-903 & 851 & 541 & 153 & 388 & drillers' log & TWDB & 9/1/1964 & TWDB, 2009 \\
\hline AY-68-34-904 & 1,142 & 437 & 211 & 226 & drillers' log & TWDB & $8 / 29 / 1964$ & TWDB, 2009 \\
\hline AY-68-34-905 & 1,015 & 429 & 235 & 194 & drillers' log & TWDB & $9 / 29 / 1967$ & TWDB, 2009 \\
\hline AY-68-34-906 & 905 & 485 & 145 & 340 & drillers' log & TWDB & $7 / 24 / 1951$ & Petitt and George, 1956 \\
\hline AY-68-34-907 & 1,143 & 437 & 254 & 183 & drillers' log & TWDB & $12 / 1 / 1966$ & TWDB, 2009 \\
\hline AY-68-35-101 & 572 & 1,002 & 902 & 100 & geophysical log & SAWS & $5 / 6 / 1985$ & unpublished data, 1985 \\
\hline AY-68-35-102 & 796 & 982 & 872 & 110 & geophysical log & TWDB & $11 / 21 / 1975$ & TWDB, 2009 \\
\hline AY-68-35-103 & 810 & 993 & 873 & 120 & drillers' log & SAWS & $6 / 8 / 1979$ & unpublished data, 1979 \\
\hline AY-68-35-105 & 750 & 962 & 842 & 120 & drillers' log & SAWS & $2 / 25 / 1986$ & unpublished data, 1986 \\
\hline AY-68-35-106 & 760 & 955 & 835 & 120 & drillers' log & SAWS & 4/1/1986 & unpublished data, 1986 \\
\hline AY-68-35-1cc & 315 & 910 & 820 & 90 & geophysical log & TWDB & $10 / 27 / 1992$ & unpublished data, 1992 \\
\hline AY-68-35-1dd & 546 & 888 & 731 & 157 & geophysical log & TWDB & 3/3/1988 & unpublished data, 1988 \\
\hline AY-68-35-203 & 540 & 937 & 776 & 161 & drillers' log & TWDB & $12 / 17 / 1985$ & TWDB, 2009 \\
\hline $\mathrm{AY}-68-35-2 \mathrm{cc}$ & 353 & 850 & 722 & 128 & geophysical log & TWDB & $2 / 21 / 1978$ & unpublished data, 1978 \\
\hline AY-68-35-2dd & 235 & 824 & 765 & 59 & geophysical log & TWDB & $3 / 5 / 2005$ & unpublished data, 2005 \\
\hline AY-68-35-307 & 225 & 770 & 674 & 96 & geophysical log & TWDB & $11 / 5 / 1985$ & TWDB, 2009 \\
\hline AY-68-35-308 & 607 & 818 & 683 & 135 & geophysical log & TWDB & $3 / 29 / 1972$ & TWDB, 2009 \\
\hline AY-68-35-309 & 441 & 800 & 665 & 135 & drillers' log & TWDB & $7 / 1 / 1975$ & TWDB, 2009 \\
\hline AY-68-35-310 & 427 & 850 & 704 & 146 & drillers' log & USGS & $2 / 10 / 1971$ & unpublished data, 1971 \\
\hline AY-68-35-3aа & 397 & 763 & 591 & 172 & geophysical log & TWDB & $3 / 6 / 2005$ & unpublished data, 2005 \\
\hline AY-68-35-3bb & 385 & 820 & 663 & 157 & geophysical log & TWDB & $12 / 2 / 1999$ & unpublished data, 1999 \\
\hline AY $-68-35-3 c c$ & 399 & 735 & 569 & 166 & geophysical log & TWDB & $5 / 5 / 1999$ & unpublished data, 1999 \\
\hline AY-68-35-3dd & 407 & 855 & 711 & 144 & geophysical log & TWDB & 4/3/1992 & unpublished data, 1992 \\
\hline AY-68-35-3ee & 399 & 735 & 570 & 165 & geophysical log & TWDB & $5 / 5 / 1999$ & unpublished data, 1999 \\
\hline AY-68-35-401 & 301 & 862 & 796 & 66 & geophysical log & USGS & $6 / 12 / 1972$ & unpublished data, 1972 \\
\hline AY-68-35-402 & 606 & 767 & 562 & 205 & drillers' log & TWDB & $1 / 26 / 1972$ & TWDB, 2009 \\
\hline AY-68-35-404 & 314 & 860 & 775 & 85 & drillers' log & TWDB & $4 / 19 / 1967$ & TWDB, 2009 \\
\hline AY-68-35-405 & 405 & 850 & 715 & 135 & drillers' log & TWDB & $4 / 13 / 1987$ & TWDB, 2009 \\
\hline AY-68-35-4dd & 407 & 707 & 526 & 181 & geophysical log & TWDB & $2 / 18 / 1986$ & unpublished data, 1986 \\
\hline AY-68-35-4ee & 368 & 744 & 576 & 168 & geophysical log & TWDB & $5 / 22 / 1995$ & unpublished data, 1995 \\
\hline AY-68-35-505 & 900 & 771 & 605 & 166 & geophysical log & TWDB & $6 / 1 / 1983$ & TWDB, 2009 \\
\hline AY-68-35-506 & 700 & 990 & 843 & 147 & drillers' log & TWDB & $11 / 22 / 1986$ & TWDB, 2009 \\
\hline AY-68-35-507 & 700 & 935 & 785 & 150 & drillers' log & TWDB & $1 / 13 / 1987$ & TWDB, 2009 \\
\hline AY-68-35-601 & 900 & 556 & 402 & 154 & geophysical log & TWDB & $5 / 18 / 1966$ & TWDB, 2009 \\
\hline AY-68-35-603 & 618 & 540 & 370 & 170 & drillers' log & TWDB & $6 / 1 / 1956$ & TWDB, 2009 \\
\hline AY-68-35-604 & 906 & 542 & 386 & 156 & drillers' log & TWDB & $4 / 19 / 1958$ & TWDB, 2009 \\
\hline AY-68-35-607 & 701 & 491 & 334 & 157 & drillers' log & TWDB & $7 / 1 / 1967$ & TWDB, 2009 \\
\hline AY-68-35-608 & 638 & 489 & 332 & 157 & geophysical log & USGS & $1 / 26 / 1994$ & unpublished data, 1994 \\
\hline AY-68-35-610 & 763 & 651 & 493 & 158 & drillers' log & TWDB & $1 / 1 / 1940$ & Petitt and George, 1956 \\
\hline AY-68-35-613 & 545 & 706 & 538 & 168 & geophysical log & EAA & $5 / 18 / 1993$ & Petitt and George, 1956 \\
\hline AY-68-35-614 & 760 & 579 & 324 & 255 & drillers' log & TWDB & $6 / 25 / 1985$ & TWDB, 2009 \\
\hline AY-68-35-615 & 760 & 543 & 346 & 197 & drillers' log & TWDB & 7/9/1985 & TWDB, 2009 \\
\hline
\end{tabular}


Appendix 1. Wells selected for inclusion in geodatabase of selected wells penetrating the Austin Group, central Bexar County, Texas,

\begin{tabular}{|c|c|c|c|c|c|c|c|}
\hline $\begin{array}{c}\text { State well } \\
\text { number }\end{array}$ & $\begin{array}{l}\text { First alternate } \\
\text { well identifier }\end{array}$ & $\begin{array}{c}\text { Second } \\
\text { alternate } \\
\text { well } \\
\text { identifier }\end{array}$ & $\begin{array}{c}\text { Third } \\
\text { alternate } \\
\text { well } \\
\text { identifier }\end{array}$ & $\begin{array}{l}\text { Latitude } \\
\text { (dd) }\end{array}$ & $\begin{array}{l}\text { Longitude } \\
\text { (dd) }\end{array}$ & $\begin{array}{l}\text { Land-surface } \\
\text { altitude } \\
\text { (feet above } \\
\text { NAVD 88) }\end{array}$ & $\begin{array}{l}\text { Year } \\
\text { drilled }\end{array}$ \\
\hline AY-68-35-616 & Micron no. 1 & -- & -- & 29.4549540343 & 98.641687821 & 817 & 2002 \\
\hline AY-68-35-617 & Micron no. 2 & -- & -- & 29.4543990517 & 98.6414098117 & 816 & 2002 \\
\hline AY-68-35-6dd & $\mathrm{I}-48$ & -- & -- & 29.4361449687 & 98.6271386687 & 729 & 1942 \\
\hline AY-68-35-6еe & I-49 & -- & -- & 29.421646233 & 98.6338051331 & 745 & 1931 \\
\hline AY-68-35-6ff & R.T.C. & -- & -- & 29.4563430913 & 98.632520818 & 750 & -- \\
\hline AY-68-35-6gg & Leeper & -- & -- & 29.4530098732 & 98.6408543473 & 810 & -- \\
\hline AY-68-35-705 & Briggs no. 7 & -- & -- & 29.3838452185 & 98.7400231239 & 765 & 1966 \\
\hline AY-68-35-710 & Air Force Village no. 1 & -- & -- & 29.3860671528 & 98.7408571515 & 761 & 1985 \\
\hline AY-68-35-711 & Air Force Village no. 4 & -- & -- & 29.3855121693 & 98.7405791427 & 763 & 1985 \\
\hline AY-68-35-712 & Benke & -- & -- & 29.396900843 & 98.7258567849 & 780 & -- \\
\hline AY-68-35-713 & Air Force Village no. 2 & -- & -- & 29.387178123 & 98.7372450589 & 772 & 1991 \\
\hline AY-68-35-7bb & Air Force Village no. 3 & -- & -- & 29.385833 & 98.738056 & 768 & -- \\
\hline AY-68-35-7ee & 55447 & -- & -- & 29.381944 & 98.728611 & 715 & -- \\
\hline AY-68-35-7ff & 77106 & -- & -- & 29.406944 & 98.746667 & 865 & -- \\
\hline AY-68-35-7gg & Harlach & -- & -- & 29.3816232031 & 98.705300198 & 695 & -- \\
\hline AY-68-35-7hh & Jungman & -- & -- & 29.3830120277 & 98.73168979 & 704 & -- \\
\hline AY-68-35-801 & $\mathrm{H}-102$ & -- & -- & 29.4069005537 & 98.693077958 & 768 & 1957 \\
\hline AY-68-35-808 & Adams Hill & -- & -- & 29.4085665056 & 98.678077573 & 730 & 1972 \\
\hline AY-68-35-810 & Uptmore Development & $17 \mathrm{~A}$ & -- & 29.4066225597 & 98.7041892468 & 835 & 1981 \\
\hline AY-68-35-811 & Uptmore Development & 17B & -- & 29.4069005512 & 98.7044672549 & 835 & 1981 \\
\hline AY-68-35-8bb & West side Airpark & -- & -- & 29.3941228394 & 98.6805773648 & 732 & -- \\
\hline AY-68-35-903 & $\mathrm{I}-264$ & -- & -- & 29.4085668472 & 98.6311316841 & 740 & 1961 \\
\hline AY-68-35-904 & W.C.I.D. no. 16 & Well no. 2 & -- & 29.4063445794 & 98.6561319929 & 789 & 1958 \\
\hline AY-68-35-905 & $\mathrm{I}-231$ & -- & -- & 29.4044003112 & 98.642243067 & 760 & 1957 \\
\hline AY-68-35-910 & W.C.I.D. no. 16 & -- & -- & 29.4096774744 & 98.6569660278 & 781 & 1967 \\
\hline AY-68-35-912 & CWB Marbach no. 1 & -- & -- & 29.4166222553 & 98.6402986236 & 757 & 1973 \\
\hline AY-68-35-913 & CWB Marbach no. 2 & -- & -- & 29.4163442642 & 98.6394656008 & 757 & 1974 \\
\hline AY-68-36-100 & Green Tree & -- & -- & 29.4775 & 98.599444 & 821 & -- \\
\hline AY-68-36-102 & CWB Wurzbach no. 1 & -- & -- & 29.4896748488 & 98.6008539616 & 901 & 1963 \\
\hline AY-68-36-103 & Wurzbach no. 2 & -- & -- & 29.4888419889 & 98.601686867 & 910 & 1967 \\
\hline AY-68-36-104 & Wurzbach no. 3 & -- & -- & 29.4905085991 & 98.6008535213 & 888 & 1968 \\
\hline AY-68-36-107 & Grass Hill & -- & -- & 29.4885639035 & 98.6102981889 & 882 & 1970 \\
\hline AY-68-36-108 & Craig & -- & -- & 29.479398176 & 98.5941867457 & 870 & 1963 \\
\hline AY-68-36-111 & Timber Creek & -- & -- & 29.4752313557 & 98.6208543881 & 802 & 1972 \\
\hline AY-68-36-121 & I-174 & -- & -- & 29.4960636801 & 98.6205764766 & 825 & 1950 \\
\hline AY-68-36-124 & Dean & -- & -- & 29.496063676 & 98.6186314289 & 825 & 1969 \\
\hline AY-68-36-129 & Lewis & -- & -- & 29.4874529481 & 98.6147422933 & 840 & 1972 \\
\hline${ }^{2} \mathrm{AY}-68-36-134$ & -- & -- & -- & 29.495277 & 98.595555 & 841 & 2007 \\
\hline AY-68-36-1aa & Mursch & -- & -- & 29.484722 & 98.606111 & 900 & -- \\
\hline AY-68-36-1bb & Worth & -- & -- & 29.481667 & 98.607778 & 888 & -- \\
\hline AY-68-36-1cc & Evers and Assoc. & -- & -- & 29.482222 & 98.591111 & 864 & -- \\
\hline AY-68-36-1dd & I-39 & -- & -- & 29.4649685544 & 98.5867430266 & 903 & -- \\
\hline AY-68-36-1ee & $4 \mathrm{M}$ properties & -- & -- & 29.4669444444 & 98.6058333333 & 880 & -- \\
\hline AY-68-36-1ff & Theo & -- & -- & 29.4830088524 & 98.6044646848 & 885 & -- \\
\hline
\end{tabular}




\begin{tabular}{|c|c|c|c|c|c|c|c|c|}
\hline $\begin{array}{c}\text { State well } \\
\text { number }\end{array}$ & $\begin{array}{l}\text { Well depth } \\
\text { (feet } \\
\text { below } \\
\text { LSD) }\end{array}$ & $\begin{array}{l}\text { Altitude of } \\
\text { top of Aus- } \\
\text { tin Group } \\
\text { (feet above } \\
\text { NAVD 88) }\end{array}$ & $\begin{array}{l}\text { Altitude of } \\
\text { base of Aus- } \\
\text { tin Group } \\
\text { (feet above } \\
\text { NAVD 88) }\end{array}$ & $\begin{array}{c}\text { Thickness } \\
\text { of Austin } \\
\text { Group } \\
\text { (feet) }\end{array}$ & Data source & Source agency & $\begin{array}{c}\text { Data } \\
\text { source date }\end{array}$ & $\begin{array}{c}\text { Report } \\
\text { author, } \\
\text { year }^{1}\end{array}$ \\
\hline AY-68-35-616 & 855 & 661 & 537 & 124 & drillers' log & SAWS & $6 / 24 / 2002$ & unpublished data, 2002 \\
\hline AY-68-35-617 & 850 & 661 & 490 & 171 & drillers' log & SAWS & $11 / 12 / 2001$ & unpublished data, 2001 \\
\hline AY-68-35-6dd & 532 & 534 & 374 & 160 & report/publication & TWDB & -- & Livingston, 1947 \\
\hline AY-68-35-6ee & 685 & 493 & 334 & 159 & report/publication & TWDB & -- & Livingston, 1947 \\
\hline AY-68-35-6ff & 565 & 652 & 486 & 166 & geophysical log & TWDB & $11 / 5 / 1992$ & unpublished data, 1992 \\
\hline AY-68-35-6gg & 519 & 662 & 495 & 167 & geophysical log & TWDB & $5 / 13 / 1986$ & unpublished data, 1986 \\
\hline AY-68-35-705 & 1,060 & 528 & 357 & 171 & drillers' log & TWDB & $12 / 15 / 1966$ & TWDB, 2009 \\
\hline AY-68-35-710 & 735 & 520 & 345 & 175 & drillers' log & TWDB & $5 / 10 / 1985$ & TWDB, 2009 \\
\hline AY-68-35-711 & 710 & 528 & 343 & 185 & drillers' log & TWDB & $5 / 13 / 1985$ & TWDB, 2009 \\
\hline AY-68-35-712 & 735 & 545 & 360 & 185 & drillers' log & TWDB & $5 / 13 / 1985$ & TWDB, 2009 \\
\hline AY-68-35-713 & 800 & 502 & 322 & 180 & drillers' log & TWDB & $11 / 26 / 1991$ & TWDB, 2009 \\
\hline AY-68-35-7bb & -- & 498 & 324 & 174 & geophysical log & EAA & $1 / 21 / 1992$ & unpublished data, 1992 \\
\hline AY-68-35-7ee & -- & 540 & 370 & 170 & drillers' log & TWDB & $1 / 23 / 2005$ & unpublished data, 2005 \\
\hline AY-68-35-7ff & -- & 755 & 630 & 125 & drillers' log & TWDB & $2 / 18 / 2004$ & unpublished data, 2004 \\
\hline AY-68-35-7gg & 650 & 395 & 199 & 196 & geophysical log & TWDB & $3 / 16 / 1989$ & unpublished data, 1989 \\
\hline AY-68-35-7hh & 537 & 532 & 360 & 172 & geophysical log & TWDB & $4 / 26 / 1978$ & unpublished data, 1978 \\
\hline AY-68-35-801 & 837 & 426 & 274 & 152 & geophysical log & EAA & $1 / 9 / 1987$ & unpublished data, 1987 \\
\hline AY-68-35-808 & 1,150 & 450 & 304 & 146 & geophysical log & TWDB & $5 / 21 / 1973$ & TWDB, 2009 \\
\hline AY-68-35-810 & 735 & 609 & 447 & 162 & geophysical log & USGS & $5 / 8 / 1981$ & unpublished data, 1981 \\
\hline AY-68-35-811 & 735 & 702 & 432 & 270 & drillers' log & BMWD & $5 / 9 / 1981$ & unpublished data, 1981 \\
\hline AY-68-35-8bb & 1,001 & 399 & 238 & 161 & geophysical log & TWDB & 1/9/1987 & unpublished data, 1987 \\
\hline AY-68-35-903 & 1,020 & 263 & 105 & 158 & drillers' log & TWDB & 6/6/1961 & TWDB, 2009 \\
\hline AY-68-35-904 & 675 & 477 & 213 & 264 & drillers' log & SAWS & 9/8/1967 & unpublished data, 1967 \\
\hline AY-68-35-905 & 881 & 270 & 103 & 167 & drillers' log & TWDB & $1 / 1 / 1957$ & TWDB, 2009 \\
\hline AY-68-35-910 & 1,050 & 430 & 286 & 144 & drillers' log & TWDB & 9/8/1967 & Petitt and George, 1956 \\
\hline AY-68-35-912 & 1,040 & 420 & 293 & 127 & drillers' log & SAWS & $11 / 28 / 1973$ & unpublished data, 1973 \\
\hline AY-68-35-913 & 1,040 & 417 & 294 & 123 & drillers' log & SAWS & 3/5/1974 & unpublished data, 1974 \\
\hline AY $-68-36-100$ & -- & 671 & 517 & 154 & geophysical log & EAA & $12 / 2 / 1987$ & unpublished data, 1987 \\
\hline AY-68-36-102 & 786 & 854 & 704 & 150 & geophysical log & SAWS & 2/5/1963 & unpublished data, 1963 \\
\hline AY-68-36-103 & 824 & 910 & 706 & 204 & geophysical log & TWDB & 9/13/1967 & TWDB, 2009 \\
\hline AY-68-36-104 & 814 & 888 & 698 & 190 & geophysical log & TWDB & $5 / 17 / 1968$ & TWDB, 2009 \\
\hline AY-68-36-107 & 550 & 787 & 625 & 162 & drillers' log & TWDB & $12 / 28 / 1970$ & Petitt and George, 1956 \\
\hline AY-68-36-108 & 484 & 738 & 585 & 153 & drillers' log & TWDB & 9/1/1963 & TWDB, 2009 \\
\hline AY-68-36-111 & 677 & 772 & 622 & 150 & geophysical log & TWDB & $11 / 17 / 1972$ & TWDB, 2009 \\
\hline AY-68-36-121 & 356 & 795 & 654 & 141 & drillers' log & TWDB & $5 / 3 / 1905$ & TWDB, 2009 \\
\hline AY-68-36-124 & 412 & 795 & 653 & 142 & drillers' log & TWDB & $3 / 11 / 1969$ & TWDB, 2009 \\
\hline AY-68-36-129 & 450 & 790 & 625 & 165 & drillers' log & TWDB & $5 / 4 / 1972$ & TWDB, 2009 \\
\hline${ }^{2} \mathrm{AY}-68-36-134$ & 418 & 818 & 666 & 152 & drillers' log & SAWS & 7/1/1905 & USGS, 2007 \\
\hline AY-68-36-1aa & -- & 861 & 702 & 159 & geophysical log & EAA & $10 / 23 / 1986$ & unpublished data, 1986 \\
\hline AY-68-36-1bb & -- & 866 & 708 & 158 & geophysical log & EAA & $12 / 13 / 1991$ & unpublished data, 1991 \\
\hline AY-68-36-1cc & -- & 670 & 520 & 150 & geophysical log & EAA & 9/9/1989 & unpublished data, 1989 \\
\hline AY-68-36-1dd & 1,100 & 688 & 533 & 155 & report/publication & TWDB & -- & Livingston, 1947 \\
\hline AY-68-36-1ee & 498 & 708 & 554 & 154 & geophysical log & TWDB & $1 / 24 / 2000$ & TWDB, 2009 \\
\hline AY-68-36-1ff & 446 & 823 & 663 & 160 & geophysical log & TWDB & $4 / 26 / 1994$ & TWDB, 2009 \\
\hline
\end{tabular}


Appendix 1. Wells selected for inclusion in geodatabase of selected wells penetrating the Austin Group, central Bexar County, Texas,

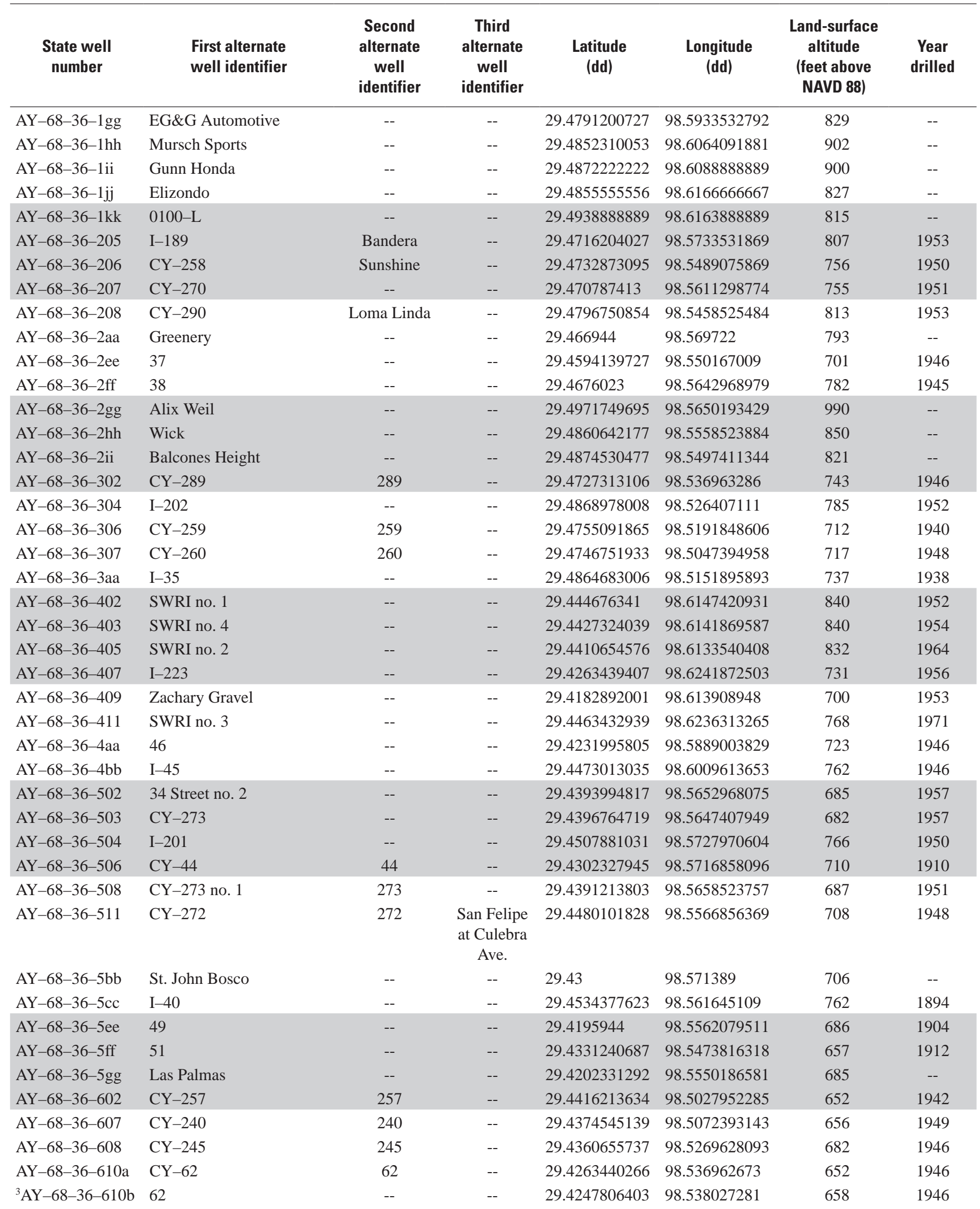


2010 - Continued.

\begin{tabular}{|c|c|c|c|c|c|c|c|c|}
\hline $\begin{array}{c}\text { State well } \\
\text { number }\end{array}$ & $\begin{array}{l}\text { Well depth } \\
\text { (feet } \\
\text { below } \\
\text { LSD) }\end{array}$ & $\begin{array}{l}\text { Altitude of } \\
\text { top of Aus- } \\
\text { tin Group } \\
\text { (feet above } \\
\text { NAVD 88) }\end{array}$ & $\begin{array}{c}\text { Altitude of } \\
\text { base of Aus- } \\
\text { tin Group } \\
\text { (feet above } \\
\text { NAVD 88) }\end{array}$ & $\begin{array}{c}\text { Thickness } \\
\text { of Austin } \\
\text { Group } \\
\text { (feet) }\end{array}$ & Data source & Source agency & $\begin{array}{c}\text { Data } \\
\text { source date }\end{array}$ & $\begin{array}{c}\text { Report } \\
\text { author, } \\
\text { year }^{1}\end{array}$ \\
\hline AY-68-36-1gg & 512 & 710 & 555 & 155 & geophysical log & TWDB & $3 / 1 / 1988$ & TWDB, 2009 \\
\hline AY-68-36-1hh & 636 & 852 & 704 & 148 & geophysical log & TWDB & $10 / 23 / 1986$ & TWDB, 2009 \\
\hline AY-68-36-1ii & 492 & 810 & 683 & 127 & geophysical log & TWDB & 7/8/1994 & TWDB, 2009 \\
\hline AY-68-36-1jj & 394 & 770 & 607 & 163 & geophysical log & TWDB & $1 / 7 / 2004$ & TWDB, 2009 \\
\hline AY-68-36-1kk & 288 & 815 & 667 & 148 & geophysical log & TWDB & $1 / 11 / 2000$ & TWDB, 2009 \\
\hline AY-68-36-205 & 956 & 641 & 543 & 98 & geophysical log & TWDB & $10 / 19 / 1953$ & Petitt and George, 1956 \\
\hline AY-68-36-206 & 748 & 524 & 376 & 148 & geophysical log & TWDB & $11 / 3 / 1950$ & Petitt and George, 1956 \\
\hline AY-68-36-207 & 1,030 & 557 & 411 & 146 & geophysical log & TWDB & $9 / 22 / 1951$ & Petitt and George, 1956 \\
\hline AY-68-36-208 & 840 & 503 & 339 & 164 & drillers' log & TWDB & $9 / 16 / 1953$ & Petitt and George, 1956 \\
\hline AY-68-36-2aа & -- & 691 & 539 & 152 & geophysical log & EAA & $8 / 23 / 1991$ & unpublished data, 1991 \\
\hline AY-68-36-2ee & 994 & 465 & 315 & 150 & report/publication & TWDB & -- & Livingston, 1947 \\
\hline AY-68-36-2ff & 524 & 572 & 434 & 138 & report/publication & TWDB & -- & Livingston, 1947 \\
\hline AY-68-36-2gg & 524 & 805 & 665 & 140 & geophysical log & TWDB & $11 / 21 / 1991$ & unpublished data, 1991 \\
\hline AY-68-36-2hh & -- & 790 & 595 & 195 & strip log & TWDB & 2/1/1946 & unpublished data, 1946 \\
\hline AY-68-36-2ii & 480 & 666 & 566 & 100 & strip log & TWDB & 4/1/1946 & unpublished data, 1946 \\
\hline AY-68-36-302 & 744 & 409 & 271 & 138 & geophysical log & TWDB & $11 / 14 / 1946$ & Petitt and George, 1956 \\
\hline AY-68-36-304 & 1,094 & 503 & 358 & 145 & geophysical log & SAWS & $3 / 16 / 1952$ & Petitt and George, 1956 \\
\hline AY-68-36-306 & 1,100 & 485 & 305 & 180 & drillers' log & SAWS & 9/1/1940 & Petitt and George, 1956 \\
\hline AY-68-36-307 & 772 & 428 & 279 & 149 & geophysical log & SAWS & $11 / 25 / 1948$ & Petitt and George, 1956 \\
\hline AY-68-36-3aа & 260 & 605 & 453 & 152 & report/publication & TWDB & -- & Livingston, 1947 \\
\hline AY-68-36-402 & 679 & 486 & 425 & 61 & drillers' log & TWDB & $5 / 12 / 1952$ & TWDB, 2009 \\
\hline AY-68-36-403 & 889 & 604 & 448 & 156 & geophysical log & TWDB & $2 / 25 / 1986$ & TWDB, 2009 \\
\hline AY-68-36-405 & 780 & 581 & 423 & 158 & drillers' log & TWDB & 4/6/1964 & TWDB, 2009 \\
\hline AY-68-36-407 & 620 & 508 & 356 & 152 & geophysical log & USGS & $11 / 29 / 1971$ & unpublished data, 1971 \\
\hline AY-68-36-409 & 1,215 & 289 & 140 & 149 & drillers' log & TWDB & $10 / 9 / 1953$ & TWDB, 2009 \\
\hline AY-68-36-411 & 800 & 574 & 392 & 182 & drillers' log & TWDB & $12 / 20 / 1974$ & TWDB, 2009 \\
\hline AY-68-36-4aa & 965 & 23 & -137 & 160 & report/publication & TWDB & -- & Livingston, 1947 \\
\hline AY-68-36-4bb & 490 & 602 & 452 & 150 & report/publication & TWDB & -- & Livingston, 1947 \\
\hline AY-68-36-502 & 1,217 & 261 & 116 & 145 & drillers' log & SAWS & $10 / 7 / 1957$ & unpublished data, 1957 \\
\hline AY-68-36-503 & 1,240 & 251 & 119 & 132 & geophysical log & SAWS & $10 / 28 / 1951$ & unpublished data, 1951 \\
\hline AY-68-36-504 & 772 & 454 & 306 & 148 & geophysical log & TWDB & $7 / 10 / 1950$ & Petitt and George, 1956 \\
\hline AY-68-36-506 & 1,000 & 320 & 182 & 138 & report/publication & TWDB & -- & Livingston, 1947 \\
\hline AY-68-36-508 & 950 & 257 & 127 & 130 & drillers' log & TWDB & $11 / 26 / 1951$ & Petitt and George, 1956 \\
\hline AY-68-36-511 & 1,051 & 156 & 38 & 118 & drillers' log & SAWS & 8/1/1948 & Petitt and George, 1956 \\
\hline AY-68-36-5bb & -- & 464 & 174 & 290 & geophysical log & EAA & $5 / 2 / 1995$ & unpublished data, 1995 \\
\hline AY-68-36-5cc & 702 & 474 & 307 & 167 & report/publication & TWDB & -- & Livingston, 1947 \\
\hline AY-68-36-5ee & 1,286 & -125 & -265 & 140 & report/publication & TWDB & -- & Livingston, 1947 \\
\hline AY-68-36-5ff & 609 & 470 & 308 & 162 & report/publication & TWDB & -- & Livingston, 1947 \\
\hline AY-68-36-5gg & 1,253 & -25 & -225 & 200 & geophysical log & TWDB & 6/3/1992 & unpublished data, 1992 \\
\hline AY-68-36-602 & 886 & 291 & 112 & 179 & drillers' log & SAWS & 2/1/1942 & Petitt and George, 1956 \\
\hline AY-68-36-607 & 1,221 & 223 & 75 & 148 & drillers' log & TWDB & 2/1/1949 & Petitt and George, 1956 \\
\hline AY-68-36-608 & 554 & 476 & 387 & 89 & drillers' log & TWDB & $10 / 1 / 1946$ & Petitt and George, 1956 \\
\hline AY-68-36-610a & 1,212 & -13 & -168 & 155 & geophysical log & TWDB & 4/11/1946 & Petitt and George, 1956 \\
\hline${ }^{3} \mathrm{AY}-68-36-610 b$ & 1,212 & 189 & 1 & 188 & report/publication & TWDB & -- & Livingston, 1947 \\
\hline
\end{tabular}


Appendix 1. Wells selected for inclusion in geodatabase of selected wells penetrating the Austin Group, central Bexar County, Texas,

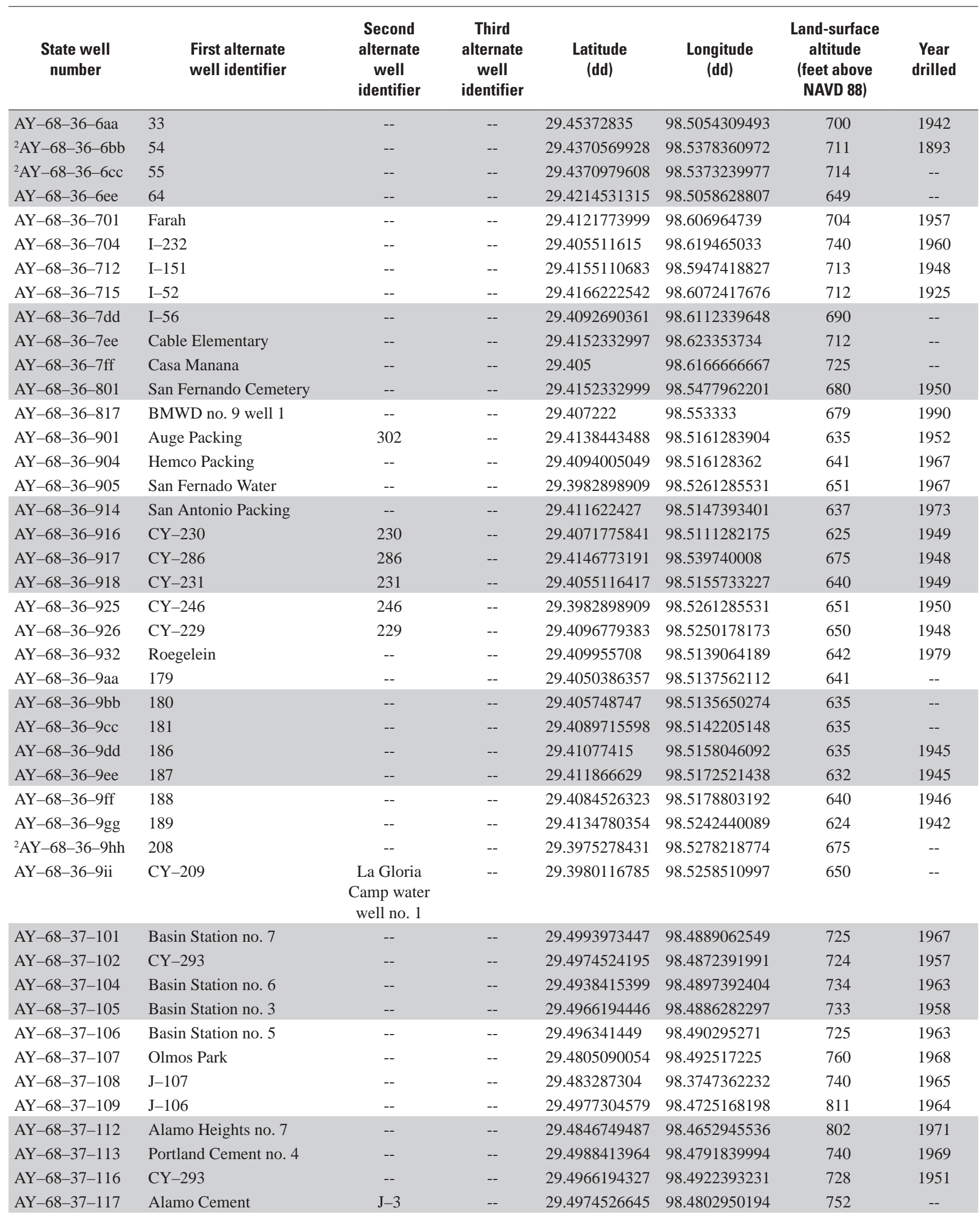




\begin{tabular}{|c|c|c|c|c|c|c|c|c|}
\hline $\begin{array}{c}\text { State well } \\
\text { number }\end{array}$ & $\begin{array}{l}\text { Well depth } \\
\text { (feet } \\
\text { below } \\
\text { LSD) }\end{array}$ & $\begin{array}{l}\text { Altitude of } \\
\text { top of Aus- } \\
\text { tin Group } \\
\text { (feet above } \\
\text { NAVD 88) }\end{array}$ & $\begin{array}{l}\text { Altitude of } \\
\text { base of Aus- } \\
\text { tin Group } \\
\text { (feet above } \\
\text { NAVD 88) }\end{array}$ & $\begin{array}{c}\text { Thickness } \\
\text { of Austin } \\
\text { Group } \\
\text { (feet) }\end{array}$ & Data source & Source agency & $\begin{array}{c}\text { Data } \\
\text { source date }\end{array}$ & $\begin{array}{c}\text { Report } \\
\text { author, } \\
\text { year }^{1}\end{array}$ \\
\hline AY-68-36-6aа & 851 & 562 & 416 & 146 & report/publication & TWDB & -- & Livingston, 1947 \\
\hline${ }^{2} \mathrm{AY}-68-36-6 \mathrm{bb}$ & 300 & -- & -- & -- & report/publication & TWDB & -- & Livingston, 1947 \\
\hline${ }^{2} \mathrm{AY}-68-36-6 \mathrm{cc}$ & -- & -- & -- & -- & report/publication & TWDB & -- & Livingston, 1947 \\
\hline AY-68-36-6ee & 911 & 264 & 83 & 181 & report/publication & TWDB & -- & Livingston, 1947 \\
\hline AY-68-36-701 & 1,199 & 94 & -76 & 170 & drillers' log & TWDB & $2 / 26 / 1957$ & TWDB, 2009 \\
\hline AY-68-36-704 & 1,500 & -60 & -251 & 191 & geophysical log & TWDB & $2 / 4 / 1960$ & TWDB, 2009 \\
\hline AY-68-36-712 & 1,619 & -144 & -258 & 114 & drillers' log & TWDB & $7 / 1 / 1947$ & Petitt and George, 1956 \\
\hline AY-68-36-715 & 1,006 & 106 & -95 & 201 & report/publication & TWDB & 4/8/1905 & Livingston, 1947 \\
\hline AY-68-36-7dd & 1,403 & -410 & -610 & 200 & report/publication & TWDB & -- & Livingston, 1947 \\
\hline AY-68-36-7ee & 676 & 410 & 250 & 160 & geophysical log & TWDB & $12 / 16 / 1985$ & unpublished data, 1985 \\
\hline AY-68-36-7ff & 974 & 165 & -23 & 188 & geophysical log & TWDB & 11/7/1997 & unpublished data, 1997 \\
\hline AY-68-36-801 & 1,270 & 70 & -69 & 139 & drillers' log & TWDB & $11 / 8 / 1950$ & TWDB, 2009 \\
\hline AY-68-36-817 & 1,183 & 84 & -49 & 133 & drillers' log & BMWD & $3 / 20 / 1990$ & unpublished data, 1990 \\
\hline AY-68-36-901 & 875 & 80 & -35 & 115 & drillers' log & TWDB & $1 / 10 / 1952$ & TWDB, 2009 \\
\hline AY-68-36-904 & 1,356 & 185 & -64 & 249 & drillers' log & TWDB & $3 / 13 / 1967$ & TWDB, 2009 \\
\hline AY-68-36-905 & 1,065 & 28 & -141 & 169 & drillers' log & TWDB & $10 / 14 / 1967$ & Petitt and George, 1956 \\
\hline AY-68-36-914 & 1,214 & 130 & -40 & 170 & drillers' log & TWDB & $5 / 1 / 1973$ & TWDB, 2009 \\
\hline AY-68-36-916 & 1,289 & 164 & 15 & 149 & geophysical log & TWDB & $3 / 19 / 1949$ & Petitt and George, 1956 \\
\hline AY-68-36-917 & 1,054 & 95 & -61 & 156 & geophysical log & SAWS & $2 / 26 / 1948$ & Petitt and George, 1956 \\
\hline AY-68-36-918 & 1,351 & 133 & -15 & 148 & drillers' log & TWDB & 4/1/1949 & Petitt and George, 1956 \\
\hline AY-68-36-925 & 1,006 & -107 & -139 & 32 & drillers' log & TWDB & $3 / 6 / 1950$ & Petitt and George, 1956 \\
\hline AY-68-36-926 & 915 & 44 & -100 & 144 & drillers' log & TWDB & $5 / 1 / 1905$ & Petitt and George, 1956 \\
\hline AY-68-36-932 & 1,265 & 82 & -73 & 155 & drillers' log & TWDB & $9 / 16 / 1979$ & TWDB, 2009 \\
\hline AY-68-36-9aa & 1,400 & 341 & 25 & 316 & report/publication & TWDB & -- & Livingston, 1947 \\
\hline AY-68-36-9bb & 1,250 & 295 & -21 & 316 & report/publication & TWDB & -- & Livingston, 1947 \\
\hline AY-68-36-9cc & 1,400 & 295 & -21 & 316 & report/publication & TWDB & -- & Livingston, 1947 \\
\hline AY-68-36-9dd & 948 & 262 & -9 & 271 & report/publication & TWDB & -- & Livingston, 1947 \\
\hline AY-68-36-9ee & 851 & 172 & -41 & 213 & report/publication & TWDB & -- & Livingston, 1947 \\
\hline AY-68-36-9ff & 1,126 & 84 & -65 & 149 & report/publication & TWDB & -- & Livingston, 1947 \\
\hline AY-68-36-9gg & 1,011 & 283 & 23 & 260 & report/publication & TWDB & -- & Livingston, 1947 \\
\hline${ }^{2} \mathrm{AY}-68-36-9 \mathrm{hh}$ & 700 & -- & -- & -- & report/publication & TWDB & -- & Livingston, 1947 \\
\hline AY-68-36-9ii & -- & -115 & -175 & 60 & geophysical log & TWDB & 9/6/1948 & Petitt and George, 1956 \\
\hline AY-68-37-101 & 995 & 438 & 299 & 139 & drillers' log & SAWS & $3 / 21 / 1967$ & unpublished data, 1967 \\
\hline AY-68-37-102 & 1,002 & 482 & 345 & 137 & geophysical log & SAWS & $7 / 26 / 1951$ & unpublished data, 1951 \\
\hline AY-68-37-104 & 995 & 444 & 306 & 138 & geophysical log & SAWS & $1 / 31 / 1963$ & unpublished data, 1963 \\
\hline AY-68-37-105 & 1,044 & 447 & 311 & 136 & drillers' log & SAWS & $3 / 12 / 1958$ & unpublished data, 1958 \\
\hline AY-68-37-106 & 986 & 460 & 325 & 135 & geophysical log & SAWS & $12 / 4 / 1962$ & unpublished data, 1962 \\
\hline AY-68-37-107 & 816 & 392 & 292 & 100 & geophysical log & USGS & $9 / 16 / 1971$ & unpublished data, 1971 \\
\hline AY-68-37-108 & 986 & 398 & 260 & 138 & drillers' log & TWDB & $6 / 3 / 1965$ & TWDB, 2009 \\
\hline AY-68-37-109 & 450 & 811 & 670 & 141 & drillers' log & TWDB & 9/1/1964 & TWDB, 2009 \\
\hline AY-68-37-112 & 592 & 654 & 540 & 114 & geophysical log & TWDB & $5 / 19 / 1971$ & Petitt and George, 1956 \\
\hline AY-68-37-113 & 805 & 396 & 258 & 138 & drillers' log & TWDB & $11 / 26 / 1969$ & TWDB, 2009 \\
\hline AY-68-37-116 & 700 & 483 & 358 & 125 & drillers' log & TWDB & $8 / 22 / 1951$ & Petitt and George, 1956 \\
\hline AY-68-37-117 & 705 & 416 & 292 & 124 & geophysical log & TWDB & $7 / 2 / 1986$ & Petitt and George, 1956 \\
\hline
\end{tabular}


Appendix 1. Wells selected for inclusion in geodatabase of selected wells penetrating the Austin Group, central Bexar County, Texas,

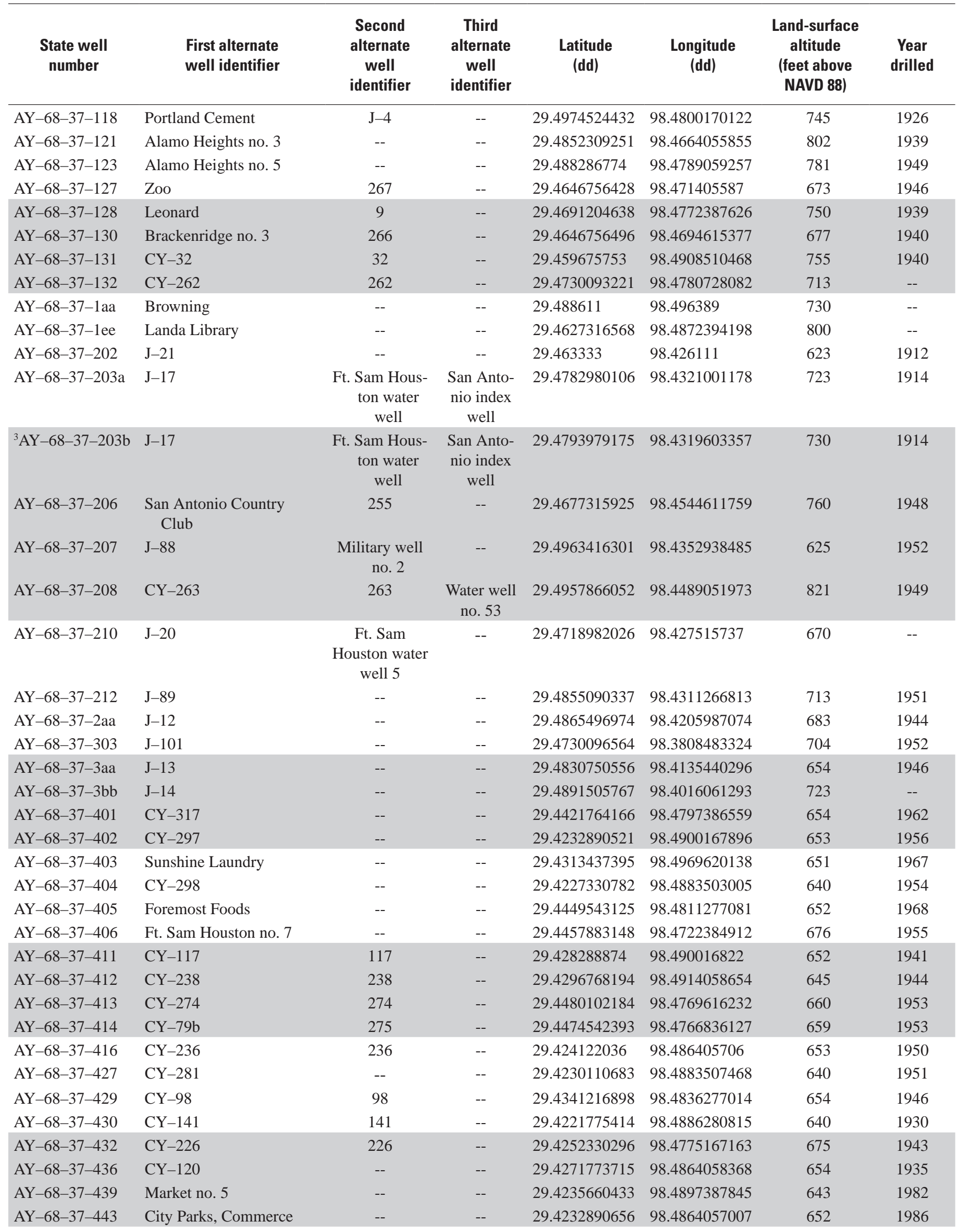


2010-Continued.

\begin{tabular}{|c|c|c|c|c|c|c|c|c|}
\hline $\begin{array}{c}\text { State well } \\
\text { number }\end{array}$ & $\begin{array}{l}\text { Well depth } \\
\text { (feet } \\
\text { below } \\
\text { LSD) }\end{array}$ & $\begin{array}{c}\text { Altitude of } \\
\text { top of Aus- } \\
\text { tin Group } \\
\text { (feet above } \\
\text { NAVD 88) }\end{array}$ & $\begin{array}{l}\text { Altitude of } \\
\text { base of Aus- } \\
\text { tin Group } \\
\text { (feet above } \\
\text { NAVD 88) }\end{array}$ & $\begin{array}{c}\text { Thickness } \\
\text { of Austin } \\
\text { Group } \\
\text { (feet) }\end{array}$ & Data source & Source agency & $\begin{array}{c}\text { Data } \\
\text { source date }\end{array}$ & $\begin{array}{c}\text { Report } \\
\text { author, } \\
\text { year }\end{array}$ \\
\hline AY-68-37-118 & 700 & 411 & 285 & 126 & drillers' log & TWDB & 4/9/1905 & Petitt and George, 1956 \\
\hline AY-68-37-121 & 603 & 657 & 577 & 80 & drillers' log & TWDB & 8/2/1939 & Petitt and George, 1956 \\
\hline AY-68-37-123 & 580 & 781 & 623 & 158 & drillers' log & TWDB & $9 / 16 / 1949$ & TWDB, 2009 \\
\hline AY-68-37-127 & 407 & 673 & 575 & 98 & drillers' log & TWDB & 9/20/1946 & Petitt and George, 1956 \\
\hline AY-68-37-128 & 209 & 750 & 617 & 133 & drillers' log & TWDB & $4 / 22 / 1905$ & Petitt and George, 1956 \\
\hline AY-68-37-130 & 800 & 677 & 582 & 95 & drillers' log & TWDB & $4 / 23 / 1905$ & Petitt and George, 1956 \\
\hline AY-68-37-131 & 650 & 755 & 637 & 118 & drillers' log & TWDB & $10 / 1 / 1940$ & Petitt and George, 1956 \\
\hline AY-68-37-132 & -- & 713 & 693 & 20 & geophysical log & SAWS & $11 / 6 / 1951$ & Petitt and George, 1956 \\
\hline AY-68-37-1 aa & -- & 492 & 355 & 137 & geophysical log & SAWS & $11 / 5 / 1997$ & unpublished data, 1997 \\
\hline AY-68-37-1ee & 370 & 800 & 680 & 120 & geophysical log & TWDB & $3 / 17 / 1966$ & unpublished data, 1966 \\
\hline AY-68-37-202 & 702 & 298 & 180 & 118 & drillers' log & EAA & 6/1/1984 & Petitt and George, 1956 \\
\hline AY-68-37-203a & 874 & 533 & 370 & 163 & report/publication & TWDB & -- & Livingston, 1947 \\
\hline${ }^{3} \mathrm{AY}-68-37-203 b$ & 874 & 540 & 377 & 163 & geophysical log & TWDB & $3 / 5 / 1971$ & Petitt and George, 1956 \\
\hline AY-68-37-206 & 921 & 253 & 118 & 135 & drillers' log & TWDB & $12 / 29 / 1948$ & Petitt and George, 1956 \\
\hline AY-68-37-207 & 872 & 185 & 95 & 90 & drillers' log & SAWS & 2/13/1952 & Petitt and George, 1956 \\
\hline AY-68-37-208 & 763 & 706 & 538 & 168 & drillers' log & TWDB & $8 / 26 / 1949$ & Petitt and George, 1956 \\
\hline AY-68-37-210 & 660 & 376 & 240 & 136 & geophysical log & TWDB & $10 / 14 / 1969$ & Petitt and George, 1956 \\
\hline AY-68-37-212 & 778 & 528 & 393 & 135 & geophysical log & SAWS & $4 / 15 / 1951$ & Petitt and George, 1956 \\
\hline AY-68-37-2aa & 460 & 493 & 365 & 128 & report/publication & TWDB & -- & Livingston, 1947 \\
\hline AY-68-37-303 & 685 & 384 & 245 & 139 & drillers' log & TWDB & $3 / 8 / 1961$ & TWDB, 2009 \\
\hline AY-68-37-3aа & 735 & 464 & 336 & 128 & report/publication & TWDB & -- & Livingston, 1947 \\
\hline AY-68-37-3bb & 802 & 519 & 370 & 149 & report/publication & TWDB & -- & Livingston, 1947 \\
\hline AY-68-37-401 & 1,106 & 272 & 154 & 118 & drillers' log & TWDB & $11 / 13 / 1962$ & TWDB, 2009 \\
\hline AY-68-37-402 & 1,162 & 306 & 153 & 153 & geophysical log & TWDB & $10 / 11 / 1956$ & TWDB, 2009 \\
\hline AY-68-37-403 & 1,089 & 285 & 131 & 154 & drillers' log & TWDB & 9/14/1967 & TWDB, 2009 \\
\hline AY-68-37-404 & 1,326 & 168 & -18 & 186 & geophysical log & TWDB & $1 / 25 / 1954$ & TWDB, 2009 \\
\hline AY-68-37-405 & 775 & 468 & 166 & 302 & geophysical log & EAA & $3 / 21 / 1990$ & unpublished data, 1990 \\
\hline AY-68-37-406 & 1,103 & 290 & 142 & 148 & geophysical log & TWDB & $8 / 4 / 1955$ & TWDB, 2009 \\
\hline AY-68-37-411 & 805 & 251 & 97 & 154 & report/publication & TWDB & $6 / 14 / 1946$ & Livingston, 1947 \\
\hline AY-68-37-412 & 1,157 & 240 & 89 & 151 & drillers' log & TWDB & $3 / 14 / 1951$ & Petitt and George, 1956 \\
\hline AY-68-37-413 & 1,000 & 294 & 140 & 154 & geophysical log & SAWS & $1 / 29 / 1953$ & Petitt and George, 1956 \\
\hline AY-68-37-414 & 1,160 & 472 & 134 & 338 & drillers' log & SAWS & $4 / 26 / 1954$ & Petitt and George, 1956 \\
\hline AY-68-37-416 & 1,113 & 320 & 15 & 305 & drillers' log & TWDB & 3/1/1951 & Petitt and George, 1956 \\
\hline AY-68-37-427 & 1,140 & 160 & 7 & 153 & geophysical log & SAWS & $6 / 16 / 1951$ & unpublished data, 1951 \\
\hline AY-68-37-429 & 749 & 291 & 143 & 148 & geophysical log & TWDB & $7 / 22 / 1946$ & Petitt and George, 1956 \\
\hline AY-68-37-430 & 900 & 330 & 155 & 175 & drillers' log & TWDB & $1 / 1 / 1931$ & Petitt and George, 1956 \\
\hline AY-68-37-432 & 966 & 285 & 130 & 155 & drillers' log & TWDB & $2 / 20 / 1951$ & Petitt and George, 1956 \\
\hline AY-68-37-436 & 1,159 & 262 & 105 & 157 & drillers' log & TWDB & $7 / 24 / 1946$ & TWDB, 2009 \\
\hline AY-68-37-439 & 1,280 & 157 & 27 & 130 & drillers' log & SAWS & $4 / 19 / 1982$ & TWDB, 2009 \\
\hline AY $-68-37-443$ & 1,252 & 172 & 25 & 147 & drillers' log & TWDB & 7/1/1986 & TWDB, 2009 \\
\hline
\end{tabular}


Appendix 1. Wells selected for inclusion in geodatabase of selected wells penetrating the Austin Group, central Bexar County, Texas,

\begin{tabular}{|c|c|c|c|c|c|c|c|}
\hline $\begin{array}{l}\text { State well } \\
\text { number }\end{array}$ & $\begin{array}{l}\text { First alternate } \\
\text { well identifier }\end{array}$ & $\begin{array}{c}\text { Second } \\
\text { alternate } \\
\text { well } \\
\text { identifier }\end{array}$ & $\begin{array}{l}\text { Third } \\
\text { alternate } \\
\text { well } \\
\text { identifier }\end{array}$ & $\begin{array}{l}\text { Latitude } \\
\text { (dd) }\end{array}$ & $\begin{array}{l}\text { Longitude } \\
\text { (dd) }\end{array}$ & $\begin{array}{l}\text { Land-surface } \\
\text { altitude } \\
\text { (feet above } \\
\text { NAVD 88) }\end{array}$ & $\begin{array}{l}\text { Year } \\
\text { drilled }\end{array}$ \\
\hline AY-68-37-4aa & 80 & -- & -- & 29.4456151237 & 98.473440534 & 673 & 1903 \\
\hline AY-68-37-4bb & 82 & -- & -- & 29.4448777005 & 98.4830543485 & 656 & 1931 \\
\hline AY-68-37-4ff & 123 & -- & -- & 29.4253587437 & 98.4894212432 & 631 & 1905 \\
\hline AY-68-37-4gg & 126 & -- & -- & 29.4236107774 & 98.487564029 & 647 & 1905 \\
\hline AY-68-37-4hh & 144 & -- & -- & 29.4238292732 & 98.4929717997 & 647 & 1929 \\
\hline AY-68-37-4ii & 140 & -- & -- & 29.4221632428 & 98.4894758671 & 642 & 1928 \\
\hline AY-68-37-4jj & CWB no. 5 & -- & -- & 29.4230108408 & 98.4897392254 & 650 & -- \\
\hline AY-68-37-509 & Coca Cola no. 2 & -- & -- & 29.4224552737 & 98.4389045267 & 631 & 1966 \\
\hline AY-68-37-510 & Coca Cola & -- & -- & 29.4221772785 & 98.4402935593 & 630 & 1974 \\
\hline AY-68-37-511 & $\mathrm{J}-24$ & -- & -- & 29.439166 & 98.429443 & 642 & 1940 \\
\hline AY-68-37-517 & SFD Enterprises & -- & -- & 29.4421770618 & 98.424459944 & 635 & -- \\
\hline AY-68-37-518 & CY-277 & 277 & -- & 29.4330106896 & 98.4341823579 & 639 & -- \\
\hline AY-68-37-519 & Artesia no. 6 & -- & -- & 29.4341218544 & 98.4389045923 & 649 & 1975 \\
\hline AY-68-37-520 & CY-276 & 276 & -- & 29.4330109046 & 98.4361265172 & 637 & 1949 \\
\hline AY-68-37-521 & Defose no. 1 & $\mathrm{~A}-1$ & -- & 29.4182894644 & 98.4280712375 & 620 & 1985 \\
\hline AY $-68-37-523$ & Defose no. $3 \mathrm{a}$ & A-3 & -- & 29.4182894655 & 98.4277932307 & 620 & 1985 \\
\hline AY-68-37-524 & Willow Springs no. 1C & $\mathrm{C}-1$ & -- & 29.4288440617 & 98.4341824459 & 626 & 1986 \\
\hline AY-68-37-601 & Suburban no. 2 & $\mathrm{~J}-87$ & -- & 29.4455103433 & 98.4055706008 & 686 & 1940 \\
\hline AY-68-37-605 & Kirby & -- & -- & 29.4521768056 & 98.3952925987 & 684 & 1971 \\
\hline AY-68-37-607 & $\mathrm{J}-69$ & -- & -- & 29.4449548068 & 98.4058483827 & 684 & 1947 \\
\hline$A Y-68-37-608$ & $\mathrm{~J}-79$ & -- & -- & 29.4566212475 & 98.3819592845 & 688 & 1944 \\
\hline AY-68-37-610 & Seale Road no. 4 & -- & -- & 29.445278 & 98.405833 & 695 & 1996 \\
\hline AY-68-37-714 & L \& H Packing & 241 & -- & 29.3944010833 & 98.4894615887 & 612 & 1947 \\
\hline AY-68-37-716 & 153 & -- & -- & 29.4146773652 & 98.4883506929 & 630 & 1939 \\
\hline AY-68-37-719 & CY-294 & 294 & -- & 29.4163443332 & 98.4811275267 & 649 & 1952 \\
\hline AY-68-37-720 & L \& H Packing no. 2 & -- & -- & 29.3949560614 & 98.4900166057 & 610 & 1985 \\
\hline AY-68-37-721 & L \& H Packing no. 3 & -- & -- & 29.395 & 98.490278 & 621 & 1997 \\
\hline AY-68-37-7aa & 150 & -- & -- & 29.4120851248 & 98.496740852 & 630 & 1912 \\
\hline$A Y-68-37-7 b b$ & 151 & -- & -- & 29.4109926458 & 98.4978606429 & 632 & 1929 \\
\hline AY-68-37-7cc & Blue Star & -- & -- & 29.4146778096 & 98.4883502485 & 636 & -- \\
\hline AY-68-37-7dd & 163 & -- & -- & 29.3976917149 & 98.4901313545 & 598 & -- \\
\hline AY-68-37-7ee & 164 & SAWS no. 4 & -- & 29.3976917149 & 98.4901313545 & 597 & 1941 \\
\hline AY-68-38-104 & BMWD-Northeast & 036 WP no. 1 & -- & 29.4910640241 & 98.373903237 & 744 & 1968 \\
\hline
\end{tabular}


2010-Continued.

\begin{tabular}{|c|c|c|c|c|c|c|c|c|}
\hline $\begin{array}{l}\text { State well } \\
\text { number }\end{array}$ & $\begin{array}{c}\text { Well depth } \\
\text { (feet } \\
\text { below } \\
\text { LSD) }\end{array}$ & $\begin{array}{c}\text { Altitude of } \\
\text { top of Aus- } \\
\text { tin Group } \\
\text { (feet above } \\
\text { NAVD 88) }\end{array}$ & $\begin{array}{l}\text { Altitude of } \\
\text { base of Aus- } \\
\text { tin Group } \\
\text { (feet above } \\
\text { NAVD 88) }\end{array}$ & $\begin{array}{c}\text { Thickness } \\
\text { of Austin } \\
\text { Group } \\
\text { (feet) }\end{array}$ & Data source & Source agency & $\begin{array}{c}\text { Data } \\
\text { source date }\end{array}$ & $\begin{array}{c}\text { Report } \\
\text { author, } \\
\text { year }^{1}\end{array}$ \\
\hline AY-68-37-4aa & 729 & 319 & 138 & 181 & report/publication & TWDB & -- & Livingston, 1947 \\
\hline AY-68-37-4bb & 853 & 301 & 151 & 150 & report/publication & TWDB & -- & Livingston, 1947 \\
\hline AY-68-37-4dd & 1,159 & 259 & 102 & 157 & report/publication & TWDB & -- & Livingston, 1947 \\
\hline AY-68-37-4ee & 1,043 & 213 & 65 & 148 & report/publication & TWDB & -- & Livingston, 1947 \\
\hline AY-68-37-4ff & 747 & 211 & 76 & 135 & report/publication & TWDB & -- & Livingston, 1947 \\
\hline AY-68-37-4gg & 961 & 163 & 19 & 144 & report/publication & TWDB & -- & Livingston, 1947 \\
\hline AY-68-37-4hh & 968 & 179 & 62 & 117 & report/publication & TWDB & -- & Livingston, 1947 \\
\hline AY-68-37-4ii & 912 & 130 & 9 & 121 & report/publication & TWDB & -- & Livingston, 1947 \\
\hline AY-68-37-4jj & 777 & 176 & 34 & 142 & geophysical log & TWDB & $10 / 1 / 1981$ & unpublished data, 1981 \\
\hline AY-68-37-4kk & 713 & 296 & 154 & 142 & geophysical log & TWDB & $4 / 12 / 1988$ & unpublished data, 1988 \\
\hline AY-68-37-507 & 1,108 & 42 & -73 & 115 & drillers' log & TWDB & $4 / 4 / 1954$ & Petitt and George, 1956 \\
\hline AY-68-37-508 & 1,318 & -158 & -249 & 91 & drillers' log & SAWS & $1 / 3 / 1958$ & unpublished data, 1958 \\
\hline AY-68-37-509 & 901 & 45 & -120 & 165 & drillers' log & TWDB & $3 / 8 / 1974$ & TWDB, 2009 \\
\hline AY-68-37-510 & 1,150 & -54 & -74 & 20 & drillers' log & TWDB & $4 / 21 / 1966$ & TWDB, 2009 \\
\hline AY-68-37-511 & 904 & 92 & -106 & 198 & report/publication & TWDB & -- & Livingston, 1947 \\
\hline AY-68-37-517 & 928 & 50 & -75 & 125 & drillers' log & TWDB & $1 / 11 / 1952$ & TWDB, 2009 \\
\hline AY-68-37-518 & 945 & 134 & -61 & 195 & geophysical log & TWDB & $11 / 26 / 1951$ & Petitt and George, 1956 \\
\hline AY-68-37-519 & 1,340 & 0 & -116 & 116 & drillers' log & SAWS & $10 / 14 / 1975$ & unpublished data, 1975 \\
\hline AY $-68-37-520$ & 1,000 & 21 & -97 & 118 & drillers' log & TWDB & 1/1/1949 & Petitt and George, 1956 \\
\hline AY-68-37-521 & 1,489 & -80 & -192 & 112 & geophysical log & TWDB & $8 / 1 / 1985$ & TWDB, 2009 \\
\hline AY-68-37-523 & 1,175 & -80 & -193 & 113 & geophysical log & TWDB & $10 / 15 / 1985$ & TWDB, 2009 \\
\hline AY-68-37-524 & 891 & 74 & -44 & 118 & geophysical log & SAWS & $11 / 25 / 1985$ & unpublished data, 1985 \\
\hline AY $-68-37-525$ & 1,150 & 76 & -46 & 122 & geophysical log & EAA & $1 / 17 / 1985$ & unpublished data, 1985 \\
\hline AY $-68-37-526$ & 1,223 & 62 & -68 & 130 & geophysical log & EAA & $2 / 21 / 1986$ & unpublished data, 1986 \\
\hline AY-68-37-527 & 926 & 47 & -77 & 124 & drillers' log & SAWS & $5 / 6 / 1986$ & unpublished data, 1986 \\
\hline AY-68-37-528 & 885 & 249 & & 250 & drillers' log & TWDB & $7 / 23 / 1985$ & TWDB, 2009 \\
\hline AY-68-37-5bb & 867 & 47 & -77 & 124 & geophysical log & TWDB & $5 / 7 / 1986$ & unpublished data, 1986 \\
\hline AY-68-37-5cc & 994 & 99 & -96 & 195 & geophysical log & TWDB & $10 / 4 / 1991$ & unpublished data, 1991 \\
\hline AY-68-37-601 & 1,150 & -24 & -164 & 140 & geophysical log & TWDB & $3 / 9 / 1953$ & Petitt and George, 1956 \\
\hline AY-68-37-605 & 1,139 & -40 & -186 & 146 & drillers' log & TWDB & $5 / 7 / 1971$ & TWDB, 2009 \\
\hline AY-68-37-607 & 1,054 & -43 & -163 & 120 & drillers' log & TWDB & $7 / 1 / 1947$ & Petitt and George, 1956 \\
\hline AY-68-37-608 & 1,047 & 18 & -180 & 198 & geophysical log & TWDB & $10 / 1 / 1944$ & Petitt and George, 1956 \\
\hline AY-68-37-610 & 1,145 & -35 & -145 & 110 & drillers' log & SAWS & $5 / 17 / 1996$ & unpublished data, 1996 \\
\hline AY-68-37-714 & 1,260 & -307 & -423 & 116 & drillers' log & TWDB & $8 / 1 / 1947$ & Petitt and George, 1956 \\
\hline AY-68-37-716 & 844 & 247 & 22 & 225 & report/publication & TWDB & -- & Livingston, 1947 \\
\hline AY-68-37-719 & 1,241 & 148 & 5 & 143 & geophysical log & SAWS & $4 / 17 / 1952$ & Petitt and George, 1956 \\
\hline AY-68-37-720 & 1,286 & -350 & -440 & 90 & drillers' log & TWDB & $3 / 17 / 1988$ & TWDB, 2009 \\
\hline AY-68-37-721 & 1,475 & 0 & -176 & 176 & drillers' log & TWDB & 1/3/1997 & TWDB, 2009 \\
\hline AY-68-37-7aa & 758 & 188 & 50 & 138 & report/publication & TWDB & -- & Livingston, 1947 \\
\hline AY-68-37-7bb & 1,652 & 162 & 57 & 105 & report/publication & TWDB & -- & Livingston, 1947 \\
\hline AY-68-37-7cc & -- & 116 & -22 & 138 & geophysical log & EAA & 9/28/1992 & unpublished data, 1992 \\
\hline AY-68-37-7dd & 998 & -302 & -599 & 297 & report/publication & TWDB & -- & Livingston, 1947 \\
\hline AY-68-37-7ee & 1,052 & 69 & -184 & 253 & report/publication & TWDB & -- & Livingston, 1947 \\
\hline AY-68-38-104 & 998 & 579 & 331 & 248 & drillers' log & TWDB & $7 / 2 / 1968$ & TWDB, 2009 \\
\hline
\end{tabular}


Appendix 1. Wells selected for inclusion in geodatabase of selected wells penetrating the Austin Group, central Bexar County, Texas,

\begin{tabular}{cllccccc}
\hline $\begin{array}{c}\text { State well } \\
\text { number }\end{array}$ & \multicolumn{1}{c}{$\begin{array}{c}\text { First alternate } \\
\text { well identifier }\end{array}$} & $\begin{array}{c}\text { Second } \\
\text { alternate } \\
\text { well } \\
\text { identifier }\end{array}$ & $\begin{array}{c}\text { Third } \\
\text { alternate } \\
\text { well } \\
\text { identifier }\end{array}$ & $\begin{array}{c}\text { Latitude } \\
\text { (dd) }\end{array}$ & $\begin{array}{c}\text { Longitude } \\
\text { (dd) }\end{array}$ & $\begin{array}{c}\text { Land-surface } \\
\text { altitude } \\
\text { (feet above } \\
\text { NAVD 88) }\end{array}$ & $\begin{array}{c}\text { Year } \\
\text { drilled }\end{array}$ \\
\hline AY-68-38-106 & Doug Sanders & -- & -- & 29.4832873536 & 98.3600138458 & 745 & 1970 \\
AY-68-38-108 & Ray Ellison & -- & -- & 29.483287304 & 98.3747362232 & 730 \\
AY-68-38-109 & J-41 & -- & -- & 29.4666210053 & 98.3472354505 & 687 & 1971 \\
AY-68-38-110 & Doug Sanders & -- & -- & 29.4821753632 & 98.3691810759 & 715 & 1973 \\
AY-68-38-111 & Lackland & -- & -- & 29.4971749217 & 98.3375133228 & 800 & 1985 \\
AY-68-38-1aa & W.M. Collier & -- & -- & 29.4622222222 & 98.3738888889 & 697 & -- \\
\hline
\end{tabular}

${ }^{1}$ Unpublished data on file at source agency.

${ }^{2}$ Completed only in Austin Group.

${ }^{3}$ Duplicate of proceeding well entry with different well information for some fields; determination of which well record contained the correct well information was not possible for this report. 
2010-Continued.

\begin{tabular}{|c|c|c|c|c|c|c|c|c|}
\hline $\begin{array}{c}\text { State well } \\
\text { number }\end{array}$ & $\begin{array}{l}\text { Well depth } \\
\text { (feet } \\
\text { below } \\
\text { LSD) }\end{array}$ & $\begin{array}{c}\text { Altitude of } \\
\text { top of Aus- } \\
\text { tin Group } \\
\text { (feet above } \\
\text { NAVD 88) }\end{array}$ & $\begin{array}{c}\text { Altitude of } \\
\text { base of Aus- } \\
\text { tin Group } \\
\text { (feet above } \\
\text { NAVD 88) }\end{array}$ & $\begin{array}{c}\text { Thickness } \\
\text { of Austin } \\
\text { Group } \\
\text { (feet) }\end{array}$ & Data source & Source agency & $\begin{array}{c}\text { Data } \\
\text { source date }\end{array}$ & $\begin{array}{c}\text { Report } \\
\text { author, } \\
\text { year }\end{array}$ \\
\hline AY-68-38-106 & 1,196 & 298 & 184 & 114 & drillers' log & TWDB & $7 / 13 / 1973$ & TWDB, 2009 \\
\hline AY-68-38-108 & 1,023 & 405 & 245 & 160 & geophysical log & USGS & $11 / 15 / 1971$ & unpublished data, 1971 \\
\hline AY-68-38-109 & 860 & 287 & 58 & 229 & drillers' log & TWDB & 8/1/1930 & Petitt and George, 1956 \\
\hline AY-68-38-110 & 1,042 & 391 & 279 & 112 & geophysical log & USGS & $8 / 16 / 1973$ & unpublished data, 1973 \\
\hline AY-68-38-111 & 727 & 470 & 314 & 156 & drillers' log & TWDB & $5 / 25 / 1985$ & TWDB, 2009 \\
\hline AY-68-38-1aa & 752 & 233 & 83 & 150 & geophysical log & TWDB & 2/5/1986 & unpublished data, 1986 \\
\hline
\end{tabular}

Publishing support provided by Lafayette Publishing Service Center 
